\title{
Piezoelectricity, Phase Stability, and Surface Diffusion in Multicomponent Nitrides
}

\author{
Christopher Tholander
}

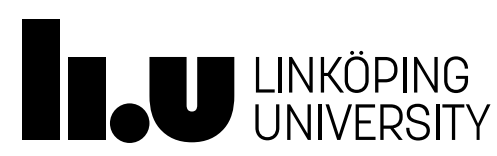

Thin Film Physics Division

Department of Physics, Chemistry, and Biology (IFM)

Linköping University, SE-581 83 Linköping, Sweden 
The cover image is a compilation inspired by key results from my research.

During the course of research underlying this thesis, Christopher Tholander was enrolled in Agora Materiae, a multidiciplinary doctoral program at Linköping University, Sweden.

\footnotetext{
(C) Christopher Tholander

ISBN 978-91-7685-836-3

ISSN 0345-7524
}

Printed by LiU-Tryck 2016 
Till min familj 

The last hundred years have been full of scientific discoveries leading to technological advances, such as, computers, smart phones, etc. Most of the advances would not have been possible without new discoveries within the vast field of materials science. The specific area within materials science covered in this thesis is multicomponent nitride alloys, which are commonly used as thin films in industrial applications, e.g., as hard wear-resistant coatings for cutting-tools or as part of intricate electronic components in mobile telecommunication devices. The core of this thesis is towards the fundamental understanding of existing, and the discovery of new, nitride alloys using theoretical tools. Knowledge about the quantum mechanics of the alloys was gained using density functional theory (DFT), alloy theory, and thermodynamics investigating piezoelectricity, phase stability, and surface diffusion.

The focus of the piezoelectricity research is on piezoelectric properties of both ordered and disordered nitrides. The exploration of disordered wurtzite nitrides revealed important aspects of the nitride alloying physics and the implications for their piezoelectric response, in addition to the discovery of interesting alloy candidates and their synthesis, e.g., $\mathrm{Y}_{x} \operatorname{In}_{1-x} \mathrm{~N}$. For the ordered nitrides, novel $\mathrm{TMZnN}_{2}(\mathrm{TM}=\mathrm{Ti}, \mathrm{Zr}, \mathrm{Hf})$ structures with high piezoelectric responses have been predicted as stable.

The thermodynamic stability of novel alloys with interesting properties is investigated in order to determine if equilibrium or non-equilibrium synthesis is feasible. The studies consist of ternary phase diagrams of TM-Zn-N, mixing enthalpies for disordered $\mathrm{Y}_{x} \mathrm{Al}_{1-x} \mathrm{~N}$ and $\mathrm{Y}_{x} \mathrm{In}_{1-x} \mathrm{~N}$ that can be used to predict possible synthesis routes and guide experiments. In addition, mixing enthalpies for strained $\mathrm{Sc}_{x} \mathrm{Al}_{1-x} \mathrm{~N} / \mathrm{In}_{y} \mathrm{Al}_{1-y} \mathrm{~N}$ superlattices show that the stability of certain phases and, therefore, the crystalline quality can be improved by modifying in-plane lattice parameters through higher indium content in the InAlN layers.

Surface diffusion is studied because it is an important factor during thin film growth with, for example, physical vapor deposition (PVD). It is the main atomic 
transport mechanism and, thus, governs the structure development of thin films. Specifically, the research is focused on diffusion on the surfaces of disordered alloys, and in particular $\mathrm{Ti}, \mathrm{Al}$, and $\mathrm{N}$ adatom diffusion on TiN and TiAlN surfaces. The investigations revealed that $\mathrm{Ti}$ adatom mobilities are dramatically reduced in the presence of $\mathrm{Al}$ in the surface layer on the $\mathrm{TiN}$ and $\mathrm{Ti}_{0.5} \mathrm{Al}_{0.5} \mathrm{~N}(001)$ surfaces, while $\mathrm{Al}$ adatoms are largely unaffected. Furthermore, the reverse effect is found on the $\mathrm{TiN}(111)$ surface, $\mathrm{Al}$ adatom migration is reduced while Ti adatom migration is unaffected. In addition, it is shown that neglecting the magnetic spin polarization of $\mathrm{Ti}$ adatoms will locally underestimate the binding energies and the diffusion

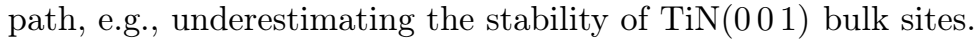




\section{Populärvetenskaplig sammanfattning}

De senast hundra åren har varit fulla av teknologiska framgångar, datorn till exempel har utvecklas från att vara stor som ett hus till att rymmas i en handhållen telefon. Utan materialforskning hade vi inte kunnat komma så långt som vi har i dag, inte heller skulle vi komma mycket längre. Materialforskning är alltså en fundamental del för utvecklingen av våra samhällen. Det är också ett enormt fält med många delområden. Den del av materialforskningen som är fokus för den här avhandlingen är tunna filmer av flerkomponentsnitrider, kväveföreningar innehållande flera olika metaller. Dessa har många industriella användningsområden, så som hårda slitstarka beläggningar för skärverktyg eller intrikata elektroniska komponenter för mobil telekommunikation. Forskningen som utgör den här avhandlingen är inriktad mot att uppnå en grundläggande förståelse av kända nitrider samt att upptäckanda nya. Jag har använt mig av teoretiska verktyg, så som täthetsfunktional-teori som gör det möjligt att undersöka material utifrån hur atomerna och elektronerna i materialet binder till varandra.

För elektroniska komponenter som till exempel används för trådlös överföring eller mobil telekommunikation används piezoelektriska material för att omvandla mekaniskt arbete,vibrationer, till elektriska impulser och vise versa. För att komponenterna ska kunna göras så små som möjligt samt ha ett stort frekvensomfång krävs material med stark piezoelektriskt svar. Arbetet i den här avhandlingen har avslöjat viktiga aspekter som spelar stor roll när oordnade nitrider blandas för att kunna få så starkt piezoelektriskt svar som möjligt. Även nya ordnade titanzinknitrid, zirkoniumzinknitrid, och hafniumzinknitrid strukturer har upptäckts visa starkt piezoelektriskt svar.

Inte alla material som vi upptäcker i teoretiska studier går att tillverka alls eller kan bara tillverkas med specifika metoder. För att kunna avgöra om de material som vi upptäckt kan tillverkas undersöker vi deras fasstabilitet. I det här arbetet har jag sökt och förutspått stabila faser med kombinationer av titan-zink-kväve, zikonium-zink-kväve, eller hafnium-zink-kväve. Jag har även undersökt yttriumaluminiumnitrid och yttriumindiumnitrid i wurtzit struktur som vi även tillverkat 
experimentellt. Utöver detta har jag också undersökt fasstabiliteten hos multilager som består av alternerande skandiumaluminiumnitrid och indiumaluminiumnitrid lager för att avgöra hur kristallkvalitén hos skandiumaluminiumnitrid kan optimeras genom att styra mängden indium i indiumaluminiumnitridlagren.

Under tillväxt av flerkomponentsnitrider är rörelsen av atomer på ytan, så kallad ytdiffusion, en av de starkaste faktorerna som styr hur materialet utvecklas. Jag har studerat hur atomers ytdiffusion påverkas vid legering genom att titta på titan-, aluminium-, och kväveatomer rör sig på titannitrids och titanaluminiumnitrids ytor. I dessa studier har vi upptäckt att de olika atomtyperna påverkas på olika sätt av legering, till exempel skapas fällor för titan atomer på visa ytor när aluminium inkorporeras i ytan. Studien avslöjade även att det är viktigt att ta hänsyn till elektronernas spinn då det kan lokalt påverka bindningen av vissa atomslag, vilket kan ha betydande effekter för dess rörlighet. 
This thesis is a result of my research during my Ph.D. studies in the Thin Film Physics Division at Linköping University from 2011 to 2016. The introduction and methodology parts of this thesis are largely based on my previous Licentiate thesis from 2014, A Theoretical Study of Piezoelectricity, Phase Stability, and Surface Diffusion in Disordered Multicomponent Nitrides, Thesis No. 1675.

My work has been focused on theoretical investigations of piezoelectric properties, thermodynamics, and surface diffusion of multicomponent nitrides. My results have been published in peers reviewed journals, with the exception of Paper VI, which currently is in manuscript-stage, and are appended to this thesis.

Support for this research was received in liaison with Prof. Lars Hultman's Knut and Alice Wallenberg Scholar Grant.

The theoretical calculations have been carried out using supercomputer resources provided by the Swedish national infrastructure for computing (SNIC) at the National Supercomputer Center (NSC), and resources based in Sweden at the Center for High-Performance Computing (PDC) at the Royal Institute of Technology $(\mathrm{KTH})$. 



\section{Acknowledgements}

First of all, I would like to thank my main supervisor Dr. Björn Alling for all of the support during my Ph.D. studies. I've learned a tremendous amount from you.

I would also like to thank my assistant supervisors Prof. Lars Hultman and Dr. Ferenc Tasnádi. Your contributions have been invaluable.

I am also grateful to Prof. Igor Abrikosov who introduced me to theoretical physics by giving me my first project when I was a Masters student.

An extra amount of thanks to all my coauthors who I have had the pleasure working with and learned so much from. Especially Dr. Agnė Žukauskaitè, our theoretical and experimental collaborations have proved to be exceptionally fruitful.

A special thanks goes to the lunch group, for providing friendly company and lunch conversations which have been entertaining and, at times, enlightening.

Awesome thanks also to Jonas Saarimäki, for helping me reach new heights, and being a great friend.

My most important thanks goes to my family, especially Malin, who have always been supportive. I would never have gotten this far without you. I'm immensely lucky and happy to have all of you in my life.

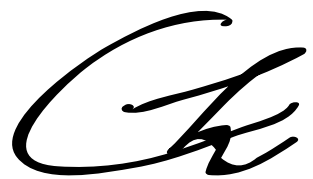

Christopher Tholander Linköping, February 2016 

1 Introduction $\quad \mathbf{1}$

1.1 Theoretical materials science . . . . . . . . . . . . . . . . 2

1.2 Piezoelectric materials . . . . . . . . . . . . . . . . . . 3

1.3 A thin film approach . . . . . . . . . . . . . . . . . . 3

1.4 An atomic scale view of thin film growth . . . . . . . . . . . . . 4

1.5 The aims of my research . . . . . . . . . . . . . 5

2 Methods for modeling atomic and electronic structure $\quad 7$

2.1 Quantum mechanics .................. . . 7

2.2 Density functional theory $(\mathrm{DFT}) \ldots \ldots \ldots$. . . . . . . . . . . . . 8

2.2.1 The Hohenberg-Kohn theorems . . . . . . . . . . . . 9

2.2.2 The Kohn-Sham total energy functional . . . . . . . . . . 9

2.2.3 Local-density approximation (LDA) . . . . . . . . . . . 10

2.2.4 Generalized gradient approximation (GGA) . . . . . . . . 11

2.2.5 Beyond LDA and GGA exchange-correlation functionals . . 11

2.2.6 Choosing basis sets for solving the Kohn-Sham equations . 12

2.3 Modeling random alloys ..................... 13

2.3.1 Configurational disorder in alloys . . . . . . . . . . . 13

2.3.2 The special quasi-random structure (SQS) model . . . . . . 14

2.3.3 Limitations of the SQS model . . . . . . . . . . . 15

3 Piezoelectric nitrides $\quad 17$

3.1 Elastic properties . . . . . . . . . . . . . . 17

3.1.1 Elastic constants in disordered structures . . . . . . . . . 19

3.2 Berry-phase theory of polarization . . . . . . . . . . . . . 20

3.3 Piezoelectric properties . . . . . . . . . . . . . . 20

3.4 Increasing the piezoelectric response of AlN . . . . . . . . . . . . 23

3.5 Expanding the search for piezoelectric ternary nitrides . . . . . . . 24

3.5.1 Improvement of the piezoelectric response . . . . . . . . . 25 
3.5.2 A volume matching condition . . . . . . . . . . . 27

3.5.3 $\mathrm{Y}_{1-x} \operatorname{In}_{x} \mathrm{~N} \ldots \ldots \ldots \ldots 27$

3.6 Expanding the search to quarternary alloys . . . . . . . . . . . 29

3.6.1 Improvement of the piezoelectric response . . . . . . . . . . 30

3.6.2 Descriptors for good alloying components for AlN . . . . . 30

$3.7 \mathrm{TM}_{0.5} \mathrm{Zn}_{0.5} \mathrm{~N}$ alloys . . . . . . . . . . . . . . . . . . . 32

4 Thermodynamics of materials $\quad 33$

4.1 Gibb's free energy . . . . . . . . . . . . . . . . . 33

4.1.1 Approximating Gibb's free energy for non-equilibrium conditions . . . . . . . . . . . . . . . 35

4.2 Phase stability of multicomponent alloys . . . . . . . . . . . . . 36

4.3 Phase stability of $\mathrm{TM}_{0.5} \mathrm{Zn}_{0.5} \mathrm{~N}$ alloys . . . . . . . . . . . . 37

5 Thin film deposition 41

5.1 Sputtering ........................ 41

5.2 Magnetron setup . . . . . . . . . . . . . . . . . . 42

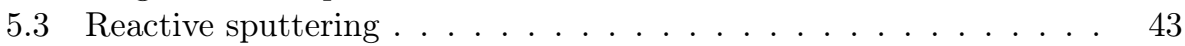

5.4 Substrate setup . . . . . . . . . . . . . . . . . . . . . . . . . . . . . . . 43

5.5 Sputtering multiple materials . . . . . . . . . . . . . . . . 43

5.6 Implications . . . . . . . . . . . . . . . . . . . . . . . . . . 44

6 Metastable nitride alloys $\quad \mathbf{4 5}$

6.1 Ternary nitride alloys . . . . . . . . . . . . . . . . . 45

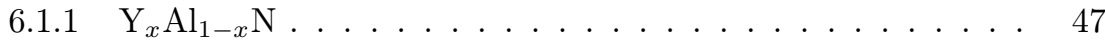

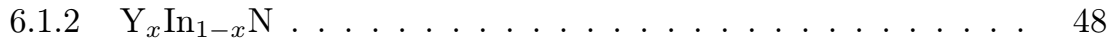

6.2 Quarternary nitride alloys . . . . . . . . . . . . . . . 49

6.2.1 $\mathrm{TM}_{x / 2} \mathrm{Mg}_{x / 2} \mathrm{Al}_{1-x} \mathrm{~N}$ and $\mathrm{TM}_{x / 2} \mathrm{Ca}_{x / 2} \mathrm{Al}_{1-x} \mathrm{~N} \quad(\mathrm{TM}=\mathrm{Ti}$, $\mathrm{Zr}, \mathrm{Hf}) \ldots \ldots \ldots \ldots . \ldots \ldots$

6.2.2 $\mathrm{TM}_{x / 2} \mathrm{Zn}_{x / 2} \mathrm{Al}_{1-x} \mathrm{~N}(\mathrm{TM}=\mathrm{Ti}, \mathrm{Zr}, \mathrm{Hf}) \ldots \ldots . . . . . \quad 51$

6.3 Strained systems ..................... 51

7 Kinetics in disordered alloys $\quad \mathbf{5 5}$

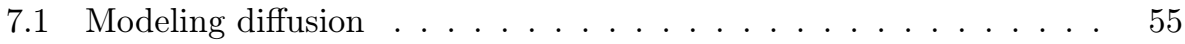

7.1 .1 Fick's first law . . . . . . . . . . . . . . . 57

7.1 .2 Fick's second law . . . . . . . . . . . . . . . 57

7.1.3 Generalizing Fick's laws to include additional dimensions . 59

7.1.4 Diffusion temperature dependence . . . . . . . . . . . 59

7.1.5 Calculating the diffusion activation barrier . . . . . . . . 60

7.1.6 Approximating the diffusion prefactor . . . . . . . . . 62

7.1.7 Diffusivity on surfaces with multiple binding site types . . . 63

7.2 Hard coatings . . . . . . . . . . . . . . . . . 67

7.3 Disordered $\mathrm{Ti}_{1-x} \mathrm{Al}_{x} \mathrm{~N}$ surface diffusion . . . . . . . . . . . . . . 68

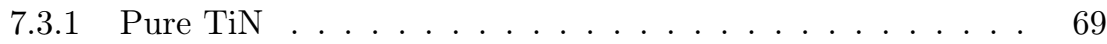

7.3.2 Disordered $\mathrm{Ti}_{0.5} \mathrm{Al}_{0.5} \mathrm{~N} \ldots \ldots \ldots \ldots$. . . . . . . . . . . . . . 69

7.3.3 Effect of single Al substitution . . . . . . . . . . . . 71

7.3.4 Spin polarization effects on adatom diffusion . . . . . . . 74 
8 Conclusions $\quad \mathbf{7 7}$

8.1 Piezoelectric nitrides . . . . . . . . . . . . . . . . 77

8.2 Phase stability . . . . . . . . . . . . . . . . . . . . . 78

8.3 Surface Diffusion . . . . . . . . . . . . . . . . . 79

9 Outlook $\quad \mathbf{8 1}$

9.1 Piezoelectric nitrides . . . . . . . . . . . . . . . . . 81

9.2 Phase stability . . . . . . . . . . . . . . . . . . 81

9.3 Surface diffusion . . . . . . . . . . . . . . . 82

$\begin{array}{lr}\text { Bibliography } & 83\end{array}$

$\begin{array}{ll}\text { List of Publications } & 93\end{array}$

$\begin{array}{ll}\text { Summary of Included Publications } & 95\end{array}$

$\begin{array}{lr}\text { Paper I } & 99\end{array}$

$\begin{array}{ll}\text { Paper II } & 107\end{array}$

$\begin{array}{ll}\text { Paper III } & 115\end{array}$

$\begin{array}{ll}\text { Paper IV } & 125\end{array}$

$\begin{array}{lr}\text { Paper V } & 137\end{array}$

$\begin{array}{ll}\text { Paper VI } & 145\end{array}$

$\begin{array}{ll}\text { Paper VII } & 153\end{array}$

$\begin{array}{ll}\text { Paper VIII } & 163\end{array}$

$\begin{array}{ll}\text { Appendix A Periodic table } & 179\end{array}$

$\begin{array}{lr}\text { Appendix B Glossary and acronyms } & 181\end{array}$

Glossary . . . . . . . . . . . . . . . . . . 181

Acronyms ......................... 183 


\section{CHAPTER 1}

\section{Introduction}

Materials have been a key part in the history of mankind, to such extent that historians and archeologists have seen it fit to divide our history into ages named after the prominent material used or introduced during that age.

The first materials age, the stone age, began more than 2.5 million years ago. However, the first stone tools appeared as early as 3.36 million years ago [1], even before the advent of the Homo genus. The age is divided into three phases, Paleolithic (Old stone age), Mesolithic (Middle stone age), and Neolithic (New stone age) [2]. Stone, e.g., flint, is a very hard material but it is also brittle, meaning it will break without significant deformation. Therefore, striking flint with another stone causes flakes to detach from it, not just denting it, creating sharp edges that are excellent for cutting tools. Also metals, such as copper, silver and gold, was used during the neolithic phase. These can be worked in to many forms because it can be soften by heating and hardened by hammering. Because the metals were rare and soft compared to stone, tools were still made of stone and metals were mainly used to create ornaments or decorations.

The first of the metal ages is the bronze age, where the first phase is the Chalcolithic (Copper-Stone age). Copper use becomes increasingly important during this period, in part because copper was the most abundant of the accessible metals. However, the pure copper is not hard enough and ways to improve it was needed. This was done by alloying copper with other metals, some less successful than others. For example, copper can be alloyed with arsenic to improve its hardness, but the fumes from the process are deadly. Eventually, the combination copper and tin (bronze) was found to be the most successful, being harder and less brittles than pure copper. Of course even harder, more durable, and more abundant materials were sought for.

The next age was the iron age, which started in different parts of the world around 1500 to 1000 B.C [2]. Because iron has a high melting temperature it was 
initially obtained as a slag product, called bloom, from copper smelting operations. Iron in this form is porous, and workable at much lower temperatures. Moreover, pure iron is not harder than bronze so it had to be alloyed with an other material before there was an advantage to use it. The most successful alloys incorporated varying amounts of carbon, creating different types of steel.

The search for new materials so far had been empirical, and many believed magic was part of the process. Not until the 19th and 20th century, was there a systematic approach to the search for new materials to a degree which could be described as a material science. This qualitative step could be taken, to a large part, because we began to understand materials at an atomic level, discovering the fundamentals of chemistry and the proton, neutron, and electron. This was also when electronic materials emerged to a greater extent, becoming a pivotal part in 20th and 21st century materials science.

\subsection{Theoretical materials science}

Finding new and improved materials is no simple task. If we allow there to be any number of components at any concentrations in our material, then there is an infinite amount of ways to combine the elements of the periodic table into different alloys. Making and testing the properties of even a small fraction of these materials would certainly take an unbearably long time and require vast resources. Although there are ways to reduce the number of potential combinations using carefully designed experimental series, the amount of possibilities are still numerous. Therefore, it is important to understand how materials work at an atomic and electronic level, and let that knowledge guide our search for better materials. The accumulation and systematization of this understanding is the theoretical part of materials science, which during the last couple of decades has been accelerated enormously using computer simulations.

Given that the theoretical framework is accurate enough, there are plenty of advantages with computer simulations compared to experiments. It is possible to run simulations with toxic or radioactive materials without posing a health risk, isolate specific physical effects in controlled environments, and work in parallell with multiple materials. It is also possible to test expensive materials without any added cost. Of course, there are also limitations to computer simulations. There is usually a trade off between wether a simulation should be accurate (e.g. include quantum effects) or if it should be large (e.g. include many atoms or long time scales). Often the choice between the two depends on the problem that is investigated, e.g., some problems require long time scales and thousands of atoms which means the accuracy needs to be reduced. However, computers improve at an amazing rate, making them faster and able to handle more complex problems. Thus, we are continuously able to push the boundaries of what we can simulate even further. Continued development of the accuracy of the theoretical methods goes hand in hand with computations of specific materials research topics. This work is a piece in that big puzzle, by extending the frontiers of multicomponent nitrides, discovering novel piezoelectric materials, and studying growth related 
features, i.e., phase stability and surface diffusion.

\subsection{Piezoelectric materials}

Piezoelectric materials are a class of materials that is important in a wide range of microelectromechanical systems (MEMSs), e.g., sensors, actuators, and energy harvesters that can power micro-sized devices, is piezoelectric materials [3-7]. These materials can convert vibrations to electrical energy, or the opposite, electrical energy into vibrations.

The reason why a material exhibit a piezoelectric effect is because it changes its polarization upon deformation. Polarization comes from a shift between the positively and negatively charged ion position in the lattice. When mechanical strain is applied in a direction which increases or decreases the shift between the ions, there will be a flow of electrons to counteract the change in polarization. The reverse piezoelectric effect is simply the process in reverse, an applied voltage forces a shift of the ions to counteract the voltage.

There is not one universal piezoelectric material optimal for all applications. By discovering new piezoelectric materials it can be possible to improve current devices, and possibly inspire completely new applications.

\subsection{A thin film approach}

Not all advances in materials science have emerged from improving a material's properties by alloying. One advance of particular interest in this thesis comes from the fairly simple idea that the surface properties of one material can be improved by coating it with another material with superior surface properties, but not necessarily good bulk properties. Thus, it is possible to combine the bulk properties of one material with the surface properties of another. For example, we can coat materials with a thin film to improve its oxidation resistance, wear resistance, or as a purely decorative coating. A coating does not even have to be very thick. What we characterize as a thin film ranges from a single monolayer in thickness to a few micrometers, which is in the order of 10000 atomic layers. Although the cross section of such a film is too thin to be visible with our naked eye, it is enough to greatly improve the surface properties of a bulk material. Also, in this thin film form, due to the particularities of thin film synthesis methods, it is possible to grow materials which are thermodynamically only metastable, and in some cases impossible to create in bulk form. Thus, these materials can be used in practical applications if they exhibit extraordinary properties, e.g., the high hardness of $\mathrm{Ti}_{x} \mathrm{Al}_{1-x} \mathrm{~N}$ [8-10] or the large piezoelectric response in $\mathrm{Sc}_{x} \mathrm{Al}_{1-x} \mathrm{~N}$ [11]. These particular alloys can be synthesized despite having a thermodynamic driving force for phase separation into almost pure binary nitrides at all relevant temperatures $[12,13]$.

To synthesize metastable thin films is no simple task. It is not just a matter of mixing liquid metal and poring it in a mold. Instead, other techniques need to be used, e.g., PVD. This family of techniques allow for synthesis at low and 
intermediate temperatures where the kinetic is limited to such a degree that both diffusion and chemical separation on the metal sub-lattice is quenched. However, if the materials are subjected to heat treatment the diffusion is reenabled and decomposition can be observed [14]. These materials are as synthesized stuck in a local minimum of Gibb's free energy, restricted by kinetic or thermodynamic barriers large enough to make their transition to their global minimum macroscopically slow.

Another thin film synthesis technique is chemical vapor deposition (CVD). With this technique, films are grown by chemical reactions between gas precursors that then adhere to a substate. This approach typically requires high temperatures to be effective $\left(\sim 1000{ }^{\circ} \mathrm{C}\right)$. The process is often ideal for coating objects with complex shapes since all surfaces in contact with the gas will be coated. One of the main benefits of this method is that it can be used to coat large areas relatively fast. The main focus in the present work is, however, PVD.

\subsection{An atomic scale view of thin film growth}

The surface diffusion, i.e. movement, of the adatoms and admolecules on a substrate surface, and subsequently on layers of the film, is very important during both PVD and CVD growth because it governs the evolution of the film. If the rate at which atoms are added to the surface is low, then there is time for the adatoms already at the surface to arrange themselves, filling gaps making the film dense and even. In the opposite case, when the rate of arriving atoms is very high, the films will become rough and possibly porous. This is because adatoms might start to form many small islands instead of few large ones, and there will not be enough time to fill in the voids between them before the next layer starts to grow.

There are many aspects that influence adatom surface diffusion other than surface material and adatom species, e.g., surface crystal orientation, and impurities. The adatom movement can differ a lot between surface orientations [15]. This can have an effect on the preferred orientation of the film, promoting one direction over another [16].

During alloy growth, there are multiple adatom species diffusing at the same time. If there is a large variation in, for example, how fast they diffuse on different surface orientations; then the composition could vary between grains, resulting in inhomogeneous films. It is important to understand how different adatoms diffuse, in order to either promote or counter such effects.

Currently much is known about the surface diffusion on elemental metal surfaces. However, very little is known about multicomponent alloys. The challenges in understanding such materials stem, in part, from added complexity from configurational disorder. Also, effects of impurities in alloy surfaces on surface diffusion are mostly unknown. It is likely that they can effect adatom diffusion in many different ways, by promoting or obstructing the diffusion path for various adspecies. 


\subsection{The aims of my research}

The general purpose of the research has been to further the fundamental understanding of piezoelectric response, phase stability, and surface diffusion in nitrides. In addition, suggestions for new materials with interesting properties have been searched for building on this understanding. The materials that I have studied can be or are already applied in thin film application because they are metastable. The research has been focused in three areas: piezoelectricity, thermodynamics, and surface diffusion.

My main focus within piezoelectricity has been to investigate how the piezoelectric response of a nitride compound can be increased by alloying and why, creating ternary or quarternary alloys.

Within thermodynamics I have studied various effects in order to critically evaluate how theory can be used to guide experiments on metastable nitride alloys. This is done by studying the mixing enthalpies, where phase curve crossing points and absolute values are key characteristics.

Finally, within the field of surface diffusion I have explored the effects of alloying on surface diffusion, a principal mechanism during thin film growth and crucial in the development of metastable phases. The aim is to to link static DFT surface energetics to the mobility of adatoms during growth. 


\section{CHAPTER 2}

\section{Methods for modeling atomic and electronic structure}

The main theories behind the tools that are used to model a materials atomic and electronic structure are described in this chapter. The contents cover quantum mechanics, DFT, and configurational disorder.

\subsection{Quantum mechanics}

The main reason for why we are able to do predictions of material properties directly from theory is the high accuracy of quantum mechanics in the description of atomic nuclei and electrons. Within quantum mechanics, we can describe a system of $N$ nuclei and $n$ electrons using a wave function $\Psi$,

$$
\Psi=\Psi\left(\mathbf{r}_{1}, \mathbf{r}_{2}, \ldots, \mathbf{r}_{n}, \sigma_{1}, \sigma_{2}, \ldots, \sigma_{n}, \mathbf{R}_{1}, \mathbf{R}_{2}, \ldots, \mathbf{R}_{N}, t\right)=\Psi(\overline{\mathbf{r}}, \bar{\sigma}, \overline{\mathbf{R}}, t),
$$

which is a function of the positions of the electrons $\mathbf{r}_{i}$, electron spins $\sigma_{i}$, nuclei positions $\mathbf{R}_{I}$, and time. The wave function is the solution to the Schrödinger equation, which determine the time evolution of the system. In atomic units the Schrödinger equation is

$$
i \frac{\partial \Psi}{\partial t}=H \Psi
$$

where $H$ is the Hamiltonian. In a system of nuclei and electrons without an external potential, the Hamiltonian is

$$
\begin{aligned}
H=-\frac{1}{2} \sum_{i=1}^{n} \nabla_{i}^{2}-\frac{1}{2} \sum_{I=1}^{N} \frac{1}{M_{I}} \nabla_{I}^{2}- & \sum_{i, I} \frac{Z_{I}}{\left|\mathbf{r}_{i}-\mathbf{R}_{I}\right|} \\
& +\frac{1}{2} \sum_{i \neq j} \frac{1}{\left|\mathbf{r}_{i}-\mathbf{r}_{j}\right|}+\frac{1}{2} \sum_{I \neq J} \frac{Z_{I} Z_{J}}{\left|\mathbf{R}_{I}-\mathbf{R}_{J}\right|} .
\end{aligned}
$$


The terms in the Hamiltonian correspond to, from left to right, the kinetic energy of the electrons, the kinetic energy of the nuclei, the potential energy of the electron-nucleus interaction, the electron-electron interaction, and the nucleusnucleus interaction.

If there is no specific time dependence in the Hamiltonian, it is possible to separate Eq. (2.2) in a time dependent and a spatial part. In many cases it is sufficient to look at the time independent part of the Schrödinger equation,

$$
H \Phi(\overline{\mathbf{r}}, \bar{\sigma}, \overline{\mathbf{R}})=E \Phi(\overline{\mathbf{r}}, \bar{\sigma}, \overline{\mathbf{R}})
$$

where $\Phi(\overline{\mathbf{r}}, \bar{\sigma}, \overline{\mathbf{R}})$ is the eigenfunction solution with the corresponding total energy eigenvalue $E$. Although Eq. (2.4) looks simple, solving it for systems with more than one hydrogen atom (two particles) quickly increases the difficulty.

It is worth to point out that we actually do not solve the Schrödinger equation in the codes we use to perform the calculations. Instead, a scalar-relativistic version of the Dirac equation is used. The fundamental points are, however, not different from when using the Schrödinger equation and the notation also become less cumbersome.

The Born-Oppenheimer approximation [17] is a very useful first approximation to reduce the number of particles dealt with in each calculation step. This is possible because the mass of the nuclei and the electrons differ by several orders of magnitude. Therefore, it is most of the times possible to deal first with electrons in a constant potential field caused by the nuclei. The kinetic effects on the nuclei can then be dealt with in a second step, in which the electrons are considered to remain in their ground state.

The problem with too many variables can be simplified in bulk crystalline materials with a second useful simplification, available because of the Bloch-theorem [18]. This allows us to use the periodicity of the crystal, and calculate only the unit cell of the crystal structure instead of every electron and nucleus in a macroscopic crystal. The wave function solutions to the Shrödinger equation then takes the form

$$
\phi_{n \mathbf{k}}(\overline{\mathbf{r}})=e^{i \mathbf{k} \cdot \mathbf{r}} u_{n \mathbf{k}}(\overline{\mathbf{r}})
$$

where $n$ is the quantum number, $\mathbf{k}$ is a reciprocal vector, $u_{n \mathbf{k}}(\overline{\mathbf{r}})$ is a function with the periodicity of the lattice, and $e^{i \mathbf{k} \cdot \mathbf{r}}$ describes a plane wave. Although the problem at this point has been greatly simplified, there is so far no loss in accuracy. However, solving a system with more than a few electrons by solving the Schrödinger equation directly still poses a near impossible challenge. At this point, something more is needed to be done. This is where DFT comes in.

\subsection{Density functional theory (DFT)}

The basic idea of DFT is to use the electron density $n(r)$ as the basic variable instead of the wave function. This simplifies the problem of working with $3 n$ spatial variables for a $n$-electron problem to a problem of only three. The first attempts to use density functionals was in 1927 with the Thomas-Fermi theory 
$[19,20]$. However, the theory could not reproduce bonding between atoms, so it was generally discarded as a non-practical approach for many years.

\subsubsection{The Hohenberg-Kohn theorems}

It took until 1964, when Hohenberg \& Kohn published their paper on inhomogeneous electron gas [21], for DFT to emerge in its modern form. The two theorems are stated in the words of Martin [22] as follows:

Theorem 1 For any system of interacting particles in an external potential $V_{\text {ext }}(\mathbf{r})$, the potential $V_{\text {ext }}(\mathbf{r})$ is determined uniquely, except for a constant, by the ground state particle density $n_{0}(\mathbf{r})$.

Theorem 2 A universal functional for the energy $E[n]$ in terms of the density $n(\mathbf{r})$ can be defined, valid for any external potential $V_{\text {ext }}(\mathbf{r})$. For any particular $V_{\text {ext }}(\mathbf{r})$, the exact ground state energy of the system is the global minimum value of this functional, and the density $n(\mathbf{r})$ that minimizes the functional is the exact ground state density $n_{0}(\mathbf{r})$.

The first theorem has the consequence that all properties of a system are completely determined by the ground state density $n_{0}(\mathbf{r})$, and the second that $E[n]$ alone is enough to determine the exact ground state energy and density. The general form of the functional is

$$
E[n]=T[n]+E_{\text {int }}[n]+\int d^{3} r V_{e x t}(\mathbf{r}) n(\mathbf{r})+E_{I I}
$$

where the terms on the right side of the equation represent the kinetic energy of the electrons, the electron-electron interaction energy, the interaction energy with an external potential in the form of Coulomb interaction with the nuclei, and the interaction energy between the nuclei. Although a practical scheme to solve the equations was not presented at this point, it was proposed already the year after the Hohenberg-Kohn theorems by Kohn \& Sham.

\subsubsection{The Kohn-Sham total energy functional}

Kohn \& Sham suggested in 1965 [23] that the real system with interacting particles should be replaced with a system of noninteracting particles, in which the ground state density is assumed to be equal to that of the original interacting system. This is done by replacing the purely external potential with an effective potential $V_{\text {eff }}$. The single particles interacting with the effective potential is then described by the single particle wave function $\psi_{j}$, which is found by solving the single-particle equation [24]

$$
\left(-\frac{1}{2} \nabla^{2}+V_{e f f}(\mathbf{r})-\epsilon_{j}\right) \psi_{j}(\mathbf{r})=0,
$$


where $\epsilon_{j}$ is the eigenvalue of the non-interacting single particle, and the effective potential is given by

$$
V_{e f f}(\mathbf{r})=V_{e x t}(\mathbf{r})+\int \frac{n\left(\mathbf{r}^{\prime}\right)}{\left|\mathbf{r}-\mathbf{r}^{\prime}\right|} d \mathbf{r}^{\prime}+V_{x c}(\mathbf{r}),
$$

where

$$
V_{x c}=\frac{\delta E_{x c}[n(\mathbf{r})]}{\delta n(\mathbf{r})} .
$$

The electron density in a system with $N$ electrons is then calculated using the wave functions according to

$$
n(\mathbf{r})=\sum_{j=1}^{N}\left|\psi_{j}(\mathbf{r})\right|^{2} .
$$

The total energy is given by the Kohn-Sham total energy functional

$$
E_{K S}[n]=T_{s}[n]+\int d \mathbf{r} V_{e x t}(\mathbf{r}) n(\mathbf{r})+E_{\text {Hartree }}[n]+E_{I I}+E_{x c}[n] .
$$

Here, the $T_{s}$ is the independent-particle kinetic energy given by

$$
T_{s}=-\frac{1}{2} \sum_{i=1}^{N}\left\langle\psi_{i}\left|\nabla^{2}\right| \psi_{i}\right\rangle,
$$

$V_{\text {ext }}(\mathbf{r})$ is the external potential due to the nuclei and any other external fields (assumed to be independent of spin), $E_{I I}$ is the interaction between the nuclei, and $E_{\text {Hartree }}$ is the classical Coulomb interaction energy of the electron density with itself given by

$$
E_{\text {Hartree }}=\frac{1}{2} \int d^{3} r d^{3} r^{\prime} \frac{n(\mathbf{r}) n\left(\mathbf{r}^{\prime}\right)}{\left|\mathbf{r}-\mathbf{r}^{\prime}\right|} .
$$

Although the Kohn-Sham total energy functional is exact in the form presented in Eq. (2.11), the exchange-correlation term $E_{x c}[n]$, where all many-body effects are included, pose a problem since there is no universal form for it. However, effects of this term is in general small compared to the other terms which are evaluated almost exactly. There also exist approximate functionals which capture manybody effects with good accuracy, e.g., the local-density approximation (LDA) and the generalized gradient approximation (GGA) which are presented below.

\subsubsection{Local-density approximation (LDA)}

LDA was first suggested in the original paper by Kohn \& Sham [23], and later extended to also include spin with the local spin-density approximation (LSDA) [25]. The basic idea comes from the observation that exchange-correlation effects are to a large extent local in character. Therefore, they proposed to calculate the exchange-correlation energy $E_{x c}^{L D A}[n]$ with a simple integral over all space, 
where the exchange-correlation energy density is assumed to be the same as in homogeneous electron gas with that density $\epsilon_{x c}^{\text {hom }}[n(\mathbf{r})]$,

$$
E_{x c}^{L D A}[n]=\int d^{3} n(\mathbf{r}) \epsilon_{x c}^{h o m}[n(\mathbf{r})] .
$$

The homogeneous electron gas has been studied to great accuracy using quantum Monte Carlo (QMC) calculations [26], creating a set of data for different densities. The densities between the points in the data set has then been interpolated $[27,28]$.

The initial expectations about the performance of LDA was that it would only work well in the limit where the density varies slowly. However, LDA turned out to produce useful results also for more complex systems [24]. This is because of the behavior of the exchange-correlation hole, the many-body effect of depleting the charge of other electrons closest to each electron. LDA obeys the sum rule, that if we integrate the entire exchange-correlation hole we will end up with exactly the charge of one electron, even if the shape of the hole is not entirely correct. The reason why the shape of the hole does not matter that much is because only the spherical and system average of the exchange-correlation hole enters the energy, and this is well reproduced by LDA.

To summarize the performance of LDA, it works well for covalent-, metalic-, and ionic-type bonds, even though it generally tends to overbind slightly. Although the overbinding causes gives lattice parameters which are lower than those experimentally obtained, trends in lattice parameters are well reproduced. However, for long range interactions, such as Van der Waal's interactions, LDA does not work.

\subsubsection{Generalized gradient approximation (GGA)}

GGA improves on LDA by including the absolute value of the gradient of the density at each point. There are a number of implementations to introduce the gradient corrections [29-32], which still force the system to behave correctly in important limiting cases [22].

The work in this thesis has been performed mostly using the Perdew, Burke, and Ernzerhof GGA (PBE-GGA) implementation [31, 33]. Although it tends to underbind and provide slightly larger lattice constants than those obtained experimentally, PBE-GGA is better at providing reasonable geometries for the nitride systems investigated in this thesis, as compared to LDA.

\subsubsection{Beyond LDA and GGA exchange-correlation function- als}

The limitations in LDA and GGA has called for many new functionals, e.g., $\mathrm{LDA}+\mathrm{U}[34-36]$ and hybrid functionals such as B3LYP [29, 37], improving features such as bandgaps and long range interactions.

Another issue is the accuracy of adsorption and surface energies obtained by GGA has received some criticism lately by Schimka et al. [38], pointing out limitations in commonly used pseudopotentials. The main problem they address is 
for configurations where van der Waals forces are important contributors, such as admolecules. However, there is no evidence this would be an alarming issue for single adatoms. For a single adatom, the van der Waals force contribution to the adsorption energy is much less than the short range binding to the nearest surface atoms of more covalent or ionic-like character.

A general issue with most post LDA and GGA exchange-correlation methods is that the increased accuracy also greatly increases the computational time, usually many times more the LDA and GGA computational time. Therefore, using one of these improved functionals for problems where LDA and GGA anyway performs well is not economical in terms of computer resources.

\subsubsection{Choosing basis sets for solving the Kohn-Sham equa- tions}

Basis sets are used when solving the Kohn-Sham equations numerically, there they are used to expand the wave function in Eq. (2.1). A natural choice is to use plane waves as the basis set, because they work well with tools related to Fourier transforms. However, the rapid variations in the wave functions and effective potentials close to the nuclei are problematic to describe with plane waves. A huge set of plane waves with high energy cutoff would be needed in order to simultaneously describe the environment close to the nuclei with high kinetic energies as well as the smother region between the atoms. Using pseudopotentials is one way to get around this problem.

The basic idea of the pseudopotential is to replace the problematic inner region of the atom, the nucleus and the inner core electrons, with an effective ionic potential acting on the valence electrons. With this approach, the basis set size and the number of electrons are reduced. Thus, solving the problem is faster.

Although there are many different kinds of pseudopotentials which can be used to simplify the calculations, the general approach is to construct the potential in a way that it reproduces the scattering properties of the core region within a certain cutoff radius and the behavior of the valence wave functions and the effective potentials outside it.

Soft pseudopotentials employ a larger cutoff radius, this does provide faster converging calculations, however, it also makes them less transferable. Not being able to transfer a pseudopotential between crystal structure or chemical environments means that one has to go through the extensive work of parameterize a new one for each new material or crystal structure. An approach to solve this problem with transferability is to use ultrasoft pseudopotentials [39], which was used in the calculations in Paper I. In this approach, this is accomplished by introducing a generalized orthonormality condition, and making sure that the full electronic charge is recovered, by augmenting the electron density in the core regions.

The majority of the calculations presented in the papers were performed using projector augmented wave (PAW) approach [40, 41]. Although the PAW method is similar to the ultrasoft approach, it retains the entire set of all-electron core functions along with the smooth parts of the valence functions. This makes it possible to reconstruct all electron wave functions from the pseudo-wavefunctions. 
The two approaches are similar in accuracy, however, the PAW is more reliable for magnetic systems [41].

\subsection{Modeling random alloys}

A review on the subject theoretical modeling of random alloys is covered in Ref. [42]. The following section is inspired by that work.

\subsubsection{Configurational disorder in alloys}

One of the most challenging problems with modeling alloys like $\mathrm{Ti}_{1-x} \mathrm{Al}_{x} \mathrm{~N}$ is the configurational disorder, where the $\mathrm{Ti}$ and $\mathrm{Al}$ atoms are positioned more or less randomly at cation sites in the lattice. In random alloys, there is no longrange order, although short-range order can exist, therefore, the Blochs theorem (Sec. 2) is no longer valid. Thus, the crystal unit cell is not enough to simulate the material's properties. In practice, this can be modeled with a supercell of several unit cells, but the larger the system, the more computer resources are required. Therefore, we want to use an as small simulation box as possible which still represent the properties of the random alloy. In a formal sense, a random alloy can be defined as a system with an atomic configuration in the limit $V / T \rightarrow 0$, where $V$ is the strongest effective configurational interaction in the system and $T$ is the temperature.

The importance of using a random configuration is illustrated in Fig. 2.1, where (a) and (b) are cells representing two possible outcome of randomly generated configurations of colored and white atoms. In this example it might appear intuitive which one would best represent a random alloy when periodic boundaries are applied, whereas the situation is actually very complex. Should we continue to randomly place atoms and by increasing the size too infinity, then it would practically not matter which supercell is used. Of course, an extremely large supercell

(a)

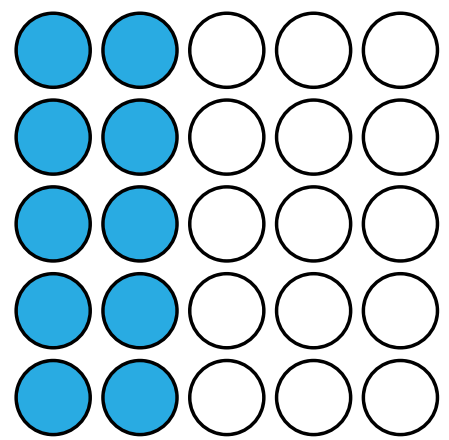

(b)

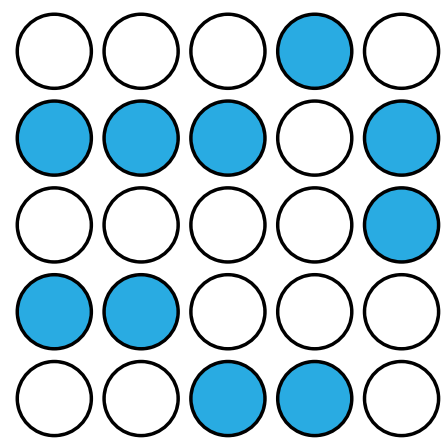

Figure 2.1. Simple illustration of the configurational problem. System (a) and system (b) has the same composition, but different configurations of atoms. 
is not useful from a calculation point of view. Instead, it is advisable to use the special quasi-random structure models [43].

\subsubsection{The special quasi-random structure (SQS) model}

One way to model random alloys is to use special quasi-random structure (SQS) models, generated using the approach first suggested by Zunger et al. [43]. To understand this method it is necessary to understand the concept of cluster expansion of the configurational part of the total energy. The formalism necessary for this was developed by Sanchez et al. [44, 45].

For a simple case of a binary alloy, $\mathrm{A}_{x} \mathrm{~B}_{1-x}$, we can use spin variables $\sigma_{i}$ to describe the atomic configuration. $\sigma_{i}$ takes on the value +1 if the site is occupied by an $\mathrm{A}$ atom and -1 if its occupied by a $\mathrm{B}$ atom. In a crystal with $N$ sites, the vector containing all the spin variables is then $\boldsymbol{\sigma}=\left\{\sigma_{1}, \sigma_{2}, \sigma_{3}, \ldots, \sigma_{N}\right\}$. A characteristic function $\Phi_{f}^{(n)}(\boldsymbol{\sigma})$ can be defined for a given $n$-site cluster, which is given by the product of the spin variables $\sigma_{i}$ in the cluster $\alpha$,

$$
\Phi_{\alpha}^{(n)}(\boldsymbol{\sigma})=\prod_{i \in \alpha} \sigma_{i}
$$

These functions form a complete and orthogonal set with the inner product

$$
\left\langle\Phi_{\alpha}^{(n)}(\boldsymbol{\sigma}), \Phi_{\beta}^{(n)}(\boldsymbol{\sigma})\right\rangle=\frac{1}{2^{N}} \sum_{\boldsymbol{\sigma}} \Phi_{\alpha}^{(n)}(\boldsymbol{\sigma}) \Phi_{\beta}^{(n)}(\boldsymbol{\sigma})=\delta_{\alpha, \beta},
$$

where the summation goes over all possible configurations $\boldsymbol{\sigma}$. This means that if two clusters differ by at least one site, then the function equals to 0 , and 1 if they are the same. This means that any function of the configuration

$$
F(\boldsymbol{\sigma})=\sum_{\alpha} F_{\alpha}^{(n)} \Phi_{\alpha}^{(n)}(\boldsymbol{\sigma})
$$

can be expanded in this basis set. The expansion coefficient in this equation are purely the projections

$$
F_{\alpha}^{(n)}=\left\langle F(\boldsymbol{\sigma}), \Phi_{\alpha}^{(n)}(\boldsymbol{\sigma})\right\rangle
$$

on the basis function.

In the case of total energy $E_{t o t}$ of an alloy configuration, the expansion coefficients are called effective cluster interactions $V_{\alpha}^{(n)}$, given by

$$
V_{\alpha}^{(n)}=\left\langle E_{t o t}(\boldsymbol{\sigma}), \Phi_{\alpha}^{(n)}(\boldsymbol{\sigma})\right\rangle
$$

If we introduce the definition of the statistical cluster correlation function $\xi_{f}^{(n)}$ for a given configuration $\boldsymbol{\sigma}$ as the average of the symmetrically identical cluster functions,

$$
\xi_{f}^{(n)}(\boldsymbol{\sigma})=\left\langle\Phi_{f}^{(n)}\right\rangle=\frac{1}{m_{f}^{(n)}} \sum_{\forall \alpha \in f} \Phi_{\alpha}(\boldsymbol{\sigma})
$$


where $m_{f}^{(n)}$ is a normalization factor. The total energy can then be given in terms of symmetrically non-equivalent figures ${ }^{1}$ using the expression

$$
E_{t o t}=\sum_{f} V_{f}^{(n)} m_{f}^{(n)} \xi_{f}^{(n)} .
$$

From Eq. (2.21), it is possible to draw conclusions about how a supercell should be constructed in order to mimic a truly random structure. First of all, it is clear that the only clusters that can contribute to the alloy energetics are the ones where $V_{f}^{(n)} \neq 0$. This means that only their correlation functions are important. Therefore, an SQS supercell should be constructed so that $\xi_{f}^{(n)}=0$, as it is in a random structure, for as many of these clusters as possible. ${ }^{2}$ Although a finite supercell will not be able to do this for all distance cluster, the interactions corresponding to pair clusters at short distances are generally more important than those between more distant neighbors. Therefore, an SQS will best represent the random alloy if it is generated with as many of the first few nearest neighbor correlation functions as possible equal to zero.

\subsubsection{Limitations of the SQS model}

Although the SQS models that are possible to compute with today's supercomputers excellently reproduce the total energy of a random alloy, there are limitations to the model. Using a perfectly random alloy is in it self an approximation since real alloys tend to have some degree of clustering or ordering at short-range scales. Nevertheless, the random alloy is a suitable unbiased starting point when approaching unknown alloys.

One of the limitation with the SQS method is calculating tensorial properties such as elastic constants. A recent study by Tasnádi et al. [46] pointed out a problem with obtaining tensor properties from SQS models and proposed a solution to the problem. The problem with the SQS approach is that it is not designed to preserve the point group symmetry of an alloy, and thus the tensorial properties. This has not been taken into account when generating the SQS structures used in Paper I. Therefore, the results can differ somewhat from experimental values because of the choice of SQS. However, the same SQS structure was used when comparing the elastic constants of the different alloys, therefore, any difference caused by the SQS will be present for all, and trends should not be strongly influenced.

Another limitation with the SQS method is related to the surface diffusion. The problem can be illustrated with Fig. 2.1 (b), by considering an alternative representation. In this case, the circles represent binding sites on a surface, where a fictive adatom is only allowed to move between white sites and not diagonally. Then it is clear that using this surface configuration with periodic boundary conditions will promote diffusion "highways", which in this case means that all diffusion

\footnotetext{
${ }^{1}$ Often, the term cluster is used instead of figure, which can cause some confusion.

${ }^{2}$ The SQS approach is not limited to random alloys, where $\xi_{f}^{(n)}=0$. It can also be extended to model clustering or ordering by specifying $\xi_{f}^{(n)}$ other than zero.
} 
is along the left and right direction while up and down is completely blocked. Although this example is exaggerated, it effectively illustrates the limitation of the SQS approach in modeling a property that is intrinsically long-ranged. This is one of the problems discussed in Paper VII, where we investigate the surface of an SQS model, and also one reason behind why it is important to consider the dilute limit of surface atom substitutions which is done in Paper VIII. 


\section{CHAPTER 3}

\section{Piezoelectric nitrides}

Building on the basic theoretical frameworks described in the previous chapter, this chapter covers methods used to calculated elastic and piezoelectric properties. In addition, it provides an overview of how the electromechanical properties of binary piezoelectric nitrides can be improved through alloying, and describes novel ordered piezoelectric nitrides.

\subsection{Elastic properties}

The elastic properties of a material are described by the relation between stress $\sigma_{\alpha \beta}$ and strain $\epsilon_{\gamma \delta}$. The relation between the two, and a method to calculate it using theoretical modeling, is found following Finnis [47], starting with the matrix representation of homogeneous strain

$$
\epsilon=\left(\begin{array}{lll}
\epsilon_{11} & \epsilon_{12} & \epsilon_{13} \\
\epsilon_{21} & \epsilon_{22} & \epsilon_{23} \\
\epsilon_{31} & \epsilon_{32} & \epsilon_{33}
\end{array}\right) .
$$

Assuming that the strain moves a point from $\mathbf{r}=\left(x_{1}, x_{2}, x_{3}\right)$ to the point $\mathbf{r}+\mathbf{u}$, then the elements of the matrix are defined as

$$
\epsilon_{\alpha \beta}=\frac{1}{2}\left(\frac{\partial u_{\beta}}{\partial x_{\alpha}}+\frac{\partial u_{\alpha}}{\partial x_{\beta}}\right) .
$$

The stress $\sigma_{\alpha \beta}$ is then connected via the elastic constants $C_{\alpha \beta \gamma \delta}$ as

$$
\sigma_{\alpha \beta}=\sum_{\gamma \delta} C_{\alpha \beta \gamma \delta} \epsilon_{\gamma \delta}
$$


The symmetry allows the matrices to be simplified using Voigt notation as

$$
\left(\begin{array}{l}
\epsilon_{1} \\
\epsilon_{2} \\
\epsilon_{3} \\
\epsilon_{4} \\
\epsilon_{5} \\
\epsilon_{6}
\end{array}\right)=\left(\begin{array}{c}
\epsilon_{11} \\
\epsilon_{22} \\
\epsilon_{33} \\
2 \epsilon_{23} \\
2 \epsilon_{13} \\
2 \epsilon_{12}
\end{array}\right)
$$

and

$$
\left(\begin{array}{l}
\sigma_{1} \\
\sigma_{2} \\
\sigma_{3} \\
\sigma_{4} \\
\sigma_{5} \\
\sigma_{6}
\end{array}\right)=\left(\begin{array}{l}
\sigma_{11} \\
\sigma_{22} \\
\sigma_{33} \\
\sigma_{23} \\
\sigma_{13} \\
\sigma_{12}
\end{array}\right)
$$

This makes it possible to also reduce the elastic constant matrix to a $6 \times 6$ matrix $C_{i j}$ and simplify Eq.(3.3) to

$$
\sigma_{i}=\sum_{j} C_{i j} \epsilon_{j}
$$

$C_{i j}$ can be more or less simple, because many of the components are zero due to point symmetry in the structure. For cubic crystals there are only 3 independent elastic constants $\left(C_{11}, C_{12}\right.$, and $\left.C_{44}\right)$, and for hexagonal crystals there are $5\left(C_{11}\right.$, $C_{12}, C_{13}, C_{33}$, and $C_{44}$ ). The elastic constant matrix for hexagonal crystals in full is [48]

$$
C_{i j}=\left(\begin{array}{cccccc}
C_{11} & C_{12} & C_{13} & 0 & 0 & 0 \\
C_{12} & C_{11} & C_{13} & 0 & 0 & 0 \\
C_{13} & C_{13} & C_{33} & 0 & 0 & 0 \\
0 & 0 & 0 & C_{44} & 0 & 0 \\
0 & 0 & 0 & 0 & C_{44} & 0 \\
0 & 0 & 0 & 0 & 0 & \frac{1}{2}\left(C_{11}-C_{12}\right)
\end{array}\right)
$$

The five independent matrix elements for hexagonal crystals are calculated from of the elastic energy per unit volume $U$ which is defined as [47]

$$
U=\frac{1}{2} \sum_{i j} C_{i j} \epsilon_{i} \epsilon_{j}
$$

The strain configurations $\epsilon=\left(\epsilon_{1}, \epsilon_{2}, \epsilon_{3}, \epsilon_{4}, \epsilon_{5}, \epsilon_{6}\right)$ and their corresponding elastic 
energy function, that can be used to determine the five elastic constants, are

$$
\begin{aligned}
\epsilon^{1}=(\delta, \delta, 0,0,0,0) \rightarrow U^{1} & =\left(C_{11}-C_{12}\right) \delta^{2}, \\
\epsilon^{2}=(\delta, \delta,-2 \delta, 0,0,0) \rightarrow U^{2} & =\left(C_{11}+C_{12}-4 C_{13}+2 C_{33}\right) \delta^{2}, \\
\epsilon^{3}=(0,0, \delta, 0,0,0) \rightarrow U^{3} & =\frac{1}{2} C_{33} \delta^{2}, \\
\epsilon^{4}=(0,0,0,0,0, \delta) \rightarrow U^{4} & =\frac{1}{4}\left(C_{11}-C_{12}\right) \delta^{2}, \\
\epsilon^{5}=(0,0,0, \delta, \delta, 0) \rightarrow U^{5} & =C_{44} \delta^{2},
\end{aligned}
$$

where $\delta$ is a set of small distortions of $\pm 2 \%$. The elastic constants can then be obtained by fitting a second order polynomial to the data set. Note that only $C_{33}$ and $C_{44}$ can be directly obtained from a single set of distortions, the others can only be found in combinations with other distortion sets. Of these two, $C_{33}$ is the most important in this work, since this is the elastic constant for the $c$-direction of the hexagonal crystal, which is the first part of determining the response of a piezoelectric wurtzite material.

\subsubsection{Elastic constants in disordered structures}

The SQS approach, discussed in Sec. 2.3.2, provides an excellent scheme to model the thermodynamic properties of an alloy, e.g., total energies. A good convergence of the total energy does, however, not necessarily mean a good convergence of tensorial properties such as elastic and piezoelectric constants.

Tasnádi et al. [46] show that, in case of hexagonal symmetry ${ }^{1}$, the five independent elastic coefficients are slowly converging with respect to SQS size and propose a symmetry-base projection technique to fully utilize the SQS structure. Instead they suggest the use of the projected elastic constants $\bar{C}_{11}, \bar{C}_{12}, \bar{C}_{13}, \bar{C}_{33}$, and $\bar{C}_{44}$. These require only four additional tensor coefficients, $C_{22}, C_{23}, C_{44}$, and $C_{66}$ to be calculated. The projected elastic constants can then be calculated with

$$
\begin{aligned}
& \bar{C}_{11}=3\left(C_{11}+C_{22}\right) / 8+C_{12} / 4+C_{66} / 2, \\
& \bar{C}_{12}=\left(C_{11}+C_{22}\right) / 8+3 C_{12} / 4-C_{66} / 2, \\
& \bar{C}_{13}=\left(C_{13}+C_{23}\right) / 2, \\
& \bar{C}_{33}=C_{33},
\end{aligned}
$$

and

$$
\bar{C}_{44}=\left(C_{44}+C_{55}\right) / 2 .
$$

The piezoelectric calculations reported in the papers included in this thesis (with the exception of Paper VI) have been focused on the tensorial properties along the $c$-axis, specifically the $C_{33}$ constant. This constant is not changed by the projection technique, see Eq. (3.17). Thus, no additional tensor components need to be calculated in this particular case.

\footnotetext{
${ }^{1}$ Although the cubic symmetry is the main symmetry discussed in the article, the hexagonal case is also derived in its supplementary materials.
} 


\subsection{Berry-phase theory of polarization}

The difficulty calculating polarization comes from that it is not a bulk property, which means it is dependent on the shape and truncation of the sample [49]. A more complex approach is therefore needed.

The Berry-phase approach, as stated by Vanderbilt [50], calculates the polarization in a system using geometric quantum phases known as Berry phases. The total polarization is [50]

$$
\mathbf{P}=\frac{e}{\Omega} \sum_{\tau} Z_{\tau} \mathbf{r}_{\tau}+\sum_{n \text { occ }} \mathbf{P}_{n}
$$

where $\Omega$ is the unit cell volume, $e$ is the elementary charge, $Z_{\tau}$ is the atomic number of the $\tau$-th nucleus, $\mathbf{r}_{\tau}$ its position. The first part is the contribution from the nuclei and the second part comes from the spontaneous electronic polarization of the occupied valence bands.

With Berry phases $\phi, \mathbf{P}_{n}$ can be written as

$$
\mathbf{P}_{n}=-\frac{1}{2 \pi} \frac{e}{\Omega} \sum_{\alpha} \phi_{n, \alpha} \mathbf{R}_{\alpha}
$$

where $\mathbf{R}_{\alpha}$ is a real-space primitive lattice vector corresponding to the reciprocalspace primitive lattice vector $\mathbf{G}_{\alpha}$.

$$
\phi_{n, \alpha}=\Omega_{\mathrm{BZ}}^{-1} \int_{\mathrm{BZ}} \mathrm{d}^{3} k\left\langle u_{n \mathbf{k}}\left|-i \mathbf{G}_{\alpha} \cdot \nabla_{\mathbf{k}}\right| u_{n \mathbf{k}}\right\rangle,
$$

where $\Omega_{B Z}$ is the volume of the Brillouin zone (BZ), $u_{n \mathbf{k}}=e^{i \mathbf{k} \cdot \mathbf{r}} \psi_{n \mathbf{k}}(\mathbf{r})$ is the cell-periodic Bloch functions, and $\nabla_{\mathbf{k}}$ is the k-space gradient.

For a full derivation of this approach see, e.g., Refs. [49-52].

\subsection{Piezoelectric properties}

There are four material coefficients that describe the piezoelectric properties of a material, $d_{i j}, e_{i j}, g_{i j}$, and $h_{i j}$. These connect stress $\sigma$ and strain $\epsilon$ to changes in the electrical and dielectric fields $E$ and $D$ and vice versa via the relations

$$
\begin{gathered}
d_{i j}=\left(\frac{\partial D_{i}}{\partial \sigma_{j}}\right)_{E}=\left(\frac{\partial \epsilon_{j}}{\partial E_{i}}\right)_{\sigma}, \\
e_{i j}=\left(\frac{\partial D_{i}}{\partial \epsilon_{j}}\right)_{E}=-\left(\frac{\partial \sigma_{j}}{\partial E_{i}}\right)_{\epsilon}, \\
g_{i j}=-\left(\frac{\partial E_{i}}{\partial \sigma_{j}}\right)_{D}=\left(\frac{\partial \epsilon_{j}}{\partial D_{i}}\right)_{\sigma}, \\
h_{i j}=-\left(\frac{\partial E_{i}}{\partial \epsilon_{j}}\right)_{D}=-\left(\frac{\partial \sigma_{j}}{\partial D_{i}}\right)_{\epsilon},
\end{gathered}
$$




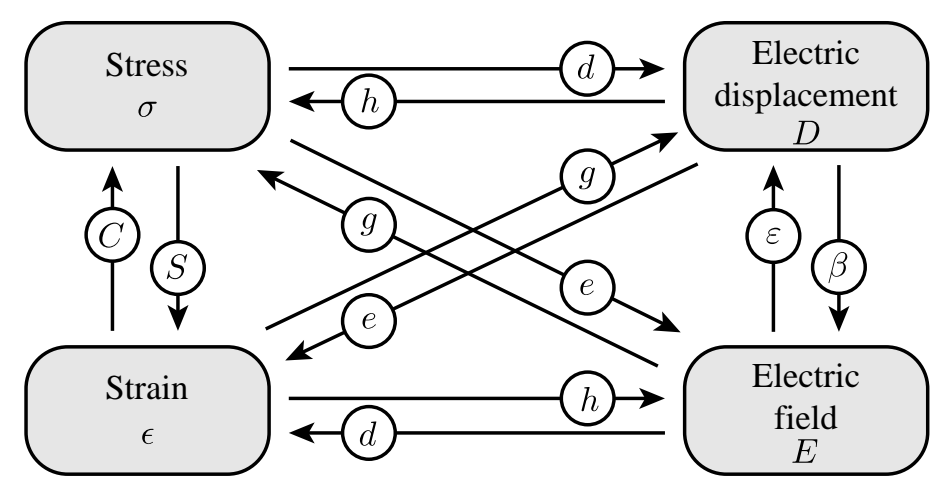

Figure 3.1. Schematic overview of the relations between piezoelectric coefficients, elastic constants, electric permittivity, stress, strain, electric displacement and electric field. Adapted from Ref.[53].

where the first part of the equations correspond to the direct piezoelectric effect, and the second part correspond to the converse piezoelectric effect [53]. A helpful schematic overview of the relations between physical properties and the piezoelectric coefficients is presented in Fig. 3.1.

The piezoelectric coefficient $e$ can be calculated within density functional theory using the Berry-phase approach suggested by Bernardini et al. [51]. This approach focuses on investigating how the polarization in the material changes when strain is applied to it.

The total polarization $\mathbf{P}$ is given by

$$
\mathbf{P}=\mathbf{P}^{e q}+\delta \mathbf{P}
$$

where $\mathbf{P}^{e q}$ is the polarization of the equilibrium structure and $\delta \mathbf{P}$ is the piezoelectric polarization. The piezoelectric part is, in the linear regime, given by

$$
\delta P_{i}=\sum_{j} e_{i j} \epsilon_{j}
$$

where $\epsilon_{j}$ is the strain component.

For wurtzite thin films, the most commonly observed growth direction is the $c$-direction. The main focus within this thesis has, therefore, been on $\delta P_{3}$, which is defined as

$$
\delta P_{3}=e_{33} \epsilon_{3}+e_{31}\left(\epsilon_{1}+\epsilon_{2}\right),
$$

where $\epsilon_{3}$ is the strain along the $c$-axis, and $\epsilon_{1}$ and $\epsilon_{2}$ are the in-plane strain.

The wurtzite structure has three independent components in the piezoelectric tensor, $e_{33}, e_{31}$, and $e_{15}$. The third component $e_{15}$ is related to shear strain. Because thin films are so flat, shear effects are much less important than deformation in the $c$-direction. 
With $c_{0}$ and $a_{0}$ as the equilibrium lattice constants, the strain components in Eq.(3.28) can be replaced with

$$
\epsilon_{3}=\frac{c-c_{0}}{c_{0}}
$$

and, assuming that $\epsilon_{1}$ and $\epsilon_{2}$ are isotropic,

$$
\epsilon_{1}=\epsilon_{2}=\frac{a-a_{0}}{a_{0}},
$$

so that the change in polarization can be be expressed as

$$
\delta P_{3}=\frac{\partial P_{3}}{\partial a}\left(a-a_{0}\right)+\frac{\partial P_{3}}{\partial c}\left(c-c_{0}\right)+\frac{\partial P_{3}}{\partial u}\left(u-u_{0}\right),
$$

where the third term takes into account changes in polarization due to internal relaxation changing the internal equilibrium parameter $u_{0}$.

When these derivatives are known, the piezoelectric coefficients can be calculated using [51]

$$
e_{33}=c_{0} \frac{\partial P_{3}}{\partial c}+\frac{4 e c_{0}}{\sqrt{3} a_{0}^{2}} Z^{*} \frac{d u}{d c}
$$

and

$$
e_{31}=\frac{a_{0}}{2} \frac{\partial P_{3}}{\partial a}+\frac{2 e}{\sqrt{3} a_{0}} Z^{*} \frac{d u}{d a},
$$

where the dynamical Born effective charge $Z^{*}$ is

$$
Z^{*}=\frac{\sqrt{3} a_{0}^{2}}{4 e} \frac{\partial P_{3}}{\partial u}
$$

The first term in Eq. (3.32) and (3.33) corresponds to the clamped-ion term, which represent the effect of the strain on the electronic structure. ${ }^{2}$ The second term corresponds to the change in polarisation with respect to changes in the internal parameter $u$.

The piezoelectric coefficient $e_{i j}$ cannot be directly obtained experimentally. Therefore, it is useful to convert $e_{i j}$ to the corresponding $d_{i j}$. The relation between the relevant components are given by [54]

$$
e_{31}=d_{31}\left(C_{11}+C_{12}\right)+d_{33} C_{13}
$$

and

$$
e_{33}=2 d_{31} C_{13}+d_{33} C_{33} .
$$

Using these relations, $d_{33}$ can be written as

$$
d_{33}=\frac{e_{33}-\frac{2 C_{13}}{C_{11}+C_{12}} e_{31}}{C_{33}-\frac{2 C_{13}^{2}}{C_{11}+C_{12}}} .
$$

\footnotetext{
${ }^{2}$ Note that $e$ without indices is the elementary charge and should not be mistaken for the piezoelectric tensor.
} 
The alloys investigated within the scope of this thesis are thin films. For these, it is likely that the in-plane strain is somewhat clamped by the substrate, so that $C_{11}, C_{12} \rightarrow \infty$. The effect of this clamping due to a rigid substrate has also been investigated experimentally by Dubois \& Muralt [55]. We, therefore, define the clamped piezoelectric response $d_{33, f}$ as

$$
d_{33, f}=\frac{e_{33}}{C_{33}},
$$

Moreover, for $\mathrm{Sc}_{x} \mathrm{Al}_{1-x} \mathrm{~N}, e_{31}$ is strictly negative in the relevant composition range and the elastic constants are all positive [56]. This is likely the same for the other piezoelectric wurtzite nitrides investigated in Papers I, II, III, and V. Thus, it is evident that

$$
d_{33} \geq d_{33, f}
$$

which means that $d_{33, f}$ is a lower bound for $d_{33}$, and of high relevance for $c$ oriented thin films. Furthermore, $d_{33, f}$ is much less computationally demanding which is useful when comparing the effects of chemistry, composition, etc., in many different alloys. Thus, primarily studying the three coefficients $C_{33}, e_{33}$, and $d_{33, f}$ is an effective starting point to find new piezoelectric alloys with similar or greater increases than previously known cases at lower computational cost than calculating all of the tensor coefficients of the three properties.

In modern ab initio codes, e.g. the Vienna ab-initio simulation package (VASP), there are automatic methods implemented that can be used to calculate the full piezoelectric response. These methods are usually based on density functional perturbation theory [57] or finite difference techniques. Calculating the full piezoelectric tensor for systems with more than 100 atoms, however, requires a large amount of computational resources. Therefore, the accuracy of the calculations needs to be sacrificed in order to obtain results. When investigating trends in multiple alloys this is currently too computationally demanding. However, after finding a material which fulfills a specific criteria, the full piezoelectric tensor can be calculated to provide the complete picture.

\subsection{Increasing the piezoelectric response of AlN}

AlN is a useful piezoelectric material by itself. However, by alloying AlN with ScN, Akiyama et al. (2009) found that the piezoelectric response of AlN could be increased by $\sim 400 \%$ at a $43 \% \mathrm{ScN}$ concentration [11]. The microscopic origin of this effect was later studied by Tasnádi et al. [58], who found that the increase in the piezoelectric response of the $\mathrm{Sc}_{1-x} \mathrm{Al}_{x} \mathrm{~N}$ alloy is caused by the similarity between the parent wurtzite phase of AlN and the layered hexagonal phase of $\mathrm{ScN}$, two very similar phases (see Fig. 3.2). The only difference between the two structures is that in the layered hexagonal phase the Sc and $\mathrm{N}$ ions are in the same $c$-plane, $u=0.5$, which means that the in-plane distances are larger and the layers are more closely packed, i.e., a lower $c / a$-ratio. The similarity of the structures leads to a phase competition caused by a morphotropic phase boundary [59] that separate the polar wurtzite and non-polar layered-hexagonal phases of 
(a) Wurtzite AIN

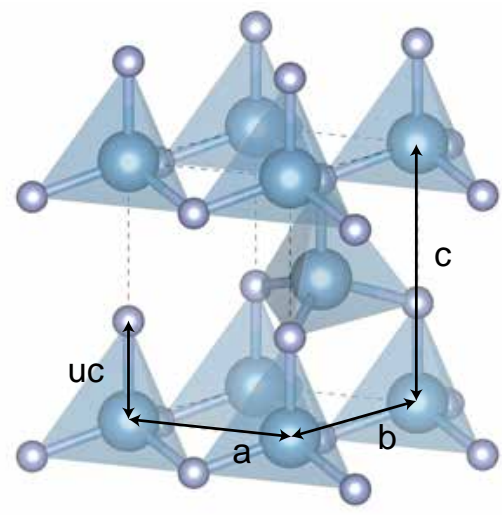

(b) Layered hexagonal ScN

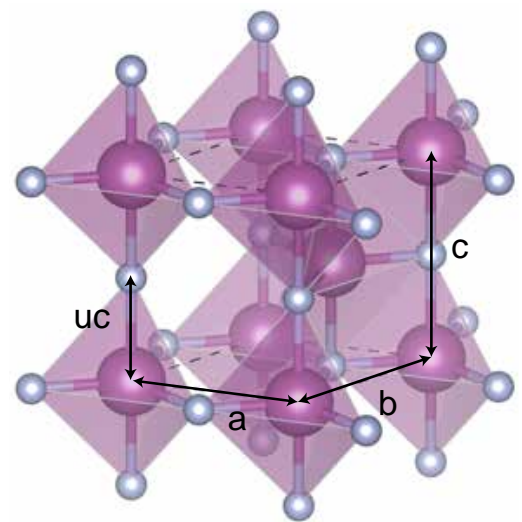

Figure 3.2. (a) The AlN wurtzite crystal structure, with the in plane lattice parameters $a$ and $b$, out of plane parameter $c$, and internal parameter $u$. (b) The ScN layered hexagonal crystal structure. $\mathrm{N}$ atoms from neighboring unit cells are added to visualize the local tetrahedral and octahedral environments.

the material. The resulting instability leads to the increase of $e_{33}$ and decrease of $C_{33}$, simultaneously.

Tasnádi et al. [58] also show that the phase competition in $\mathrm{Sc}_{x} \mathrm{Al}_{1-x} \mathrm{~N}$ is characterized by a flattening of the energy surface compared to pure AlN. The flat energy surface has a low second derivative with respect to distortions in the $c$ direction, which is directly proportional to the low elastic constant. Moreover, the $c / a$ of the wurtzite minimum is shifted some toward lower $c / a$ and the layered hexagonal structure.

Furthermore, Tasnádi et al. [58] point out that the phase competition they studied in $\mathrm{Sc}_{x} \mathrm{Al}_{1-x} \mathrm{~N}$ can be used as a criteria to search for in order to discover new material systems with substantially increased piezoelectric response.

\subsection{Expanding the search for piezoelectric ternary nitrides}

The origin of the increased piezoelectric response in $\mathrm{Sc}_{1-x} \mathrm{Al}_{x} \mathrm{~N}$, inspired the work in Paper I. There, we investigate if the Sc effect is transferable to similar wurtzite nitrites, i.e., GaN and $\mathrm{InN}$, and if it is possible to use an alternative group 3 alloying component, $\mathrm{Y}$, to produce similar results. In order to minimize the computational effort, the calculations were limited to the $x=0.5$, where the highest piezoelectric response is anticipated. 


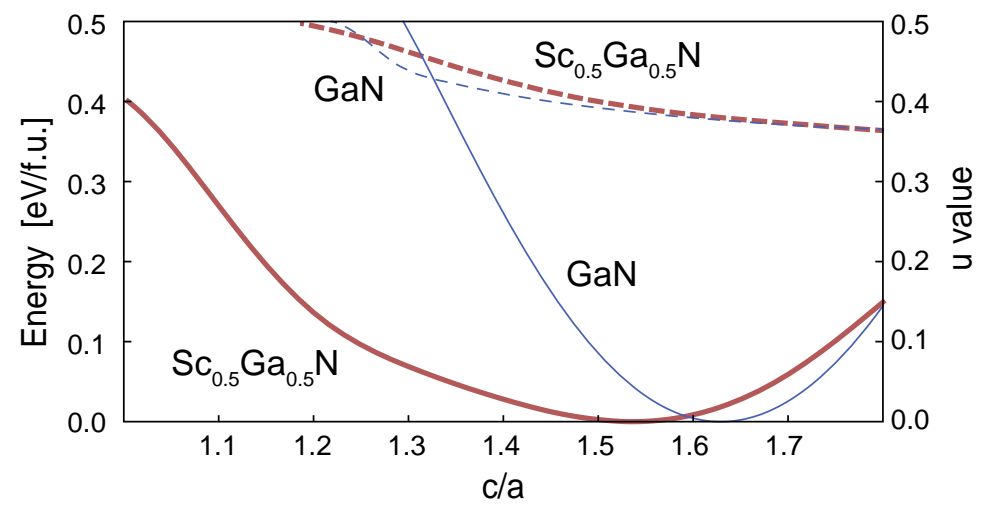

Figure 3.3. $\mathrm{Sc}_{0.5} \mathrm{Ga}_{0.5} \mathrm{~N}$ and $\mathrm{GaN}$ minimum energy path along $c / a$. Remodeled from Paper I.

\subsubsection{Improvement of the piezoelectric response}

The similarities to $\mathrm{Sc}_{x} \mathrm{Al}_{1-x} \mathrm{~N}$ are apparent when looking at the energy surfaces of the similar alloys. Fig. 3.3 shows the cross-section of ScGaN and GaN energy landscapes along the minimum energy path in the $c / a$ direction. Here, we can see the same effects as in $\mathrm{Sc}_{x} \mathrm{Al}_{1-x} \mathrm{~N}$, that the wurtzite minimum is flattened and shifted toward lower $c / a$ ratio. The degree of flattening and the shift in $c / a$ differs between the alloys together with their piezoelectric and elastic properties.

The calculations in Paper I show that all $\mathrm{A}_{0.5} \mathrm{~B}_{0.5} \mathrm{~N}(\mathrm{~A}=\mathrm{Sc}, \mathrm{Y} ; \mathrm{B}=\mathrm{Al}, \mathrm{Ga}$, In) alloys have improved piezoelectric response compared to their parent wurtzite compound, see Tables 3.1, 3.2, and 3.3. Although all alloys have noteworthy increases the range of the improvement is large, from $86 \%$ increase for $\mathrm{Sc}_{0.5} \operatorname{In}_{0.5} \mathrm{~N}$ up to $811 \%$ for $\mathrm{Sc}_{0.5} \mathrm{Ga}_{0.5} \mathrm{~N}$. The highest piezoelectric response is, however, still in $\mathrm{Sc}_{0.5} \mathrm{Al}_{0.5} \mathrm{~N}$ due to the low piezoelectric response in pure GaN.

The alloys all exhibit the same characteristics behind the increase of the piezoelectric response, an increased $e_{33}$ and a decreased $C_{33}$, regardless of the alloying component. It is also clear from the data in Tables 3.1, 3.2, and 3.3 that $\mathrm{Sc}$ is not always the best alloying component. Of the $\mathrm{InN}$ alloys, $\mathrm{Y}_{0.5} \operatorname{In}_{0.5} \mathrm{~N}$, has the highest piezoelectric response.

The results in Table 3.1 also show that the piezoelectric response is very sensitive to the approach. The difference in the properties between QuantumESPRESSO (QE) and VASP calculations using the same SQS is less than $15 \%$ for $e_{33}$ and $C_{33}$, while the difference in $d_{33}$ can be significantly larger since it is a combination of the other two. Many things differ between these results, the program itself and the pseudopotential approach. The QE calculations were performed using ultrasoft pseudopotentials and the VASP calculations used PAW pseudopotentials. QE has since the calculations were performed gone through many code updates and the pseudopotentials have been improved, thus it would be interesting to compare the approaches to each other with the modern codes and potentials. 


\begin{tabular}{l|ccc}
\hline \hline Material & $e_{33}\left(\mathrm{C} / \mathrm{m}^{2}\right)$ & $C_{33}(\mathrm{GPa})$ & $d_{33, f}(\mathrm{pC} / \mathrm{N})$ \\
\hline AlN (exp.) & $1.5[60]$ & $375^{a}$ & $4.0[60]$ \\
AlN (QE) & 1.38 & 342.0 & 4.03 \\
AlN (VASP2) & 1.46 & 345.1 & 4.23 \\
$\mathrm{Sc}_{0.5} \mathrm{Al}_{0.5} \mathrm{~N}(\mathrm{QE})$ & 3.05 & 130.0 & 23.46 \\
$\mathrm{Sc}_{0.5} \mathrm{Al}_{0.5} \mathrm{~N}$ & 3.16 & 120.7 & 26.20 \\
$(\mathrm{VASP} 1)$ & & & \\
$\mathrm{Sc}_{0.5} \mathrm{Al}{ }_{0.5} \mathrm{~N}$ & 3.33 & 114.8 & 29.04 \\
$(\mathrm{VASP} 2)$ & 2.15 & 171.4 & 12.54 \\
$\mathrm{Y}_{0.5} \mathrm{Al}_{0.5} \mathrm{~N}(\mathrm{QE})$ & 2.46 & 145.9 & 16.87 \\
$\mathrm{Y}_{0.5} \mathrm{Al}_{0.5} \mathrm{~N}$ & & & \\
$(\mathrm{VASP} 2)$ &
\end{tabular}

Table 3.1. Piezoelectric and elastic coefficients of AlN together with the piezoelectric response when alloyed with $50 \% \mathrm{Sc}$ or Y. QE results were calculated with QuantumESPRESSO and the SQS from Paper I. VASP1 results were obtained using VASP and the SQS from Paper I. VASP2 results were calculated using VASP and the SQS from Paper V. ${ }^{a}$ Calculated from the experimental $d_{33}$ and $e_{33}$ values.

\begin{tabular}{l|ccc}
\hline \hline Material & $e_{33}\left(\mathrm{C} / \mathrm{m}^{2}\right)$ & $C_{33}(\mathrm{GPa})$ & $d_{33, f}(\mathrm{pC} / \mathrm{N})$ \\
\hline GaN (exp.) & $0.85[60]$ & $303^{a}$ & $2.8[60]$ \\
GaN (QE) & 0.66 & 357.7 & 1.86 \\
$\mathrm{Sc}_{0.5} \mathrm{Ga}_{0.5} \mathrm{~N}(\mathrm{QE})$ & 2.30 & 135.9 & 16.90 \\
$\mathrm{Y}_{0.5} \mathrm{Ga}_{0.5} \mathrm{~N}(\mathrm{QE})$ & 1.85 & 191.0 & 9.67 \\
\hline \hline
\end{tabular}

Table 3.2. Piezoelectric and elastic coefficients of GaN together with the piezoelectric response when alloyed with $50 \% \mathrm{Sc}$ or Y. QE results were calculated with QuantumESPRESSO and the SQS from Paper I. VASP1 results were obtained using VASP and the SQS from Paper I. ${ }^{a}$ Calculated from experimental $d_{33, f}$ and $e_{33}$ values.

An other key aspect that influences the piezoelectric response is the SQS used to model the alloy, see for example the $\mathrm{Sc}_{0.5} \mathrm{Al}_{0.5} \mathrm{~N}$ results for VASP1 and VASP2 in Table 3.1. These two results differ by $\sim 11 \%$. In a resent study by Caro et al. [56] they show that there is a large difference is the piezoelectric response depending on the structure, where $e_{33}$ and $C_{33}$ can differ by as much as $\sim 25 \%$ for random alloys with 128 atoms. While the error from the SQS approach is likely smaller, it is important to take this into account. Thus, for comparisons one should use the same supercell inorder to reduce the influence the of the SQS.

Caro et al. [56] also calculate the full $d_{33}$ for $\mathrm{Sc}_{x} \mathrm{Al}_{1-x} \mathrm{~N}$ without any clamping. They find that $d_{33}$ is almost $2 \times d_{33, f}$ at $x=0.5$. At lower $x$ the difference is much smaller, e.g., at $x=0.25$ the difference is only $40 \%$. Compared to the experimental data they present, however, $d_{33, f}$ is the closest fit to the experimental data. Thus, indicating that the thin films are indeed severely clamped. 


\begin{tabular}{l|ccc}
\hline \hline Material & $e_{33}\left(\mathrm{C} / \mathrm{m}^{2}\right)$ & $C_{33}(\mathrm{GPa})$ & $d_{33, f}(\mathrm{pC} / \mathrm{N})$ \\
\hline InN (exp.) & $0.57^{a}$ & $182[61]$ & $3.12[62]$ \\
InN (QE) & 1.09 & 211.4 & 5.18 \\
InN (VASP1) & 0.94 & 204.9 & 4.60 \\
$\mathrm{Sc}_{0.5} \operatorname{In}_{0.5} \mathrm{~N}(\mathrm{QE})$ & 1.47 & 153.1 & 9.60 \\
$\mathrm{Y}_{0.5} \operatorname{In}_{0.5} \mathrm{~N}(\mathrm{QE})$ & 2.26 & 107.1 & 21.10 \\
$\mathrm{Y}_{0.5} \mathrm{In}_{0.5} \mathrm{~N}$ & 2.20 & 94.3 & 23.3 \\
$(\mathrm{VASP} 1)$ & & & \\
\hline \hline
\end{tabular}

Table 3.3. Piezoelectric and elastic coefficients of $\mathrm{InN}$ together with the piezoelectric response when alloyed with $50 \% \mathrm{Sc}$ or Y. QE results were calculated with QuantumESPRESSO and the SQS from Paper I. VASP1 results were obtained using VASP and the SQS from Paper I. ${ }^{a}$ Calculated from experimental $d_{33, f}$ and $e_{33}$ values.

\subsubsection{A volume matching condition}

It would be highly valuable to be able to predict which alloy combinations that have large increase in the piezoelectric response without calculating energy surfaces or piezoelectric coefficients. It would save a lot of computational resources by limiting time consuming detailed investigations to the most likely candidate alloys when searching for new piezoelectric materials. In Paper I, our study of AlN, GaN and InN alloyed with Sc or $\mathrm{Y}$ revealed that it is important to match the unit cell volumes of the parent binary nitrides, see Fig. 3.4. The highest increases in the piezoelectric responses, marked with thick green arrows, is found in the alloys where the unit cell volume difference is smallest.

In alloy combinations where the volume difference between the parent binary alloying components is small, the wurtzite and layered hexagonal phases are closer to each other structurally. This increases the phase competition between the wurtzite and layered hexagonal phases. The energy landscapes of these alloys are, thus, flatter.

Finding the equilibrium volume of a structure is a comparably much less demanding task than calculating the full piezoelectric tensor. Thus, this can be used as an initial criteria to focus on alloys where the unit cell difference between the alloying components is not too large.

\subsection{3 $\quad \mathrm{Y}_{1-x} \operatorname{In}_{x} \mathrm{~N}$}

In Paper I, we pointed out that $\mathrm{Y}_{1-x} \mathrm{In}_{x} \mathrm{~N}$ is an interesting alternative to $\mathrm{Sc}_{1-x} \mathrm{Al}_{x} \mathrm{~N}$ because of its high piezoelectric response. This led to the study in Paper III, where we investigated the electromechanical properties in the composition range $0 \leq x \leq 0.5$, see Fig. 3.5, and performed synthesis and characterization experiments.

Compared to $\mathrm{Sc}_{x} \mathrm{Al}_{1-x} \mathrm{~N}, e_{33}$ of $\mathrm{Y}_{x} \mathrm{In}_{1-x} \mathrm{~N}$ is about $0.5 \mathrm{C} / \mathrm{m}^{2}$ lower throughout the investigated composition range. Although this lowers the piezoelectric response, at the same time, $C_{33}$ is also much lower, which has the opposite effect. 


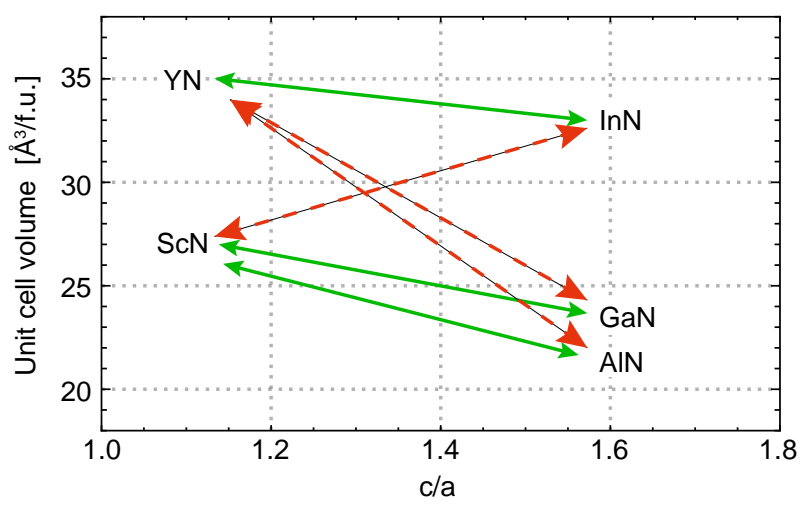

Figure 3.4. Unit cell volume and $c / a$ ratio of binary compounds used in the piezoelectric alloys investigated in Paper I. The green thick arrows mark the combinations with the highest increase for AIN, GaN, and InN. Remodeled from Paper I.
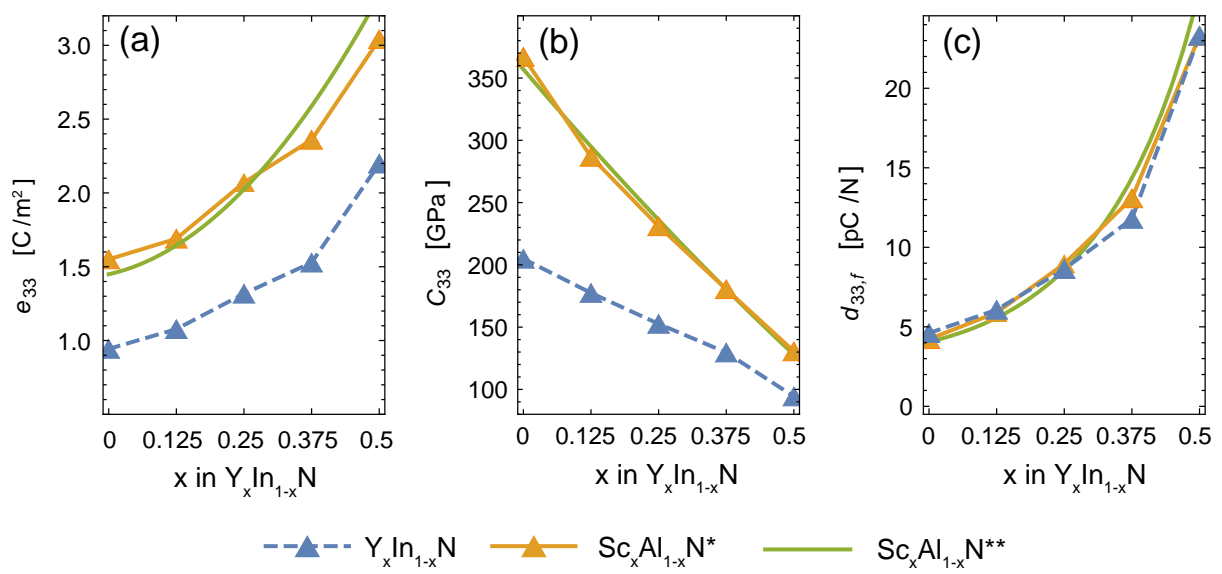

Figure 3.5. Electromechanical properties of $\mathrm{Y}_{x} \operatorname{In}_{1-x} \mathrm{~N}$, (a) $e_{33}$, (b) $C_{33}$, and (c) $d_{33, f}$, with $\mathrm{Sc}_{x} \mathrm{Al}_{1-x} \mathrm{~N}$ values from ${ }^{*}$ Ref. [58], and ${ }^{* *}$ Ref. [56]. Remodeled from Paper III. 


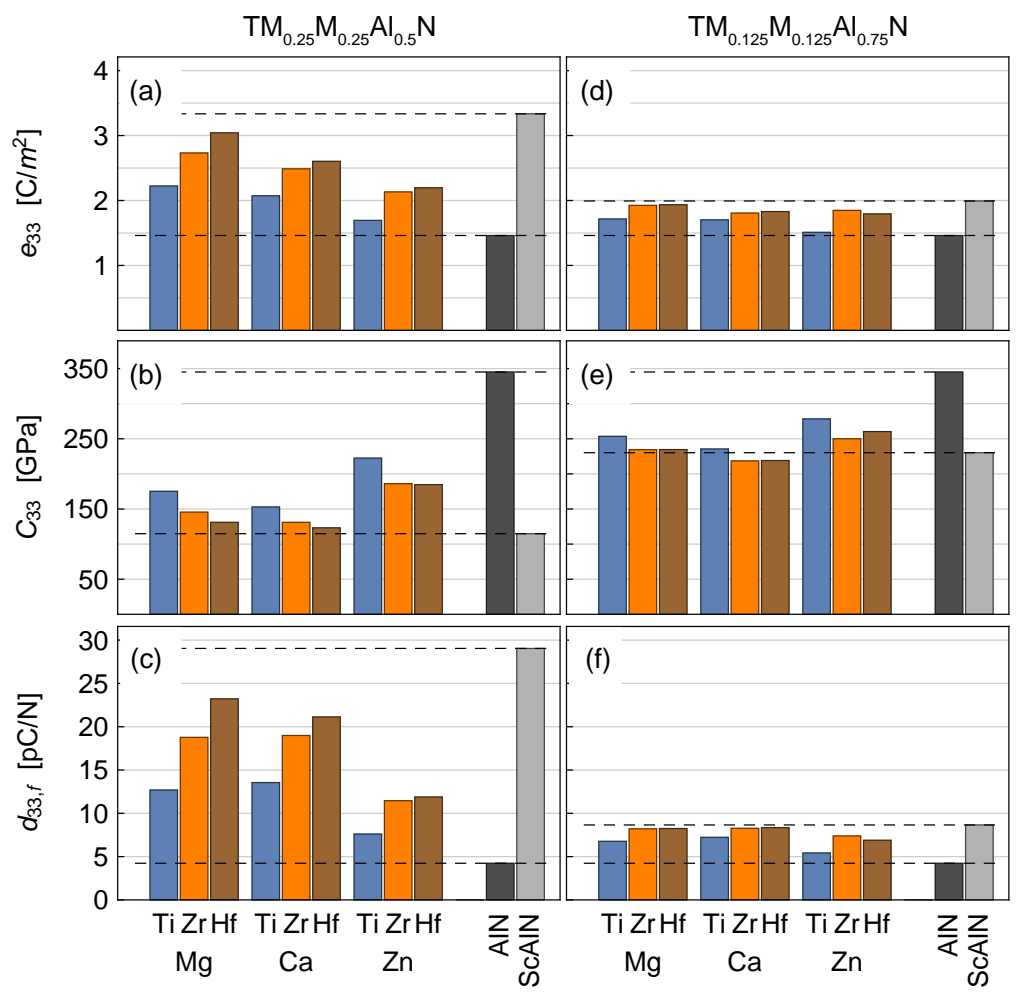

Figure 3.6. The electromechanical properties $e_{33}, C_{33}$, and $d_{33, f}$ of quarternary wurtzite $\mathrm{TM}_{x / 2} \mathrm{M}_{x / 2} \mathrm{Al}_{1-x} \mathrm{~N}$ alloys for $x=0.5$ and 0.25 . AlN and concentration specific ScAlN values are added as references. Remodeled from Paper $\mathrm{V}$.

The combination of the two effects results in a similar piezoelectric response in both $\mathrm{Sc}_{x} \mathrm{Al}_{1-x} \mathrm{~N}$ and $\mathrm{Y}_{x} \mathrm{In}_{1-x} \mathrm{~N}$. Thus, the potential of $\mathrm{Y}_{x} \mathrm{In}_{1-x} \mathrm{~N}$ for piezoelectric application, is substantial.

\subsection{Expanding the search to quarternary alloys}

In Paper $\mathrm{V}$ we widen our search for piezoelectric wurtzite crystal structure nitrides to quarternary alloys. Inspired by Alling [63] who showed that it is possible to create a thermodynamically stable semiconducting nitride alloy in disordered $\mathrm{Ti}_{0.5} \mathrm{Mg}_{0.5} \mathrm{~N}$ and ordered $\mathrm{TiMgN} \mathrm{N}_{2}$, we considered that equal amounts of $\mathrm{Ti}^{4+}$ with $\mathrm{Mg}^{2+}$ could exhibit similar properties as $\mathrm{Sc}^{3+}$ and $\mathrm{Y}^{3+}$ when alloying with $\mathrm{AlN}$, because of the valence matching. Thus, we investigated multiple group 2 and 4 elements, namely $\mathrm{TM}_{x / 2} \mathrm{M}_{x / 2} \mathrm{Al}_{1-x} \mathrm{~N}(\mathrm{TM}=\mathrm{Ti}, \mathrm{Zr}, \mathrm{Hf} ; \mathrm{M}=\mathrm{Mg}, \mathrm{Ca})$, and their piezoelectric properties. In addition to these alloys we also investigated combinations with $\mathrm{Zn}$ which has the same number of valence electrons as the group 2 elements. 


\subsubsection{Improvement of the piezoelectric response}

All $\mathrm{TM}_{x / 2} \mathrm{M}_{x / 2} \mathrm{Al}_{1-x} \mathrm{~N}$ ( TM = Ti, Zr, Hf; $\mathrm{M}=\mathrm{Mg}$, Ca, Zn) alloys have improved piezoelectric and elastic properties compared to pure AlN in both considered concentrations, $x=0.5$ and $x=0.25$, see Fig. 3.6 (a) - (f). Moreover, the increases arise from an increased $e_{33}$ and decreased $C_{33}$, as in the ternary alloys.

The trend of the increase is exponential with composition, like in the ternary alloys, with the highest increase at $x=0.5$. The magnitude of the maximum piezoelectric responses of the quarternary alloys at $x=0.5$ are not as high as in $\mathrm{Sc}_{x} \mathrm{Al}_{1-x} \mathrm{~N}$, e.g., $d_{33}$ of $\mathrm{Hf}_{0.25} \mathrm{Mg}_{0.25} \mathrm{Al}_{0.5} \mathrm{~N}$ is $80 \%$ that of $\mathrm{Sc}_{0.5} \mathrm{Al}_{0.5} \mathrm{~N}$. Moreover, at $x=0.25$ all but the $\mathrm{Zn}$ and $\mathrm{Ti}$ alloys are comparable to $\mathrm{Sc}_{0.25} \mathrm{Al}_{0.75} \mathrm{~N}$. At this concentration the piezoelectric response is approximately two times that of pure AlN. Thus, there is great potential for future devices based on quarternary piezoelectric nitrides, that furthermore has the advantage to be based solely on earth-abundant materials, e.g., $\mathrm{Ti}, \mathrm{Mg}, \mathrm{Al}$, and $\mathrm{N}$.

\subsubsection{Descriptors for good alloying components for AlN}

In an attempt to identify what characterizes the best $\mathrm{TM}_{0.5} \mathrm{M}_{0.5} \mathrm{~N}$ alloying component for AlN we first investigated the role of volume matching, see Fig 3.7 (a). Here, it is apparent that the volume difference previously proposed is insufficient to predict the best alloying component in this extended chemical space. The smallest volume difference does not equal the highest piezoelectric response, instead there is an optimum near the $\mathrm{ScN}$ volume. The volume difference can, however, not be too large so the volume matching condition is still useful to discard alloying components with too large volume difference. Our calculations did, however, reveal a new descriptor related to the energy difference between fourfold and fivefold structures of the pure alloying component added to AlN, see Fig. 3.7 (b).

The phase competition between the fourfold and fivefold structures of the wurtzite and layered-hexagonal phases are, as discussed previously in the chapter, the reason behind the substantial increase in the piezoelectric response. Here, our focus lies on finding the best layered-hexagonal component, it is, therefore, not a stretch to imagine that the best choice would be the one with the strongest preference for the fivefold phase. The natural choices for the fourfold and fivefold representative structures are the wurtzite and the layered-hexagonal structures. However, these structures are very similar, and the one with the highest energy becomes unstable during geometry optimization and relaxes to the other. Therefore, we use the cubic zincblende phase, which has only a different layering than wurtzite, to represent the tetrahedrally coordinated phase.

The energy difference between the zincblende and the layered-hexagonal phases in the alloying component strongly correlates with the resulting piezoelectric response of the quarternary alloy, see Fig. 3.7 (b). Therefore, we propose this energy difference as a descriptor in future high-throughput searches in an even more extended chemical space of wurtzite semiconductors. 


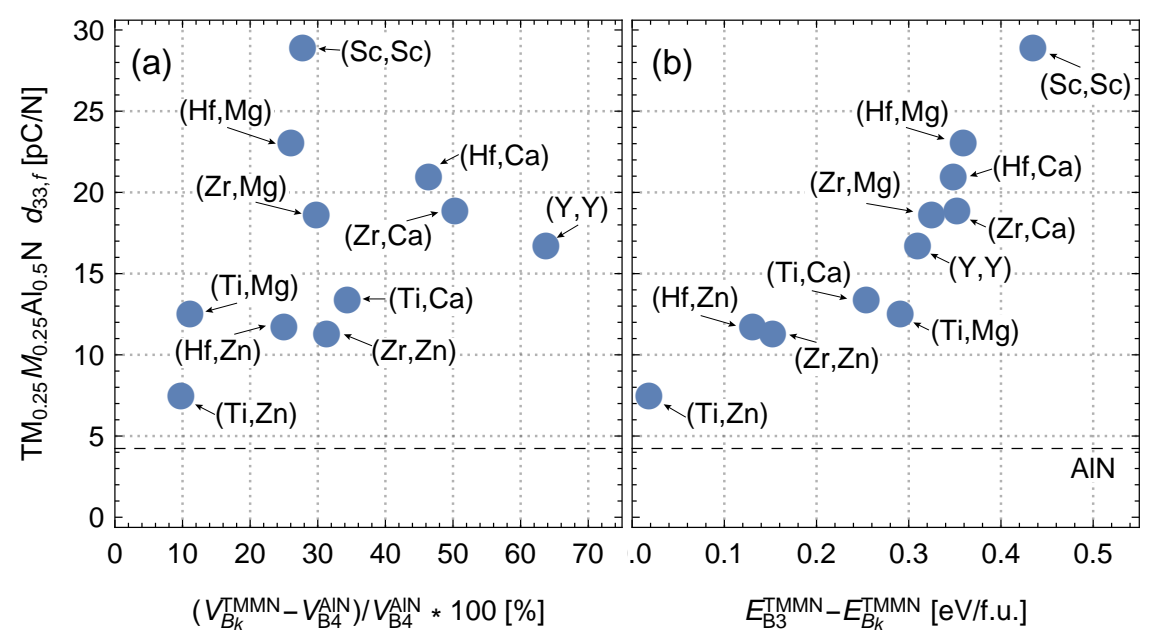

Figure 3.7. $d_{33, f}$ of different AlN alloys, in (a) with respect to the unit cell volume difference, and in (b) with respect to the layered-hexagonal and zincblende energy difference in the pure alloying component. Remodeled from the data in Paper $\mathrm{V}$ with the exception of the $(\mathrm{Y}, \mathrm{Y})$ data points. The $(\mathrm{Y}, \mathrm{Y})$ data points from literature have been replaced with new calculated values using the same method and SQS as the rest of the data points.

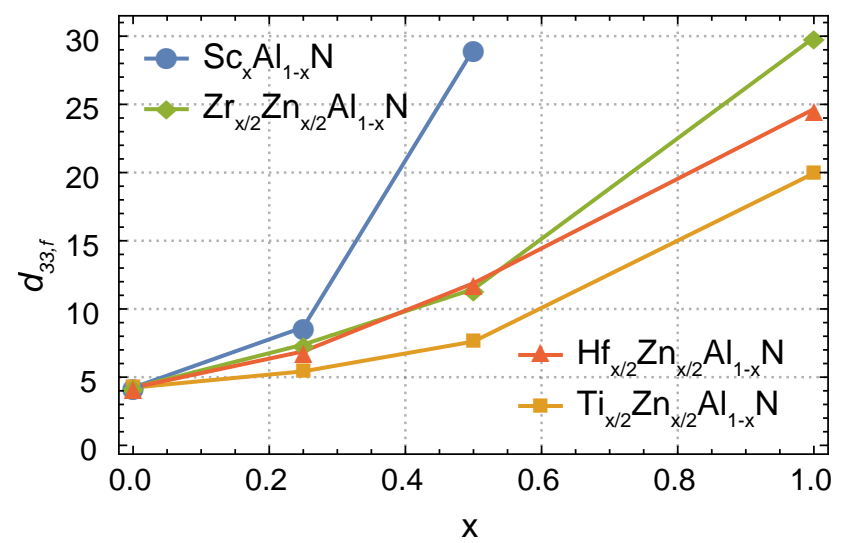

Figure 3.8. $d_{33}$ of $\mathrm{TM}_{x / 2} \mathrm{Zn}_{x / 2} \mathrm{Al}_{1-x} \mathrm{~N}(\mathrm{TM}=\mathrm{Ti}, \mathrm{Zr}, \mathrm{Hf})$ and $\mathrm{Sc}_{x} \mathrm{Al}_{1-x} \mathrm{~N}$ in the full composition range $(0 \leq x \leq 1)$. Using data presented in Paper $\mathrm{V}$. 


\section{7 $\quad \mathrm{TM}_{0.5} \mathrm{Zn}_{0.5} \mathrm{~N}$ alloys}

The $\mathrm{TM}_{0.5} \mathrm{Zn}_{0.5} \mathrm{~N}(\mathrm{TM}=\mathrm{Ti}, \mathrm{Zr}$, Hf) alloying components investigated in Paper $\mathrm{V}$ exhibit a much lower increase in the piezoelectric response compared to the other investigated components with $\mathrm{Mg}$ or Ca, see Figs. 3.6 (a) and (b). This is because the wurtzite phase is lowest in energy among the investigated disordered rocksalt, zincblende, wurtzite, and layered-hexagonal phases, for all $\mathrm{TM}_{0.5} \mathrm{Zn}_{0.5} \mathrm{~N}$. Thus, there is much weaker phase competition from the layered-hexagonal phase in the alloying component compared to the other alloys. The reason behind the increased piezoelectric response is the increasing amount of $\mathrm{TM}_{0.5} \mathrm{Zn}_{0.5} \mathrm{~N}$ which has a much better piezoelectric response than AlN.

By comparing $\mathrm{Sc}_{x} \mathrm{Al}_{1-x} \mathrm{~N}$ to the $\mathrm{TM}_{x / 2} \mathrm{Zn}_{x / 2} \mathrm{Al}_{1-x} \mathrm{~N}$ in the full composition range from 0 to 1 , see Fig. 3.8, it is evident that the piezoelectric response of the wurtzite crystal structure- $\mathrm{TM}_{0.5} \mathrm{Zn}_{0.5} \mathrm{~N}$ alloys have a comparable piezoelectric response to that of $\mathrm{Sc}_{x} \mathrm{Al}_{1-x} \mathrm{~N}$. Accordingly, it would be better to synthesize $\mathrm{TM}_{0.5} \mathrm{Zn}_{0.5} \mathrm{~N}$ alloys by themselves rather than in alloys with $\mathrm{AlN}$.

Although the piezoelectric response of these alloys are especially promising, it is not currently known if it is possible to synthesize these alloys in the wurtzite phase or at all. In order to predict if it is possible to synthesize them and in which way, we can study the thermodynamics of these alloy systems. The details of this will be covered in the next chapter. 


\section{CHAPTER 4}

\section{Thermodynamics of materials}

This chapter covers the basic definitions of thermodynamical stability and describe how theoretical tools, specifically DFT, can be used to study thermodynamical stability of materials. In addition, the phase stability of ternary ( $\mathrm{Ti}, \mathrm{Zr}, \mathrm{Hf}$ )-Zn-N is discussed.

\subsection{Gibb's free energy}

For a fixed temperature, pressure, and with a constant number of particles, a material is in thermodynamic equilibrium when it is in a global minimum in the Gibb's free energy $G$, which is defined as [64]

$$
G=H-T S \text {. }
$$

Here $T$ is the temperature, $S$ is the entropy. The enthalpy $H$ is given by

$$
H=E+P V,
$$

where $E$ is the total energy, $P$ is the pressure, and $V$ is the volume.

A common way to improve the properties of a material is to alloy it with different concentrations $x$ of another element. However, not all concentrations produce a stable alloy. It is therefore advantageous to determine the stability of a mixture by calculating the mixing free energy $\Delta G_{\text {mix }}$, according to

$$
\Delta G_{\text {mix }}(x)=G(x)-(x G(B)+(1-x) G(A)),
$$

where $G(A)$ is the free energy of element $\mathrm{A}, G(B)$ is the free energy of element B. Furthermore, $\Delta G_{\text {mix }}$ can be divided as

$$
\Delta G_{\text {mix }}=\Delta H_{\text {mix }}-T \Delta S_{\text {mix }},
$$


(a)

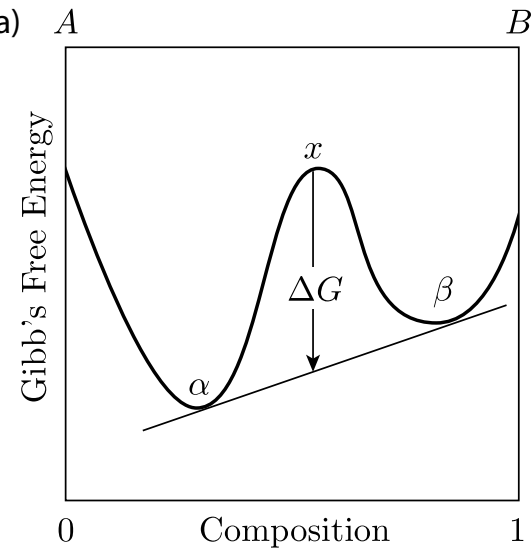

(b) $A$

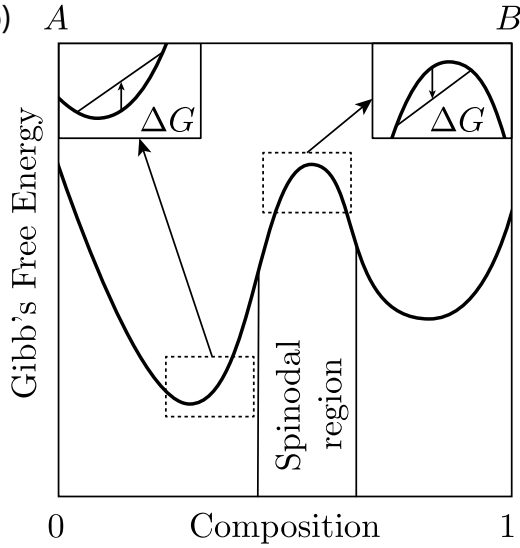

Figure 4.1. Gibb's free energy for a fictive binary alloy at a given temperature and pressure. (a) Reduction of the free energy with $\Delta G$ when the system with composition $x$ reaches equilibrium, which is a mixture of the two phases $\alpha$ and $\beta$ found by a common tangent construction. (b) Free energy differences caused by small fluctuations in the local composition.

where the contribution comes from the mixing enthalpy $\Delta H_{\text {mix }}$ and the mixing entropy $\Delta S_{\text {mix }}$.

The Gibb's free energy with respect to concentration for a fictive binary system, at some particular temperature and pressure, is presented in Fig. 4.1. In part (a) of the figure is an illustration of the ground state configuration of a mixture with the concentration $x$. From a homogeneous mixture at $x$, the material will reduce $\Delta G_{\text {mix }}$ by decomposing and forming phases with a local compositions of $\alpha$ and $\beta$.

The mechanism through which the decomposition proceed is related to the second derivative of the free energy. If $d^{2} G / d x^{2}>0$, then small fluctuations in $x$ will lead to an increase in the free energy. The decomposition is therefore driven by nucleation and growth and limited by an interface related nucleation barrier. Due to this barrier the system can be considered as metastable in the thermodynamic sense. In the opposite case when $d^{2} G / d x^{2}<0$, within the spinodal region, small fluctuations in $x$ lead to a decrease in the free energy. The mechanism behind the decomposition in this region is called spinodal decomposition, and is not required to overcome a nucleation barrier.

It is worth to point out that the fictive binary alloy in Fig. 4.1 is a simple example. In addition to the alloys in solid solution form, there can also exist relevant ordered phases stable only at very narrow composition intervals. For example, in nitrides it is very unfavorable to interchange the positions of a nitrogen atom and a metal atom. If also vacancies are unfavorable, the composition can hardly be varied at all and a so called line compound is the result. One such example is AlN. 


\subsubsection{Approximating Gibb's free energy for non-equilibrium conditions}

There are many contributing factors to Gibb's free energy, e.g., electronic, magnetic, vibrational, and configurational dependencies. Taking these into account, Gibb's free energy can be decomposed into

$$
\begin{aligned}
G=H_{0}+H^{\mathrm{el}}(T)+H^{\mathrm{mag}} & (T)+H^{\mathrm{vib}}(T)+H^{\mathrm{conf}}(T) \\
& -T\left(S^{\mathrm{el}}(T)+S^{\mathrm{mag}}(T)+S^{\mathrm{vib}}(T)+S^{\mathrm{conf}}(T)\right),
\end{aligned}
$$

where $H_{0}$ is the ground state enthalpy. Of these, the temperature dependence is the largest challenge for first-principles phase stability calculation since it requires computationally resource heavy and technically difficult methods, e.g., molecular dynamics (MD) or Monte Carlo (MC) techniques that samples temperature dependence in a population of exited states. Moreover, their contributions to the temperature dependent free energy can be large. However, when calculating formation or mixing energies, these can largely cancel out, as has been observed [65].

Furthermore, under the low temperature non-equilibrium conditions of PVD nitride thin film growth, long range diffusion is quenched and phase separation severely limited. Instead, it is more useful to study possible competing alloy phases, e.g., wurtzite or rocksalt, at each investigated composition.

The temperature dependent vibrational free energy can of course vary between different crystal structures. However, as a typical PVD process for nitrides is performed at a third of the melting temperature [66], the vibrational contributions are at least not dominating the free energy balance.

The magnetic contributions in Eq. (4.5) can be substantial, although they are heavily dependent on the material system. In the systems covered in this thesis, none of them are magnetic and will, therefore, not be affected by these terms.

The configurational entropy $S^{\text {conf }}$ can, for a binary or quasi-binary ${ }^{1}$ solution, be reasonably approximated through a mean field approach with

$$
S^{\mathrm{conf}}=-k_{B}(x \ln (x)+(1-x) \ln (1-x)),
$$

where $k_{B}$ is the Boltzmann constant. Although $S^{\text {conf }}$ is constant for a specific concentration, it will be scaled with the temperature and increase its influence. The critical temperature for when a disordered alloy phase will be favorable over an ordered phase can be approximated with Eq. (4.6).

Surprisingly often it is enough to approximate the entropy additions with zero, then the remaining important contribution comes from the ground state mixing enthalpy of each respective alloy phase. Thus, important information can be gained from the mixing enthalpy alone, which, for a random alloy at zero pressure, can be calculated from total energies calculations at the equilibrium volumes using DFT and the SQS formalism described previously.

\footnotetext{
${ }^{1} \mathrm{~A}$ quasi-binary solution is a solution with more than two components, where the composition is only varied between two components, e.g., $\mathrm{A}_{x} \mathrm{~B}_{1-x} \mathrm{~N}$, since $\mathrm{N}$ is on a separate sublattice.
} 


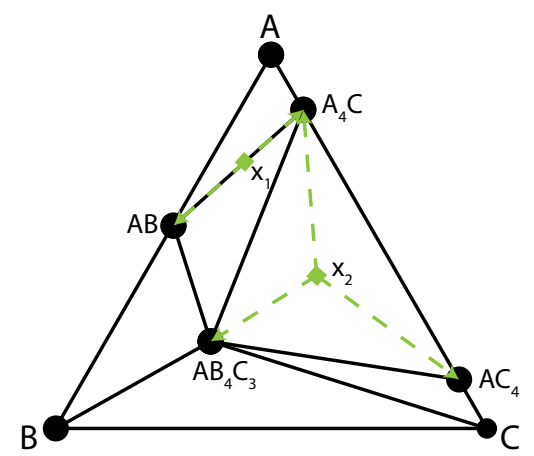

Figure 4.2. Phase diagram of a hypothetical ternary alloy composed of A, B, and C type atoms. $x_{1}$ and $x_{2}$ represent two unstable compositions that will decompose according to the green dashed arrows.

In addition to the bulk thermodynamics, the surface energies of each particular phase are also relevant when predicting the structures of thin films. Nonetheless, equally important is a direct consideration of the growth kinetics, as will be discussed later in Chapter 7.1.

\subsection{Phase stability of multicomponent alloys}

The previous section described the relatively simple phase stability of a binary or quasi-binary alloy. Although phase stability criteria is straightforwardly generalized to multicomponent systems, it becomes increasingly complicated the more components there are to compute and a visualize. Ternary alloy phase diagrams are typically visualized like in Fig. 4.2. This figure shows a ternary phase diagram for an alloy consisting of $\mathrm{A}, \mathrm{B}$, and $\mathrm{C}$ atomic species at a specific temperature. Stable structures are marked with black dots, and $x_{1}$ and $x_{2}$ mark two unstable phases. The stable phases make up a convex hull, where phases above the hull will decompose into one or a mixture of stable phases. In what way it decomposes is determined by the features of the phase diagram.

The simplest example is an unstable phase at a stable composition. This phase will restructure itself until it eventually ends up in the stable phase at that composition. A phase on one of the phase lines of the phase diagram in between two stable compositions, e.g. $x_{1}$, will decompose towards those stable compositions at the end points of the line. For a composition inside one of the triangular fields of the phase diagram, such as $x_{2}$, will decompose into a combination of the stable phases which make up the corners of the triangle.

The phase lines in the phase diagram, i.e. the convex hull construction, requires finding the minimum total energy $E_{\mathrm{tot}}^{\min }$ of an elemental composition of $N^{A}, N^{B}$, and $N^{C}$. This is calculated, in line with Ref. [67], by

$$
E_{\text {tot }}^{\min }=\min \left\{E_{\text {tot }}\left(N^{A}, N^{B}, N^{C}\right)\right\} \text {, }
$$


The set of total energies consist of the total energies that fulfill

$$
E_{\text {tot }}\left(N^{A}, N^{B}, N^{C}\right)=\sum_{i}^{n} x_{i} E_{i}
$$

where $x_{i}$ and $E_{i}$ are the amount and energy of the $i$ :th stable compound, where the restrictions

$$
x_{i} \geq 0, \quad \sum_{i}^{n} x_{i}^{A}=N^{A}, \quad \sum_{i}^{n} x_{i}^{B}=N^{B}, \text { and } \quad \sum_{i}^{n} x_{i}^{C}=N^{C},
$$

apply. The restrictions require that no compound composition can be negative and the sum of $\mathrm{A}, \mathrm{B}$, and $\mathrm{C}$ type atoms need to be constant. An unknown new structure of interest $\mathrm{A}_{N^{A}} \mathrm{~B}_{N^{B}} \mathrm{C}_{N^{C}}$ can then be compared to $E_{\text {tot }}^{\min }$ to determine the stability of this structure through the difference

$$
\Delta E\left(A_{N^{A}} B_{N^{B}} C_{N^{C}}\right)=E\left(A_{N^{A}} B_{N^{B}} C_{N^{C}}\right)-E_{\mathrm{tot}}^{\min }\left(N^{A}, N^{B}, N^{C}\right) .
$$

The structure will decompose if $\Delta E$ is positive, and if it is negative, the structure is stable and will be a part of the new hull.

\subsection{Phase stability of $\mathrm{TM}_{0.5} \mathrm{Zn}_{0.5} \mathrm{~N}$ alloys}

The piezoelectric properties of wurtzite $\mathrm{TM}_{0.5} \mathrm{Zn}_{0.5} \mathrm{~N}$ alloys are highly interesting, as is discussed in the previous chapter. Their phase stability is, however, not known. In Paper $\mathrm{V}$ we determined that the disordered wurtzite phase is lowest in energy of the investigated disordered rocksalt, zincblende, layered-hexagonal, and wurtzite phases. However, there could be other phases that are more stable or it could be more energetically favorable to decompose. In order to determine the stability of the $\mathrm{TM}_{0.5} \mathrm{Zn}_{0.5} \mathrm{~N}$ alloys, we need the convex hull to compare it to.

The aim of Paper VI is to investigate the phase stability of the wurtzite structure in the mostly unexplored $\mathrm{TM}_{x} \mathrm{Zn}_{y} \mathrm{~N}_{z}$ phase space using a high-throughput approach. The test structures were created by modifying known structures with two cations and one anion, where the anion is replaced by $\mathrm{N}$ and the cations were replaced by a permutation of the TM and $\mathrm{Zn}$ atoms. This resulted in 8370 ternary test structures for each phase diagram.

The phase diagram of TiZnN and $\mathrm{ZrZnN}$ are presented in Fig. 4.3 (a) and (b). These phase diagrams both show that the $\mathrm{TMZnN}_{2}$ composition is stable. However, the stable phase differs between the systems.

It is important to point out that phases with $\mathrm{TMZnN}_{2}$ compositions are only stable when the $\mathrm{N}_{2}$ energy is shifted by a correction term of $0.7485 \mathrm{eV}$ per $\mathrm{N}_{2}$ molecule. This corrections is needed because of the higher inaccuracy of GGA treating molecules with respect to solids. However, $\mathrm{Zn}_{3} \mathrm{~N}_{2}$ is also unstable with respect to GGA $\mathrm{N}_{2}$ without the correction, and is known to be stable and have been synthesized experimentally [68]. Therefore, the correction term is important. We have used the same correction energy used by the Materials Project, which is 
(a) Ti-Zn-N

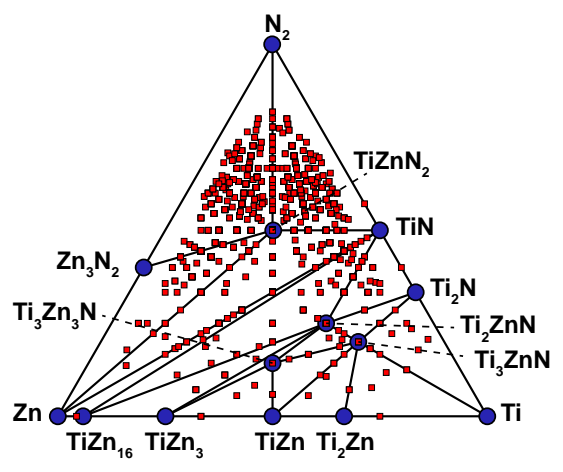

(b) Zr-Zn-N

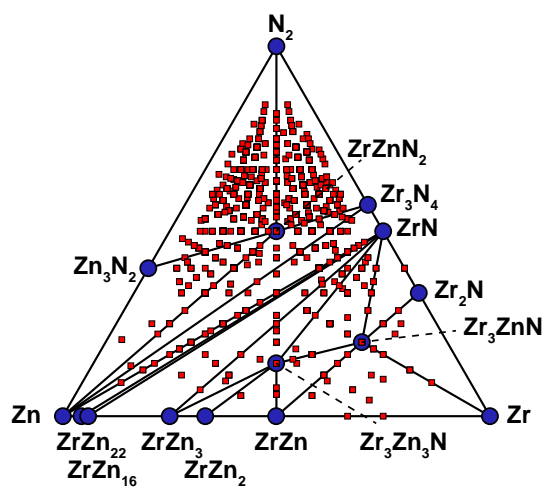

Figure 4.3. Ternary phase diagram of (a) Ti-Zn-N and (b) Zr-Zn-N. Orange circles mark stable compositions and red squares mark unstable structures.

(a) Ordered TiZnN $N_{2}$ wurtzite superstructure

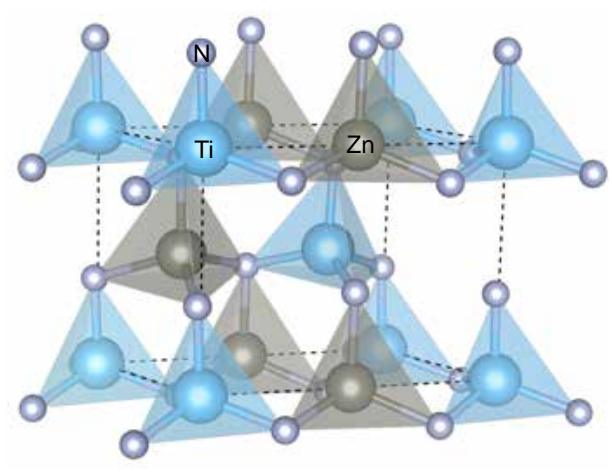

(b) Layered $\mathrm{ZrZnN}_{2}$

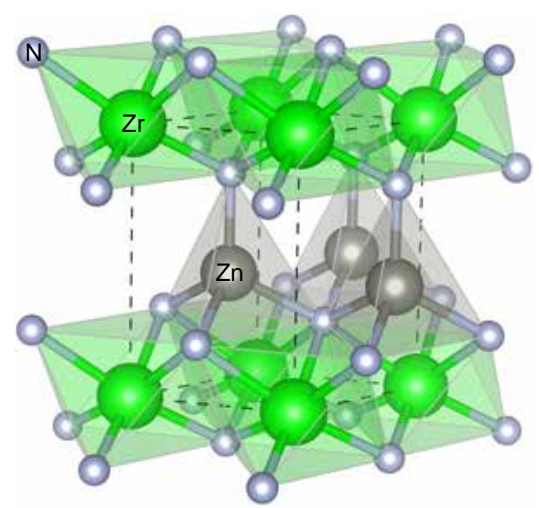

Figure 4.4. The (a) $\mathrm{TiZnN}_{2}$ and (b) $\mathrm{ZrZnN}_{2}$ crystal structures predicted to be stable. 
acquired based on the method by Wang et al. [69]. Without correction the lowest energy structure for $\mathrm{TiZnN}_{2}$ is $15 \mathrm{meV}$ /atom above the hull.

The stable phase in $\mathrm{TiZnN}_{2}$ is an ordered wurtzite structure with alternating $\mathrm{Ti}$ and $\mathrm{Zn}$ atoms in every $c$-layer, see Fig. 4.4 (a). The disordered wurtzite structure is $35 \mathrm{meV} /$ atom above the hull, and between these structures there are other ordered wurtzite structures with a slight difference in the ordering. Here, it is worth to point out that this is at $0 \mathrm{~K}$. For higher temperatures the entropy part of Gibb's free energy promotes disordered structures. However, higher temperature and low pressure also promotes the $\mathrm{N}_{2}$ gas. Thus, when designing a possible synthesis route this needs to be taken in to account. The disordered phase should, however, be energetically favorable at $1170 \mathrm{~K}$, according to a mean-field approach. This is relatively low temperature for these materials. Thus, it is likely that the disordered wurtzite exist.

The stable phase in $\mathrm{ZrZnN}_{2}$ is, however, not the wurtzite phase. Instead, the stable phase is a layered structure with alternating tetrahedric layers of $\mathrm{ZnN}$ and octahedric layers of ZrN, see Fig. 4.4 (b). Thus, the disordered wurtzite structure is much higher in energy above the hull, at $73 \mathrm{meV} /$ atom.

The reason behind the difference between $\mathrm{ZrZnN}_{2}$ and $\mathrm{TiZnN}_{2}$ is the difference in atomic volume between $\mathrm{Ti}$ and $\mathrm{Zr}$. Zr is larger than $\mathrm{Ti}$ and, therefore, the sides of the $\mathrm{ZrN}$ octahedra lines up well with the base of the $\mathrm{ZnN}$ tetrahedra, in contrast to the TiN octahedra. 


\section{CHAPTER 5}

\section{Thin film deposition}

Most of the materials investigated in the thesis are intended to be used in thin film form, understanding the basics of the synthesis is therefore critical. The material synthesis in Papers II, III, and IV, were all performed using a PVD technique, specifically magnetically unbalanced direct current reactive magnetron sputtering. The focus of this chapter is, therefore, on this technique and its modifications.

\section{$5.1 \quad$ Sputtering}

A basic sputter deposition system reactor, see Fig. 5.1, consist of a vacuum chamber, in which there is a target (source of material for the film), a substrate on which the film is grown, a plasma and a sputtering gas, e.g., Ar.

An electric field at the target is created when a negative voltage, in this case a direct current voltage, is applied to it. This electric field converts the gas to a plasma and cause the ions and electrons present in the chamber to move. The electrons are repelled by the negatively charged target, and if the field is strong enough they can cause the sputtering gas to ionize. The positively charged sputtering gas will then be attracted to the target, and sputtering will begin.

When the sputtering ions collide with the target they have enough energy to kick atoms out of the target by transferring its kinetic energy to them. Generally, neutrally charged target atoms are ejected from the surface, however, other particles, e.g. electrons, reflected ions, can also be ejected. ${ }^{1}$ Although there is a spread of ejected target atoms, the majority of the ejected target atoms will travel

\footnotetext{
${ }^{1}$ Also ionized target atoms can be ejected, although specific methods, such as high power impulse magnetron sputtering (HiPIMS) or arc evaporation, are needed in order to obtain a substantial amount of them.
} 


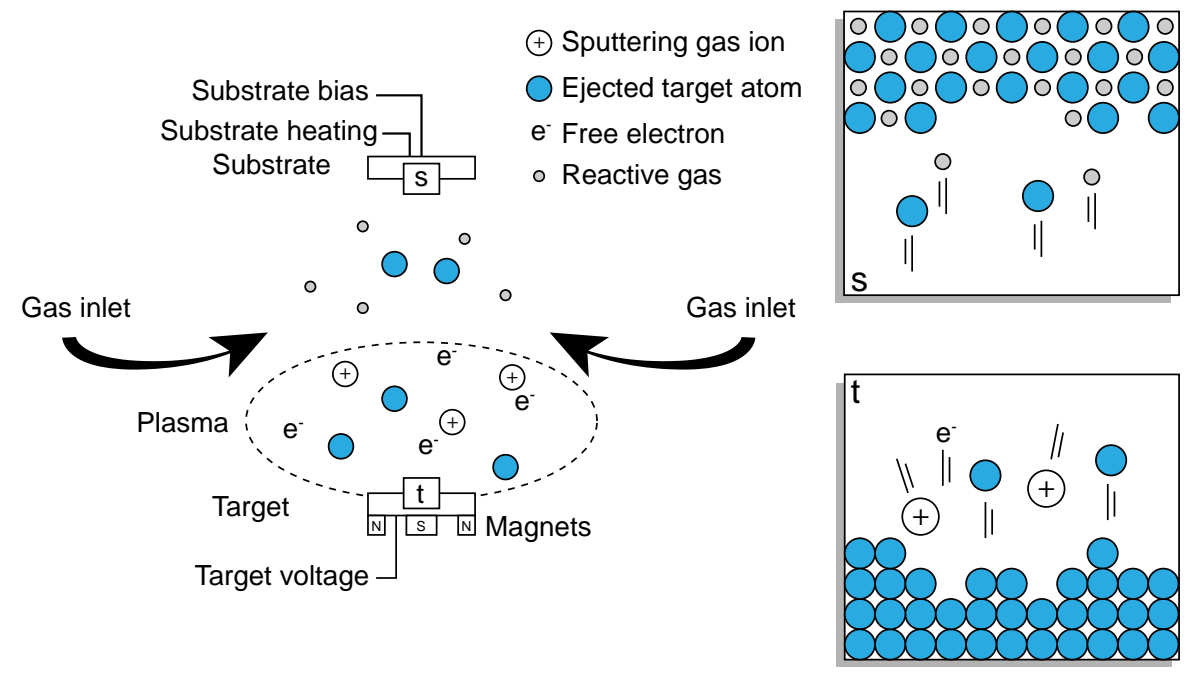

Figure 5.1. Schematic overview of a reactive sputtering deposition system, including atomic scale magnifications of the target and substrate surfaces.

straight out from the target towards the substrate. As the target atoms are traveling through the plasma, there is chance that they can become ionized. If they are, their trajectory can be altered by the magnetic field resulting in them ending up elsewhere in the reactor, e.g., on the chamber walls or the substrate holder.

The secondary electrons, ejected from the target, will help to sustain the glow discharge by ionizing additional atoms in the sputtering gas that in turn generate additional secondary electrons. When enough secondary electrons and ionized sputtering atoms are generated, the glow discharge is continuous and the gas will start to glow with a, for the target material and gas specific, characteristic color.

\subsection{Magnetron setup}

Magnetrons are used to keep the free electrons close to the target for a longer time, so that they can aid in the ionization process of the sputtering gas. Without the magnetrons and their magnetic field, the electrons will dissipate before they ionize the sputtering gas atoms and, e.g., end up on the grounded chamber walls. Effectively, a lower gas pressure is needed by using magnetrons.

The shape of the magnetic field can be modified by the magnetron setup. There are three types of setups: balanced, unbalanced type I, and unbalanced type II. In the balanced setup, the inner and outer, with opposite polarization, are equal in strength. In contrast, in the unbalanced setup the inner and outer magnets have different strengths; the type I has a stronger inner magnet and the type II has a stronger outer magnets. Type II unbalanced magnetrons are used in the reactor used to synthesize the films in Papers II, III, and IV. This setup is used because it extends the magnetic field closer to the substrate, thus aiding ionization close 
to the substrate.

\subsection{Reactive sputtering}

In reactive sputtering a gas, e.g. $\mathrm{N}_{2}$ or $\mathrm{O}_{2}$, is added to the process with the specific intent to react with the sputtered target atoms and become integrated in the film. The nitride alloys we studied were synthesized with $\mathrm{N}_{2}$ as the reactive gas in combination with Ar as the sputtering gas. Changing the ratio between the gases can have a large effect on the growth by, e.g, affecting the $\mathrm{N}$ incorporation in the film. For example, in Paper III we found that the crystalline quality of the films was superior in films grown with $60 \% \mathrm{~N}_{2}$ flow compared to those grown at $40 \% \mathrm{~N}_{2}$ flow. This can of course differ in other material systems. Furthermore, in some cases it might even be beneficial to use the reactive gas also as the sputtering gas.

\subsection{Substrate setup}

The substrate is connected to a heat source that manages the deposition temperature. By heating the substrate it is possible to tune the kinetics, including the surface reactivity of the substrate, and the diffusion on and in the substrate. In general, to create metastable thin films the substrate temperature needs to be low enough to avoid atom mobility within the film, i.e. bulk diffusion. A too low substrate temperature could, nonetheless, pose a problem since the film or substrate might not be reactive enough. Furthermore, if the surface diffusion is too limited by the low temperature, atoms would not be able to fill gaps in the growing film, and the resulting film would have poor crystalline quality.

One way to increase the reactivity and surface diffusion, without activating bulk diffusion, is to apply a substrate bias. With a negative bias on the substrate it will start to attract positive ions to the substrate. The resulting ion bombardment of the the film increases the mobility of atoms on the surface. This can affect the morphology of the film in many different ways, e.g., density, preferred crystal orientation, etc $[70,71]$. Ion bombardment will also increase the substrate temperature, although mostly close to the surface. Diffusion within the film will therefore still be restricted.

\subsection{Sputtering multiple materials}

An alternative way to introduce additional elements in the sputtering process, other than the reactive gas, is to modify the target setup. This can be done either by using a compound target or multiple target sources.

If the target positions are limited it is possible to use a compound target. In this way a single target can be a source of many atomic species. This does, however, limit the deposition to a single composition. Furthermore, the composition of the film is not necessarily randomly mixed. If the atomic species are very different in 
mass it is possible that the lightest species is sputtered first. Eventually the target will have a higher content of the second species and the flux will reach a steady state. It is, however, difficult to tune the plasma properties to fit both species at once.

If there are multiple target positions available in the reactor it is possible to have individual targets for each atomic species. This approach has the advantage that the flux from each target can be tuned with individual voltages, so that the same setup can be used to create different film compositions. Alternatively, they can be activated during select parts of the growth, e.g., to grow a specific buffer or caping layer.

An effect of having multiple targets is that not all of them can face the substrate directly. Instead they will likely be at at an angle to the substrate surface normal. In order to avoid inhomogeneous films the substrate can be continuously rotated. Substrate rotation can also be used to grow exotic nanostructures, for example, chiral nanospirals [72].

\subsection{Implications}

Thin film deposition techniques are, as mentioned in the beginning of this chapter, out of equilibrium processes. Therefore, it is possible to grow materials which are not thermodynamically stable, i.e. metastable materials. This opens up a huge material space of possibilities, where alloys with superior properties might be discovered. Furthermore, it challenges material analysis, since it is not possible to use phase diagrams in order to know which phase will obtained from thin film synthesis. Instead, theory becomes extra useful in order to know and understand which phases or alloys are synthesized. 


\section{CHAPTER 6}

\section{Metastable nitride alloys}

When using first principles modeling it is easy to find structures which are stable with respect to the allowed relaxations in the calculation, e.g., volume relaxation, where small volume changes only increases the total energy of the system. This could lead to an over-usage of the concept of metastability, being a more positive term than just instability, in theoretical works where several non-equilibrium phases in addition to the ground state is studied. However, for the term metastable to be useful, in particular in a pure theoretical analysis, a credible suggestion is needed about the nature of the constraint that is supposed to prevent equilibrium to be reached during synthesis and usage. This chapter covers how to use firstprinciples based theory to investigate metastability, in particular in ternary and quarternary nitride alloys.

\subsection{Ternary nitride alloys}

One of the most successful methods for synthesizing metastable materials is PVD, described in the previous chapter. Using such a technique it is possible to grow thin films practically atom by atom at low temperatures, typically about a third of their melting temperature, far away from equilibrium where diffusion is limited by kinetic barriers. Phase diagrams which describe equilibrium phases are, therefore, insufficient when trying to predict the resulting material from this type of synthesis. Instead, as discussed by Holleck [66], it is preferable to synthesize solid solutions with a local compositions close to the global composition of the incoming atom flux. These can, in contrast to complex ordered structures, tolerate high levels of point defects, and in particular phase separation. Furthermore, important predictions can be made by directly investigating the mixing free energy curves of the relevant phases in a material system of interest. The mixing free energies of 

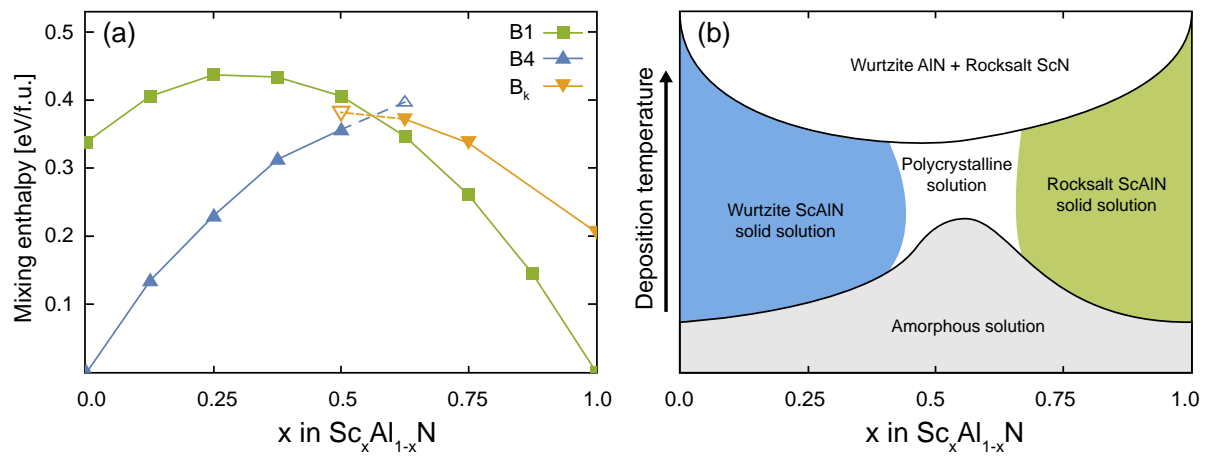

Figure 6.1. (a) Mixing enthalpies of rocksalt (B1), layered-hexagonal $\left(\mathrm{B}_{k}\right)$, and wurtzite (B4) $\mathrm{Sc}_{x} \mathrm{Al}_{1-x} \mathrm{~N}$. Adapted from Ref. [73]. (b) Phase fields schematic of film composition from different deposition temperatures during PVD growth. Adapted from Ref. [66]

disordered solid solutions can, from $0 \mathrm{~K}$ calculations, be approximated with the mixing enthalpies of an ideal random alloy, as discussed in Sec. 4.1.1.

The metastable $\mathrm{Sc}_{x} \mathrm{Al}_{1-x} \mathrm{~N}$ material system has been experimentally synthesized by many groups [5, 6, 11, 14], and is now a well known material. It is, therefore, a suitable reference system for the similar alloys investigated in Papers II, III, and V.

Ternary nitride alloys, such as $\mathrm{Sc}_{x} \mathrm{Al}_{1-x} \mathrm{~N}$, are often described by the pseudobinary mixing enthalpies, where the $\mathrm{N}$ content is constant and only the $\mathrm{Sc}$ and $\mathrm{Al}$ content is varied with respect to $x$ in $\mathrm{Sc}_{x} \mathrm{Al}_{1-x} \mathrm{~N}$. The mixing enthalpies for rocksalt, wurtzite, and layered-hexagonal structured $\mathrm{Sc}_{x} \mathrm{Al}_{1-x} \mathrm{~N}$ alloys, calculated by Höglund et al. [73], are presented in Fig. 6.1 (a). Qualitatively, the higher the mixing enthalpy, the more difficult it is to counteract the thermodynamic driving force towards phase separation. The mixing enthalpy for the wurtzite phase reaches it maximum of about $0.35 \mathrm{eV} /$ f.u. near $x=0.5$, after which the rocksalt phase is lowest in energy.

These mixing enthalpies can be used to create a schematic of the PVD phase fields diagram in line with the approach suggested by Holleck, see Fig. 6.1 (b). The phase fields diagram for $\mathrm{Sc}_{x} \mathrm{Al}_{1-x} \mathrm{~N}$ has five different phase fields. At low deposition temperatures there is not enough energy for the atoms to have time to find a preferred crystal position before more atoms are added. The result will be an amorphous solution, probably with a high level of porosity, and in most cases they have no practical value. At higher temperatures, it will be possible to create dense films consisting of a solid solution of the phase with the lowest energy for each particular composition, wurtzite $\mathrm{Sc}_{x} \mathrm{Al}_{1-x} \mathrm{~N}$ at low $x$ and rocksalt $\mathrm{Sc}_{x} \mathrm{Al}_{1-x} \mathrm{~N}$ at high $x$. Closest to the transition point between the wurtzite and rocksalt phases, both phases will be competitive and the synthesis will likely result in a polycrystalline mixture of them both. Finally, at even higher deposition temperatures, there will be enough thermal energy available for diffusion to drive $\mathrm{Sc}_{x} \mathrm{Al}_{1-x} \mathrm{~N}$ toward equilibrium by decomposing into a mixture of wurtzite AlN 

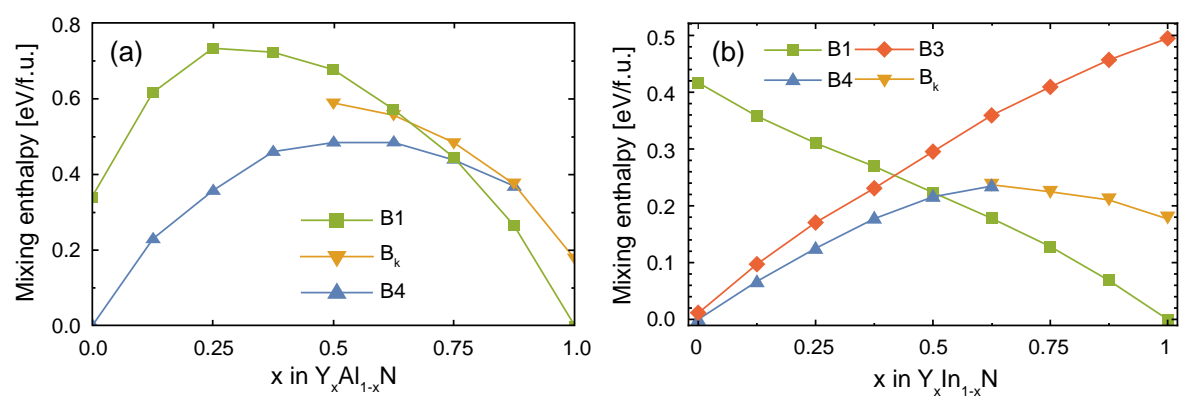

Figure 6.2. (a) Mixing enthalpies of rocksalt (B1), layered-hexagonal $\left(\mathrm{B}_{k}\right)$, and wurtzite (B4) $\mathrm{Y}_{x} \mathrm{Al}_{1-x} \mathrm{~N}$. Remodeled from Paper II. (b) Mixing enthalpies of rocksalt (B1), zincblende (B3), layered-hexagonal $\left(\mathrm{B}_{k}\right)$, and wurtzite (B4) $\mathrm{Y}_{x} \operatorname{In}_{1-x} \mathrm{~N}$. Adapted from Paper III.

and rocksalt $\mathrm{ScN}$ equilibrium phases.

Note that the regimes in the phase fields diagram are not exact. They depend heavily on the possibility for diffusion during synthesis, which in case of surface diffusion is not only a function of temperature but also energetic ion bombardment, growth rate, etc. For example, switching substrates can shift the limits of the regimes, for $\mathrm{Sc}_{x} \mathrm{Al}_{1-x} \mathrm{~N}$ this has been shown by Höglund et al. [73]. Despite its limitations, the phase fields diagram and its underlying analysis based on mixing enthalpies is very useful when designing new metastable alloys.

The phase field diagram of $\mathrm{Sc}_{x} \mathrm{Al}_{1-x} \mathrm{~N}$ in Fig. 6.1 (b) is very similar to what has been observed experimentally. The wurtzite phase has been successfully synthesized up to $x=0.5[6,11,73]$. However, the piezoelectric response deteriorates rapidly at $x$ larger than $\sim 0.45$, where a part of the film is probably consisting of a polycrystalline mixture of phases even if this is not explicitly possible to detect with x-ray diffraction. The cubic rocksalt phase has has also been reported. With a wurtzite seed layer it has been synthesized in the range $0.72<x<1.00$ [73], but with a cubic template seed layer it can be stabilized in an even wider range $0.40<x<1.00[74]$.

\subsection{1 $\quad \mathrm{Y}_{x} \mathrm{Al}_{1-x} \mathrm{~N}$}

The calculated mixing enthalpies for $\mathrm{Y}_{x} \mathrm{Al}_{1-x} \mathrm{~N}$, see Fig. 6.2 (a), is qualitatively similar to those of $\mathrm{Sc}_{x} \mathrm{Al}_{1-x} \mathrm{~N}$. However, there are distinct differences with respect to both the wurtzite-to-rocksalt crossing point, and the maximum wurtzite mixing enthalpy.

The wurtzite phase is in $\mathrm{Y}_{x} \mathrm{Al}_{1-x} \mathrm{~N}$ lowest in energy all the way up to $x=0.75$, which could indicate the possibility to stabilize the wurtzite phase to higher $\mathrm{Y}$ content as compared to $\mathrm{Sc}$ contents in $\mathrm{Sc}_{x} \mathrm{Al}_{1-x} \mathrm{~N}$ as the competing rocksalt phase is of lower concern. However, the magnitude of the positive mixing enthalpy is substantially higher in $\mathrm{Y}_{x} \mathrm{Al}_{1-x} \mathrm{~N}$ than $\mathrm{Sc}_{x} \mathrm{Al}_{1-x} \mathrm{~N}$ meaning it will be more difficult to avoid phase separation. The mixing enthalpies of the $\mathrm{Y}_{x} \mathrm{Al}_{1-x} \mathrm{~N}$ wurtzite phase 
is near $0.35 \mathrm{eV} / \mathrm{f} . \mathrm{u}$., the mixing enthalpy of the wurtzite phase in $\mathrm{Sc}_{x} \mathrm{Al}_{1-x} \mathrm{~N}$ at the maximum Sc content reported, already at $x=0.25$. Synthesizing good quality $\mathrm{Y}_{x} \mathrm{Al}_{1-x} \mathrm{~N}$ wurtzite films at higher concentrations is, thus, predicted to be difficult.

The mixing enthalpies of rocksalt and wurtzite $\mathrm{Y}_{x} \mathrm{Al}_{1-x} \mathrm{~N}$ has also previously been calculated by Rovere et al. [75]. The effect of adding the layered-hexagonal phase, that is lower than the wurtzite phase in pure YN, is that the wurtziterocksalt crossing point is lowered from $x=0.875$ to $x=0.75$.

In Paper II we report synthesis of wurtzite $\mathrm{Y}_{x} \mathrm{Al}_{1-x} \mathrm{~N}$ films up to $x=0.22$, and recently Mayrhofer et al. [76] was able to measure the increased piezoelectric response in $\mathrm{Y}_{x} \mathrm{Al}_{1-x} \mathrm{~N}$ in the range $0<x \leq 0.116$. They report an increase from $d_{33, f}=3.2 \mathrm{pC} / \mathrm{N}$ at $x \approx 0.01$ to $3.7 \mathrm{pC} / \mathrm{N}$ at $x=0.059$, for their best $c$-axial films.

The mixing difficulty in $\mathrm{Y}_{x} \mathrm{Al}_{1-x} \mathrm{~N}$ can be attributed to the large volume difference between $\mathrm{Y}$ and $\mathrm{Al}$ atoms and consequently between the unit cell volume of layered-hexagonal YN and wurtzite AlN. The volume difference between YN and $\mathrm{AlN}$ is $60 \%$, while the difference between the corresponding phases of $\mathrm{ScN}$ and $\mathrm{AlN}$ is only $23 \%$. Because of the extremely strong de-mixing tendency in $\mathrm{Y}_{x} \mathrm{Al}_{1-x} \mathrm{~N}$, it is better to look at other material systems in order to get a good $c$-axial oriented piezoelectric nitride thin film.

\subsection{2 $\mathrm{Y}_{x} \operatorname{In}_{1-x} \mathrm{~N}$}

The unit cell volume difference between layered-hexagonal $\mathrm{YN}$ and wurtzite $\mathrm{InN}$ is much lower than both that between $\mathrm{YN}$ and $\mathrm{AlN}$ and that between $\mathrm{ScN}$ and AlN. The stability of the $\mathrm{Y}_{x} \mathrm{In}_{1-x} \mathrm{~N}$ material system is investigated in Paper III using both theory and experiments. The mixing enthalpies of $\mathrm{Y}_{x} \operatorname{In}_{1-x} \mathrm{~N}$ we report, see Fig. 6.2 (b), are qualitatively similar to those of the $\mathrm{Sc}_{x} \mathrm{Al}_{1-x} \mathrm{~N}$ system. The absolute values of the positive mixing enthalpies are, however, somewhat lower with the maximum mixing enthalpy of the wurtzite phase in $\mathrm{Y}_{x} \operatorname{In}_{1-x} \mathrm{~N}$ just above 0.2 $\mathrm{eV} /$ f.u. Furthermore, in this study we also included the cubic zincblende structure, which is very similar to the wurtzite with the difference being the stacking of the layers along the $c$-direction. In the zincblende crystal structure the layers are ABCABC stacked, and in wurtzite crystal structure they are ABAB stacked.

Experimentally, we managed to produce wurtzite $\mathrm{Y}_{x} \operatorname{In}_{1-x} \mathrm{~N}$ thin films with an $\mathrm{Y}$ content of up to $x=0.51$. However, the crystalline quality of the films starts to deteriorate after $x=0.14$ as indicated with lower intensity, broader, x-ray diffraction peaks. It should be noted that our work is a pioneering study on this alloy system and further experiments fine-tuning the process parameters are likely to be able to improve the quality of the films.

Because of the structural similarity between the wurtzite and zincblende phases, the two are very close in energy, especially in InN. Therefore, it is possible to obtain an unwanted polycrystalline mixture of them, as was observed in Paper III and also other works [77, 78]. The introduction of $\mathrm{Y}$ does, however, improve the crystalline quality substantially as the zincblende phase becomes increasingly unfavorable compared to the wurtzite phase.

Compared to AlN, the formation energy and disassociation temperature of $\mathrm{InN}$ 
is lower and, therefore, much more difficult synthesize with enough $\mathrm{N}$ to keep the 1:1 stoichiometry. This can lead to the formation of metal In droplets that was observed in Paper III and elsewhere [79]. These droplet are, however, not observed in the samples with $\mathrm{Y}$. Thus, even a small amount of $\mathrm{Y}$ is enough to help bind the $\mathrm{N}$ in the film and avoid the formation of metal droplets. Moreover, the problem with saturating the $\mathrm{N}$ content in $\mathrm{InN}$ could also be an indication that the pseudobinary mixing enthalpies might be insufficient to fully understand the role of $\mathrm{N}$ in InN-based ternary systems. Future studies of the full ternary phase diagram could reveal additional important information.

\subsection{Quarternary nitride alloys}

The possibility to synthesize metastable quarternary $\mathrm{Ti}_{x / 2} \mathrm{Mg}_{x / 2} \mathrm{Al}_{1-x} \mathrm{~N}$ alloy systems was expected building on our experience from the ternary cases discussed above and because of a recent study of the TiMgN alloy system by Alling [63]. He showed that $\mathrm{TiMgN}_{2}$ is thermodynamically stable in an $\mathrm{L}_{1}$-type superstructure ordering on the octahedrally coordinated cubic rocksalt lattice. A disordered random $\mathrm{Ti}_{0.5} \mathrm{Mg}_{0.5} \mathrm{~N}$ counterpart was found to be stable above a critical temperature of $950 \mathrm{~K}$. Moreover, because the valence electrons in this system equals an averaged $3+$ as in $\mathrm{Sc}^{3+}$ both ordered and disordered phases displayed semiconducting electronic character. $\mathrm{Ti}_{0.5} \mathrm{Mg}_{0.5} \mathrm{~N}$ was, therefore, suggested to be a possible substitution for $\mathrm{ScN}$ in alloy applications [63]. In alloys with $\mathrm{AlN}$ it is probable that the system displays the same relevant competing alloy phases (rocksalt, zincblende, layered-hexagonal, and wurtzite).

Extending the investigation to include $\mathrm{Zr}$, Hf, and $\mathrm{Ca}$ was a logical next step, because $\mathrm{Zr}$ and $\mathrm{Hf}$ are in the same group in the periodic table as $\mathrm{Ti}$, and $\mathrm{Ca}$ is in the same group as Mg. Thus, they are expected to have similar binding properties and relevant competing phases. The additional choice of Zn was attributed solely to the valence matching criteria, which resulted in Paper V.

\subsection{1 $\mathrm{TM}_{x / 2} \mathrm{Mg}_{x / 2} \mathrm{Al}_{1-x} \mathrm{~N}$ and $\mathrm{TM}_{x / 2} \mathrm{Ca}_{x / 2} \mathrm{Al}_{1-x} \mathrm{~N}(\mathrm{TM}=\mathrm{Ti}$, $\mathrm{Zr}, \mathrm{Hf}$ )}

The pseudo-binary mixing enthalpies for rocksalt, zincblende, layered-hexagonal, and wurtzite $\mathrm{Ti}_{x / 2} \mathrm{Mg}_{x / 2} \mathrm{Al}_{1-x} \mathrm{~N}$, see Fig. 6.3 (a), resembles those previously described $\left(\mathrm{Sc}_{x} \mathrm{Al}_{1-x} \mathrm{~N}, \mathrm{Y}_{x} \mathrm{Al}_{1-x} \mathrm{~N}\right.$, and $\left.\mathrm{Y}_{x} \mathrm{In}_{1-x} \mathrm{~N}\right)$. There is a similar transition from the wurtzite phase having the lowest in energy in AlN-rich composition to instead the rocksalt phase in $\mathrm{Ti}_{0.5} \mathrm{Mg}_{0.5} \mathrm{~N}$ rich compositions.

The maximum mixing enthalpy of the wurtzite phase is comparably low at $\sim 0.2$ $\mathrm{eV} /$ f.u. This relatively low mixing enthalpy is clearly lower than for $\mathrm{Sc}_{x} \mathrm{Al}_{1-x} \mathrm{~N}$ alloy compositions that have been demonstrated experimentally to be possible to grow. This indicates that the tendency for phase separation should not be a hinder for synthesizing $\mathrm{Ti}_{x / 2} \mathrm{Mg}_{x / 2} \mathrm{Al}_{1-x} \mathrm{~N}$ thin films up to $x=0.5$ through similar synthesis routes as the ternary nitrides, e.g., $\mathrm{Sc}_{x} \mathrm{Al}_{1-x} \mathrm{~N}$.

The mixing enthalpy curves of $\mathrm{Zr}_{x / 2} \mathrm{Mg}_{x / 2} \mathrm{Al}_{1-x} \mathrm{~N}$ and $\mathrm{Hf}_{x / 2} \mathrm{Mg}_{x / 2} \mathrm{Al}_{1-x} \mathrm{~N}$ are 


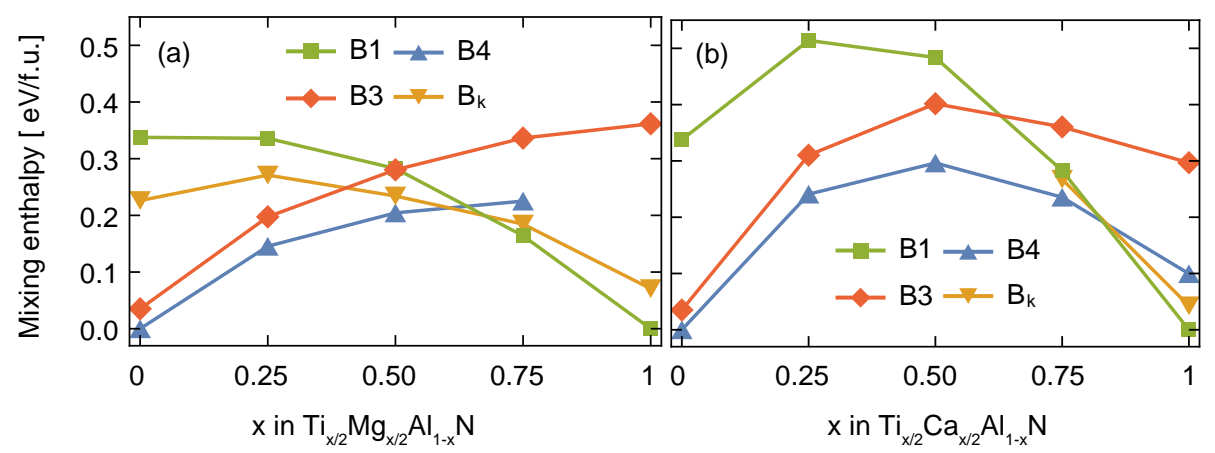

Figure 6.3. Mixing enthalpies of rocksalt (B1), layered hexagonal $\left(\mathrm{B}_{k}\right)$, and wurtzite (B4) for (a) $\mathrm{Ti}_{x / 2} \mathrm{Mg}_{x / 2} \mathrm{Al}_{1-x} \mathrm{~N}$, (b) $\mathrm{Zr}_{x / 2} \mathrm{Mg}_{x / 2} \mathrm{Al}_{1-x} \mathrm{~N}$, (c) $\mathrm{Hf}_{x / 2} \mathrm{Mg}_{x / 2} \mathrm{Al}_{1-x} \mathrm{~N}$, and (d) $\mathrm{Ti}_{x / 2} \mathrm{Ca}_{x / 2} \mathrm{Al}_{1-x} \mathrm{~N}$. Remodeled from Paper V.

qualitatively similar to those of $\mathrm{Ti}_{x / 2} \mathrm{Mg}_{x / 2} \mathrm{Al}_{1-x} \mathrm{~N}$. The mixing enthalpies are, however, quantitatively larger, with the maximum wurtzite mixing enthalpy at $\sim 0.3 \mathrm{eV} / \mathrm{f}$.u. in both alloys. The small volume difference between $\mathrm{ZrN}$ and HfN is a probable explanation to the similarity in the mixing enthalpies between their corresponding alloys.

So far there have been little experimental work on these quarternary alloys, limited to that of Yokoyama et al. [80, 81] and Minh et al. [82, 83], who have synthesized $\mathrm{TM}_{x / 2} \mathrm{Mg}_{x / 2} \mathrm{Al}_{1-x} \mathrm{~N}$ alloys and create devices with $x \leq 0.348$.

The mixing enthalpies of rocksalt, zincblende, layered-hexagonal, and wurtzite $\mathrm{Ti}_{x / 2} \mathrm{Ca}_{x / 2} \mathrm{Al}_{1-x} \mathrm{~N}$, see Fig. 6.3 (b), are more positive, displaying an increased tendency to phase separate than the corresponding $\mathrm{Mg}$ alloy. The maximum wurtzite mixing enthalpy is $\sim 0.05 \mathrm{eV} /$ f.u. higher in $\mathrm{Ti}_{x / 2} \mathrm{Ca}_{x / 2} \mathrm{Al}_{1-x} \mathrm{~N}$. The cubic rocksalt phase is much more influenced by the exchange of Ca for $\mathrm{Mg}$. The mixing enthalpy of the cubic phase is much higher than is observed in any of the other investigated alloys. This effectively shifts the crossing point between the wurtzite and cubic phases to higher $x$ just after $x=0.75$.

The Zr and Hf mixing enthalpies with $\mathrm{Ca}$ are not included in Paper V, because they are expected to exhibit a similar trend as in the $\mathrm{Mg}$ alloys, where the mixing enthalpies are slightly higher in the $\mathrm{Zr}$ and $\mathrm{Hf}$ alloys as compared to their $\mathrm{Ti}$ counterparts.

The $\mathrm{TM}_{x / 2} \mathrm{Ca}_{x / 2} \mathrm{Al}_{1-x} \mathrm{~N}$ ( $\mathrm{TM}=\mathrm{Ti}, \mathrm{Zr}$, and Hf) have thus far not been synthesized. However, since the $\mathrm{TM}_{x / 2} \mathrm{Mg}_{x / 2} \mathrm{Al}_{1-x} \mathrm{~N}$ alloys have been synthesized, it is likely possible to synthesize the $\mathrm{TM}_{x / 2} \mathrm{Ca}_{x / 2} \mathrm{Al}_{1-x} \mathrm{~N}$ alloys with a similar approach. It is, however, not known wether the limiting factor for wurtzite alloy formation is the competing rocksalt phase, or if it is phase separation induced already during growth. This will decide if the Ca-containing systems will be possible to stabilize at higher $x$ than the Mg cases, or if the opposite is true. 


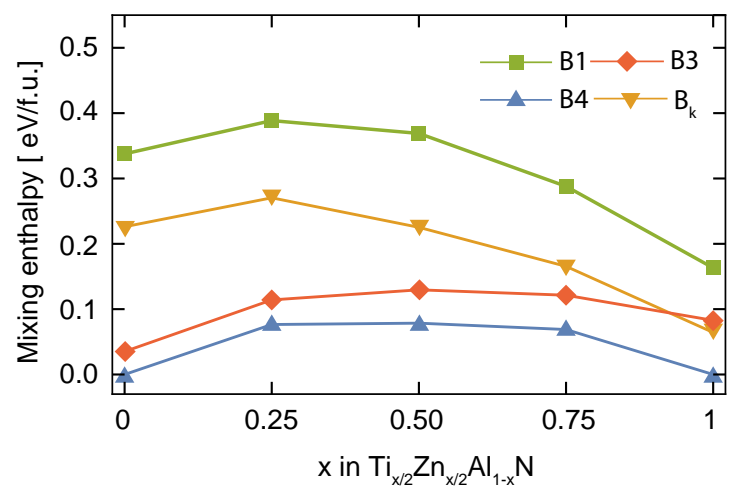

Figure 6.4. Mixing enthalpies of rocksalt (B1), zincblende (B3), layered hexagonal $\left(\mathrm{B}_{k}\right)$, and wurtzite (B4) $\mathrm{Ti}_{x / 2} \mathrm{Zn}_{x / 2} \mathrm{Al}_{1-x} \mathrm{~N}$. Remodeled from Paper V.

\subsection{2 $\mathrm{TM}_{x / 2} \mathrm{Zn}_{x / 2} \mathrm{Al}_{1-x} \mathrm{~N}(\mathrm{TM}=\mathrm{Ti}, \mathrm{Zr}, \mathrm{Hf})$}

The phase stability of $\mathrm{TM}_{x / 2} \mathrm{Zn}_{x / 2} \mathrm{Al}_{1-x} \mathrm{~N}$ is a lot more difficult to predict compared to the $\mathrm{Mg}$ and $\mathrm{Ca}$ alloys. In the $\mathrm{TM}_{x / 2} \mathrm{Mg}_{x / 2} \mathrm{Al}_{1-x} \mathrm{~N}$ alloys both of the end points, $x=0,1$, have been already investigated and the stable phases are more or less known. Because of the valence similarity between $\mathrm{Mg}$ and $\mathrm{Zn}$ the same phases should, however, be among the most relevant phases. The mixing enthalpies of $\mathrm{Ti}_{x / 2} \mathrm{Zn}_{x / 2} \mathrm{Al}_{1-x} \mathrm{~N}$, see Fig. 6.4, show that the structures are close to each other in energy. The major difference is that the order of the phases are different. In this alloy, the wurtzite phase is the most stable phase through the entire composition range. In fact there is only one visible crossing point between mixing enthalpy curves with changing composition, that from zincblende to layered-hexagonal as second lowest in mixing enthalpy.

From these mixing enthalpies alone, it is difficult to predict if this material could be metastable. The major reason is that the $\mathrm{TMZnN}_{2}$ composition is not investigated, which inspired Paper VI. The study of the full ternary TM-Zn-N phase diagrams in Paper VI does show that the ordered wurtzite $\mathrm{TiZnN}_{2}$ phase is stable, at least at $0 \mathrm{~K}$. It should, therefore, be possible to synthesize $\mathrm{Ti}_{x / 2} \mathrm{Zn}_{x / 2} \mathrm{Al}_{1-x} \mathrm{~N}$ in line with the previous cases discussed above. The $\mathrm{Zr}$ and $\mathrm{Hf}$ combinations are more difficult to predict since the wurtzite $\mathrm{ZrZnN}_{2}$ and $\mathrm{HfZnN}_{2}$ phases are not stable. The wurtzite phases are, however, close to the hull also in the Al-free limit, so at low $x$, closer to AlN the effect of the slightly less stable Zr and Hf wurtzite phases should be small and synthesis through PVD is promising.

\subsection{Strained systems}

One of the key parameters that can be used in thin film synthesis to stabilize a phase enough to make it metastable is the choice of the substrate. For example, the hexagonal tetrahedrally coordinated phase of a nitride material can be stabilized as a thin film by using a hexagonal tetrahedrally coordinated substrate that promotes 
(a)

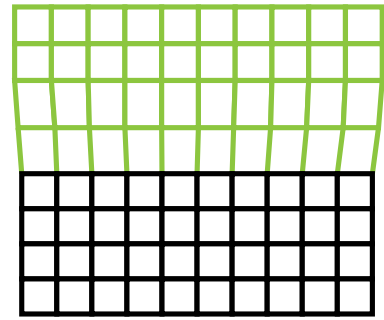

(b)

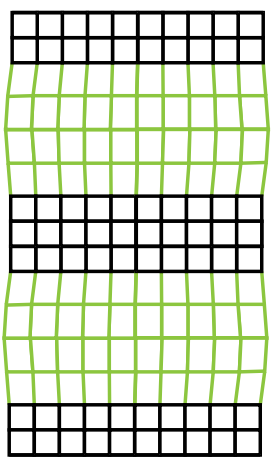

Figure 6.5. Simple illustration of (a) film under compressive strain on top of substrate, (b) layers under compressive strain in multilayer.

heteroepitaxial growth. There is, however, usually some lattice mismatch between film and substrate. This causes either tensile or compressive strain in the film. For example, a large lattice film on a small substrate, see Fig. 6.5 (a), will be subjected to compressive strain because of the lattice mismatch, creating a set of pseudomorphic layers. The effect of the lattice mismatch will decrease with the thickness of the film. The film will eventually relax the strain, through for example introduction of edge dislocations, to reach its equilibrium lattice configurations.

If the pseudomorphic part of the film possess interesting material properties, then it would be useful to try stabilizing it throughout the film. The strain on the film can be reintroduced by adding another template layer, stable in the desired structure, and in our example with smaller in-plane lattice constant. This can then be continued with more and more alternating layers, creating a multilayer film, see Fig. 6.5.

In Paper IV we observed that it is possible to stabilize the wurtzite $\mathrm{Sc}_{x} \mathrm{Al}_{1-x} \mathrm{~N}$ phase by introducing strain effects in a multilayered super structure. When comparing different layerings of $\mathrm{Sc}_{x} \mathrm{Al}_{1-x} \mathrm{~N}$ on $\mathrm{In}_{y} \mathrm{Al}_{1-y} \mathrm{~N}(\mathrm{x}=0.2,0.4 ; \mathrm{y}=0.2-0.72)$, we observed that $\mathrm{Sc}_{x} \mathrm{Al}_{1-x} \mathrm{~N}$ under tensile biaxial stress from the In-rich $\operatorname{In}_{y} \mathrm{Al}_{1-y} \mathrm{~N}$ layers have the best crystalline quality.

Why tensile stresses improve the $\mathrm{Sc}_{x} \mathrm{Al}_{1-x} \mathrm{~N}$ crystalline quality can be explained by the energetics of the rocksalt, zincblende, layered-hexagonal and wurtzite phases under in-plane stress, see Fig. 6.6 (a) and (b). Here, the cubic rocksalt and zincblende structures are aligned so that the (111) direction correspond to the $c$-direction in for the hexagonal case and represented with their counterparts to the in-plane hexagonal lattice constants. In ScN (Fig. 6.6 (a)) and AlN (Fig. 6.6 (b)) the rocksalt and zincblende structures become less energetically favored with increasing in-plane lattice constants, as compared to the wurtzite and layeredhexagonal phases. Moreover, the layered-hexagonal phase becomes more energetically favorable in both $\mathrm{ScN}$ and AlN compared to wurtzite as the in-plane lattice becomes very large. The wurtzite phase of AlN becomes indistinguishable after $\sim 3.4 \AA$. 

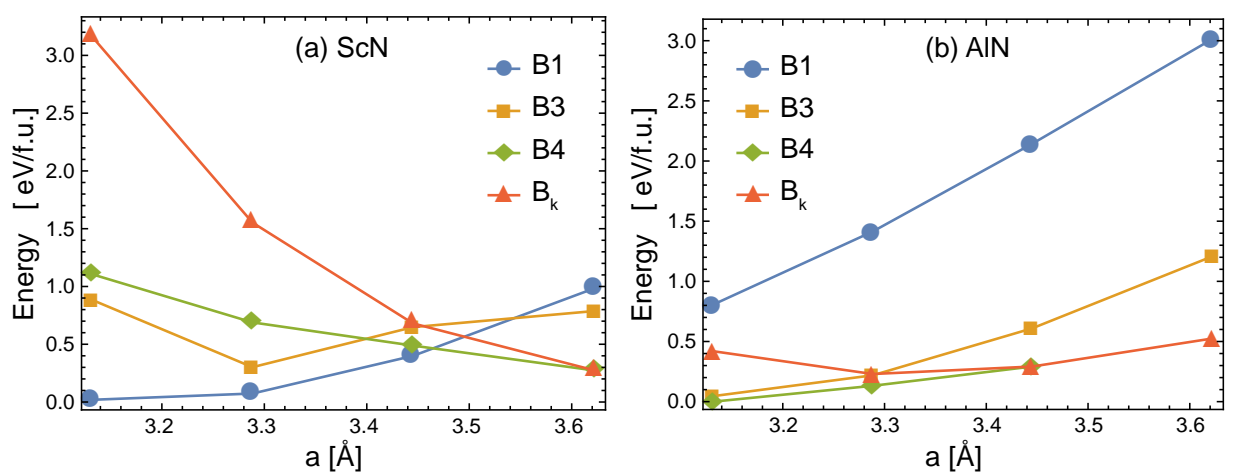

Figure 6.6. Energy of the rocksalt (B1), zincblende (B3), layered hexagonal $\left(\mathrm{B}_{k}\right)$, and wurtzite (B4) phases with respect to in-plane lattice parameter $a$ in the range $a_{\mathrm{AlN}} \leq a \leq$ $a_{\text {InN }}$ for (a) ScN and (b) AlN. For the cubic B1 and B3 structures the (111) direction is considered as the $c$-direction and $a$ is the corresponding hexagonal in-plane lattice parameter for that $c$.

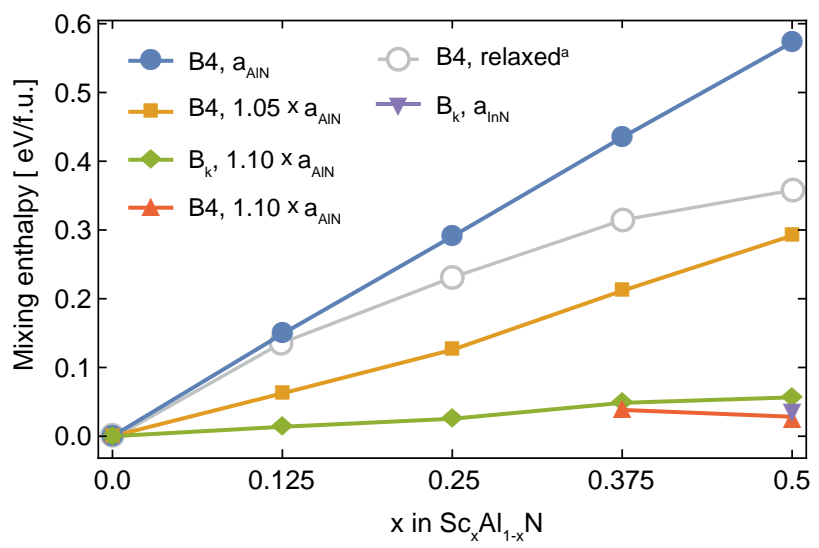

Figure 6.7. Mixing enthalpies of wurtzite (B4) and layered hexagonal $\left(\mathrm{B}_{k}\right) \mathrm{Sc}_{x} \mathrm{Al}_{1-x} \mathrm{~N}$ at fixed in-plane lattice parameters. ${ }^{\mathrm{a}}$ Is the relaxed mixing enthalpy from Ref. [73]. Remodeled from Paper IV. 
Because of the strain in this material, we calculate the mixing enthalpy with respect to the strained $\mathrm{AlN}$ and $\mathrm{ScN}$ constituents according to

$$
\Delta H_{\text {mix }}^{a}=E\left(S c_{x} A l_{1-x} N\right)^{a}-\left(x E_{S c N}^{a}+(1-x) E_{A l N}^{a}\right),
$$

where the energies are calculated at the same $a$. Through this relation we observe that the mixing is heavily influenced by the in-plane strain, see Fig.6.7, where the wurtzite phase of a fully relaxed structure is compared to a set of specific in-plane lattice-parameters $\left(a=a_{A l N}, 1.05 \times a_{A l N}, 1.10 \times a_{A l N}\right.$, and $\left.a_{I n N}\right)$. For the most restricted in-plane parameter, $a_{A l N}$, the wurtzite mixing enthalpy is increased, compared to the fully relaxed case, by the added compressive strain. For the other cases where the in-plane lattice parameter is larger, the mixing enthalpies are much lower. An alternative but equivalent formulation to the stabilization of the alloy is that the competing, phase separated case(and in particular rocksalt $\mathrm{ScN}$ ), is destabilized. However, the increased in-plane will eventually promote the layered-hexagonal phase at lower concentrations of Sc. The wurtzite phase, when the in-plane lattice parameter is $1.18 \times a_{A l N}$, is not stable until $x=0.375$.

There are two main limitation to the predictions from the approach with the strained mixing enthalpy. First, it does not take into account the loss of strain through the introduction of an array of edge dislocations as the layer thickness is increased. If the layer thickness is too large, a strain gradient through the $\mathrm{Sc}_{x} \mathrm{Al}_{1-x} \mathrm{~N}$ layers could be expected. This approach will likely limit the effect of the epitaxial stabilization. Second, this does not take into account interface atomic template effects. It is possible that the interface further limits phase separation away from the wurtzite phase.

Multilayer structures, such as the $\mathrm{Sc}_{x} \mathrm{Al}_{1-x} \mathrm{~N} / \mathrm{In}_{y} \mathrm{Al}_{1-y} \mathrm{~N}$, can also exhibit piezoelectric response. However, the magnitude is difficult to predict. Although the piezoelectric response in the $\mathrm{Sc}_{x} \mathrm{Al}_{1-x} \mathrm{~N}$ layers is high, it is low in the $\mathrm{In}_{y} \mathrm{Al}_{1-y} \mathrm{~N}$ layers. The layer thicknesses should, therefore, be optimized in such a way that the ratio of $\mathrm{Sc}_{x} \mathrm{Al}_{1-x} \mathrm{~N}$ to $\mathrm{In}_{y} \mathrm{Al}_{1-y} \mathrm{~N}$ is as high as possible without loosing too much of the stabilization effects of the wurtzite structure at high Sc content. This would be an interesting topic to look deeper into in future studies. 


\section{CHAPTER 7}

\section{Kinetics in disordered alloys}

Atomic kinetics, and diffusion in particular, is one of the most decisive forces during growth of metastable thin films. A theoretical study of its key features could therefore help in the design of growth parameters before experiments. This chapter is outlined to cover the basics of diffusion and how to model diffusion, in general, and on alloy surfaces in particular. In addition, $\operatorname{TiN}$ and $\mathrm{Ti}_{0.5} \mathrm{Al}_{0.5} \mathrm{~N}$ surface diffusion is described.

\subsection{Modeling diffusion}

There are many ways to model atomic diffusion, each with its own pros and cons. Of these, quantum molecular dynamics (QMD) has potential to be the most accurate. The basic approach of this method is to calculate the forces between the atoms in a system with quantum mechanical accuracy, then evolve the system in time using these forces and update the atomic positions. The downside to this approach is the huge computational resources that are required to maintain this kind of accuracy. Thus, in practice QMD is not necessarily very accurate due to limitations in treatable supercell size to systems with a few hundreds of atoms and the simulation time to a couple of ns. The frequencies of most diffusion events at low temperatures (compared to the melting temperature of nitrides ${ }^{1}$ ) are so low that they hardly can be captured within this time. The accuracy of the calculations can, therefore, be reduced by lack of significant statistics.

With classical molecular dynamics (CMD), it is possible to simulate much larger systems ( $\sim 10^{6}$ atoms and more) for longer periods of time than QMD (at least a few $\mu \mathrm{s}$ ). Of course, the accuracy of this method relies on the precision of the

\footnotetext{
${ }^{1}$ Melting temperature of $\mathrm{TiN}$ is $\sim 3200 \mathrm{~K}$.
} 
(a)

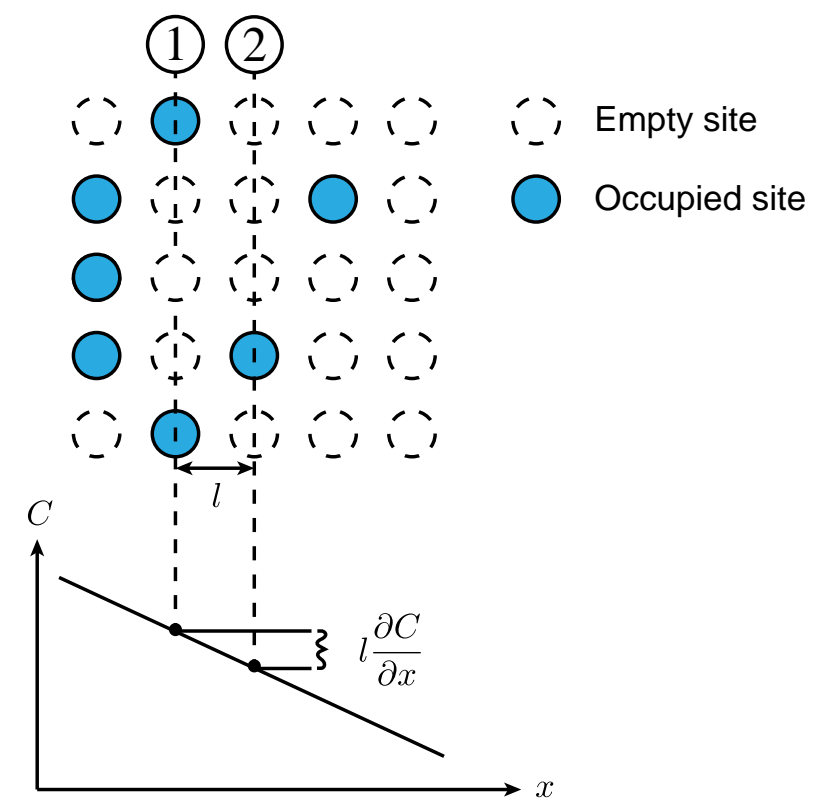

Figure 7.1. (a) A simple example of adatoms and empty sites on a cubic surface. (b) The concentration profile in the $x$-direction. Adapted from Ref. [64].

force field parametrization, and increasing the accuracy will reduce the obtainable simulation time.

However, both QMD and CMD are impractical when the qualitative effects of alloying is of interest. Finding the correct parameters for each CMD force field or running enough QMD runs to find specific transitions require massive computational resources. A much more effective approach is to use transition state theory (TST) connected to DFT, and focus on how alloying affects the potential energy barriers of the transitions. It might seem like an inaccurate method to use a static approach which does not include any of the diffusion dynamics but the dominating parameter for diffusion at low temperatures is the potential energy barrier between sites. Moreover, one could argue that the effect of alloying on the vibrational degrees of freedoms is much less than its effect on the transition barrier when considering dilute cases (see Paper VIII). The knowledge obtained by DFT-TST can also be taken one step further to simulate growth by using the parameters together with kinetic Monte Carlo (KMC) algorithms.

Regardless of the approach used to study kinetics in disordered alloys, it is important to understand the relations between the relevant parameters affecting kinetics. The primary relations are captured in Fick's two laws, which are described in the following sections. 


\subsubsection{Fick's first law}

Fick's first law describes the connection between the flux of atoms and the concentration gradient, an important relation for kinetics. To understand the relation, start by considering a surface of a cubic lattice as shown in Fig. 7.1 (a), where the diffusing atoms are distributed according to the concentration profile in (b). For a surface where no adatom migration attempt will be blocked by occupied cites, the adatom movement to the right by jumping to unoccupied lattice sites with the flow $\vec{J}_{x}$ given by

$$
\overrightarrow{J_{x}}=\Gamma_{x} n_{1}
$$

where $\Gamma_{x}$ is the number of successful jumps in the $x$-direction per unit time, $n_{1}$ is the number of adatoms per unit length in line 1 . In the opposite direction from line 2 , where there are $n_{2}$ occupied sites along line 2 , the flow of adatoms is given by

$$
\overleftarrow{J}_{x}=\Gamma_{x} n_{2}
$$

The difference between these two is the net flow in the $x$-direction,

$$
J_{x}=\vec{J}_{x}-\overleftarrow{J}_{x}=\Gamma_{x}\left(n_{1}-n_{2}\right)
$$

With $l$ as the separation between the lines, the concentration of adatoms in one line can be written as $C_{1}=n_{1} / l$ and $C_{2}=n_{2} / l$, so that $\left(n_{1}-n_{2}\right)=l\left(C_{1}-C_{2}\right)$. The change in concentration shown in Fig. 7.1 (b) can be written as $\left(C_{1}-C_{2}\right)=$ $-l(\partial C / \partial x)$, thus

$$
J_{x}=-l^{2} \Gamma_{x} \frac{\partial C}{\partial x} .
$$

Here we define the diffusivity as

$$
D=l^{2} \Gamma_{x}
$$

so that we end up with

$$
J=-D \frac{\partial C}{\partial x}
$$

known as Fick's first law, suggested by Adolf Fick in 1855 [84, 85]. Although the first law is enough for a steady-state situations, Fick's second law is needed to describe diffusion when the concentration profile is time dependent.

\subsubsection{Fick's second law}

For non-steady-state systems the concentration varies both with respect to time and distance. In order to calculate how the concentration at any point varies with time, we consider a narrow band on the surface with width $w$ and thickness $\delta x$, see Fig. 7.2 (a). The number of adatoms which diffuse into this band is given by

$$
\delta n_{1}=J_{1} w \delta t
$$

and the number of adatoms diffusing out of the band is given by

$$
\delta n_{2}=J_{2} w \delta t
$$


(a)

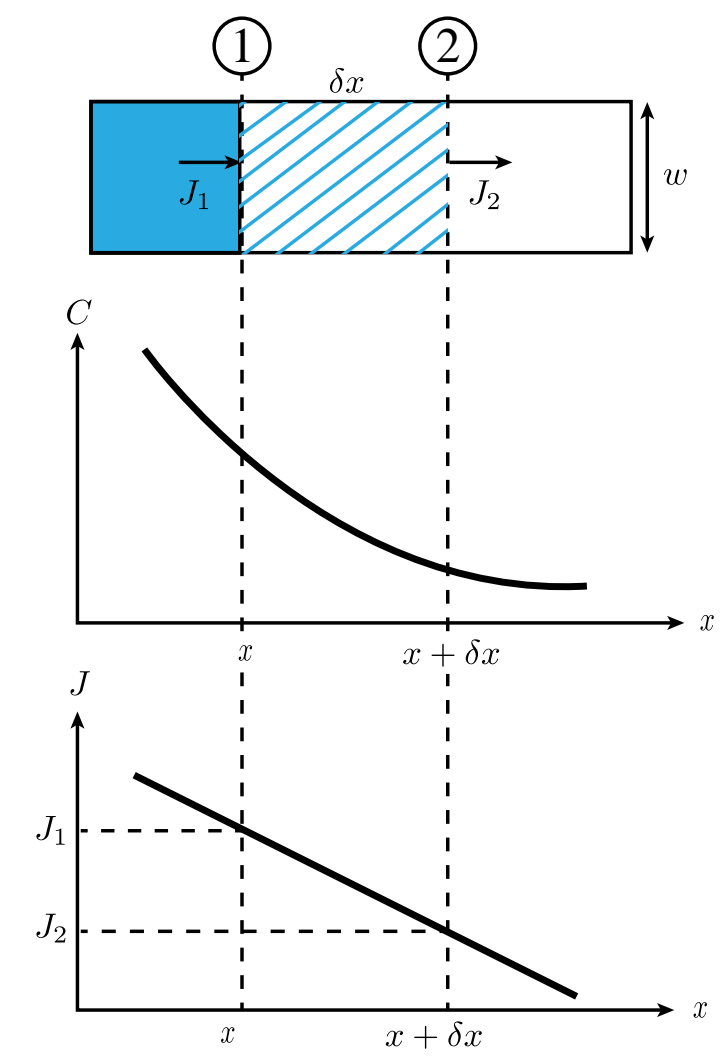

Figure 7.2. Derivation of Fick's second law. (a) A narrow band on the surface with width $w$ which adatoms enter with the flux $J_{1}$ and leaves with the flux $J_{2}$. (b) Concentration profile with respect to distance along the $x$-direction. (c) Corresponding adatom flows. Adapted from Ref. [64].

The concentration of atoms within the band will change according to

$$
\delta C=\frac{\left(J_{1}-J_{2}\right) w \delta t}{w \delta x} .
$$

And because $\delta x$ is small

$$
J_{2}=J_{1}+\frac{\partial J}{\partial x} \delta x
$$

In the limit where $\delta t \rightarrow 0$, Eqs. (7.9) and (7.10) give

$$
\frac{\partial C}{\partial t}=-\frac{\partial J}{\partial x} \text {. }
$$

By substituting Fick's first law, Eq. (7.6),

$$
\frac{\partial C}{\partial t}=\frac{\partial}{\partial x}\left(D \frac{\partial C}{\partial x}\right),
$$


which simplifies to

$$
\frac{\partial C}{\partial t}=D \frac{\partial^{2} C}{\partial x^{2}}
$$

if $D$ is independent of $x$ and $C$.

\subsubsection{Generalizing Fick's laws to include additional dimen- sions}

So far, the diffusion has been restricted to one dimension on a surface. However, expanding Fick's laws to cover more dimensions is a simple task using the gradient $\nabla$. Thus, Fick's laws can be written as

$$
J=-D \nabla C
$$

and

$$
\frac{\partial C}{\partial t}=D \nabla^{2} C
$$

where $C=C(x, y, z, t)$, and $D$ are tensors. Here, $D$ can vary depending on the direction, although in a cubic lattice it is uniform in all directions [86]. However, on the surface of a cubic structure the direction can be non-uniform for some surface terminations, e.g., $\operatorname{TiN}(110)$.

\subsubsection{Diffusion temperature dependence}

The number of successful jumps per unit time, jump rate, a given atom can perform is dependent on temperature according to

$$
\Gamma=\nu \exp \left(-\frac{\Delta G}{k_{B} T}\right),
$$

where $\nu$ is the attempt frequency of the atoms, and $\Delta G$ is the free energy activation barrier which needs to be overcome in order for an adatom to migrate between jump sites separated by the distance $l[64]$. Eq. (7.5) becomes

$$
D=l^{2} \nu \exp \left(-\frac{\Delta G}{k_{B} T}\right) .
$$

Substituting $\Delta G=\Delta H-T \Delta S$ gives

$$
D=l^{2} \exp \left(\frac{\Delta S}{k_{B}}\right) \exp \left(-\frac{\Delta H}{k_{B} T}\right) .
$$

The diffusivity $D$ can now be divided into one temperature dependent exponential factor and one temperature independent prefactor $D_{0}$, so that

$$
D=D_{0} \exp \left(-\frac{\Delta H}{k_{B} T}\right),
$$




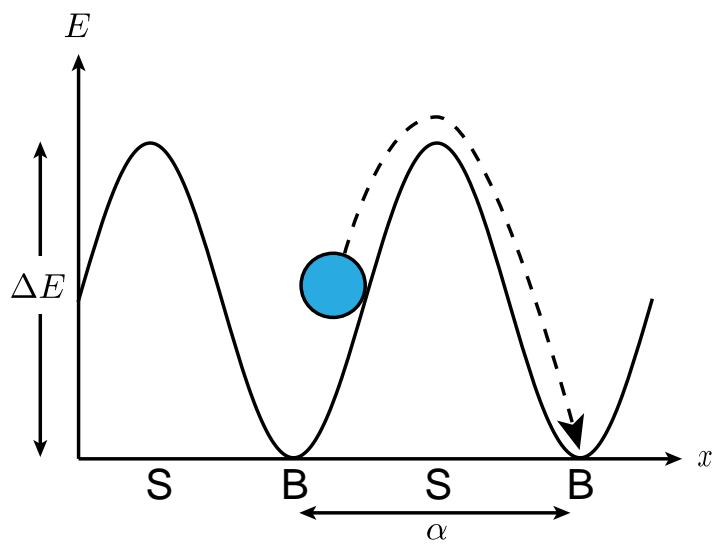

Figure 7.3. The diffusion activation barrier $\Delta E$ is the difference in adsorption energy between a binding site $\mathrm{B}$ and a saddle point $\mathrm{S}$.

and

$$
D_{0}=l^{2} \nu \exp \left(\frac{\Delta S}{k_{B}}\right)=l^{2} \nu_{0},
$$

where $\nu_{0}$ is the frequency prefactor.

Assuming low pressures $\Delta H \approx \Delta E$ [86]. The diffusivity is dependent on the diffusion activation barrier $\Delta E$ according to

$$
D=D_{0} \exp \left(-\frac{\Delta E}{k_{B} T}\right) .
$$

The factor, $D_{0}$ or $\Delta E$, which is more decisive is determined by the temperature. The diffusion activation barrier will be dominating the diffusivity at low temperatures, and at higher temperature $D \rightarrow D_{0}$. Thus, the alloying effects at specific temperatures can differ if the alloying effects mostly the diffusion barrier or the diffusion prefactor.

\subsubsection{Calculating the diffusion activation barrier}

The barrier is determined by the difference in the adatom adsorption energy $E_{a d}$ between a binding site and a saddle point, see Fig. 7.3. The adsorption energy $E_{a d}$ at the coordinate $(x, y)$ is calculated as

$$
E_{a d}(x, y)=E_{\text {config }}(x, y)-\left(E_{\text {slab }}+E_{\text {atom }}^{\text {vacuum }}\right)
$$

the difference between a slab with an adatom and a slab with the adatom in vacuum. The position in the $z$-direction is determined by minimizing the energy.

Calculating the adsorption energy is a straight forward task. A difficulty in obtaining the diffusion activation barrier is finding the binding sites and saddle points. Although this is easy for a simple cubic cell with only one type of atomic 
(a)

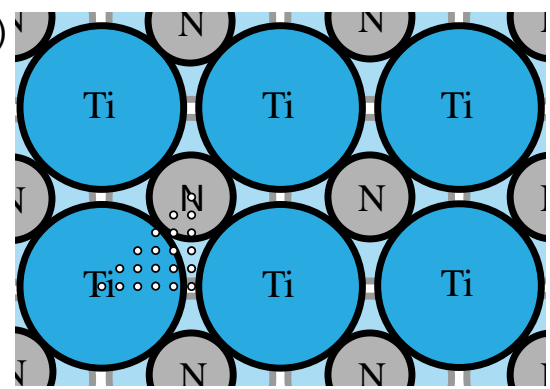

(b)

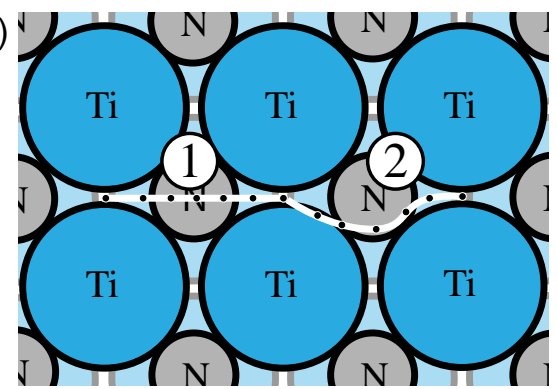

Figure 7.4. (a) Setup for calculating the adsorption energetics by probing the surface in a grid, where the adatom is fixed in-plane position. (b) Illustration of the relaxing effect using the NEB method. The method allows for a linear path 1 to relax into a lowest energy path 2 .

species, the problem becomes many times more complex for alloys with impurities. Two types of methods have been used in this work to find the binding sites and saddle points; by probing the entire surface to find the entire adsorption energy landscape, and using the nudged elastic band method.

\section{The grid probing method}

In the grid method, the adsorption energy landscape is obtained by probing the adsorption energy of an adatom at different positions on the surface by fixing the in-plane relaxation allowing only adatom relaxation perpendicular to the surface, see Fig. 7.4 (a). In addition to the adatom, the topmost layers of the slab are allowed to freely relax.

The accuracy of the adsorption energy landscape is limited by the grid mesh. The grid needs to be fine enough to capture the entire topology of the adsorption energy landscape. This requires many calculations to reach a high accuracy. However, the number of calculations needed can be reduced using the symmetry of the surfaces. Also, since the calculations are independent of each other they are optimally parallelizable.

Calculating the adsorption energy landscape gives an excellent overview of the diffusion landscape, however, it is not as accurate at calculate barrier heights as the nudged elastic band (NEB) method. Furthermore, the complexity of the approach would also increase should we consider more than just a single adatom. For admolecules, other effects, such as orientation, adds extra dimensions to the problem.

\section{The nudged elastic band (NEB) method}

The NEB method [87-89] is designed for finding saddle points and minimum energy paths between known reactants and products. This makes it excellent for finding the lowest energy path between two binding sites. 
The basic setup requires a path between two binding sites, set up with estimated adatom positions through for example linear interpolation, see Fig. 7.4 (b) 1. The atomic positions are then simultaneously relaxed in the $x, y$, and $z$ directions to find the minimum energy path. The relaxations are constrained by a spring force, so that each image finds the lowest energy possible while maintaining equal spacing to neighboring images. Although it is mainly the adatom position that affect the energy in our setup, the surface atoms are also allowed to relax.

This relaxation method used within the NEB method gives a more accurate minimum energy path with fewer calculations than the previous method. However, to effectively use the method, all minima need to be known in order to set up the path. The binding sites depend strongly on the adatom species, so in most cases it is helpful to first get an overview of the energy landscape using the grid approach with a rough grid.

\subsubsection{Approximating the diffusion prefactor}

Estimating the diffusion prefactor $D_{0}$ is difficult both experimentally and theoretically. However, for diffusion of isolated individual adatoms on a surface the fluctuations in the prefactor are generally small and differ little between systems. Still, there are ways to approximate it. Vineyard [90] showed that the prefactor can generally be written as

$$
\nu=\frac{\prod_{j=1}^{n} \nu_{j}}{\prod_{i=1}^{n-1} \nu_{i}},
$$

the ratio of all $n$ vibrations in the system when the adatom is at a binding site and all $n-1$ vibrations when it is at the saddle point. Calculating all of these vibrations is very costly. However, with a simple approximation, that all vibrations other than the adatom vibration in the direction of the jump path are constant, the number of vibrations that are needed are reduced to just one $\nu_{B}$, the vibration frequency in the direction of the jump when the adatom is at a binding site. Although this approximation does not hold for diffusion mechanisms which requires movement of multiple atoms simultaneously, it can serve as a first order description for adatom surface diffusion.

$\nu_{B}$ can be approximated in a classical sense by treating the atom at a binding site as a harmonic oscillator using

$$
\nu_{B}=\frac{1}{2 \pi} \sqrt{\frac{\partial^{2} E / \partial x^{2}}{m}},
$$

where $\partial^{2} E / \partial x^{2}$ is the second derivative of potential energy along the transition path at the binding site and $m$ is the mass of the adatom. Although this is a first order description of $\nu$, the value of going to more intricate descriptions of $\nu$ might be small due to the still present underlying approximations of static lattice-based TST. 


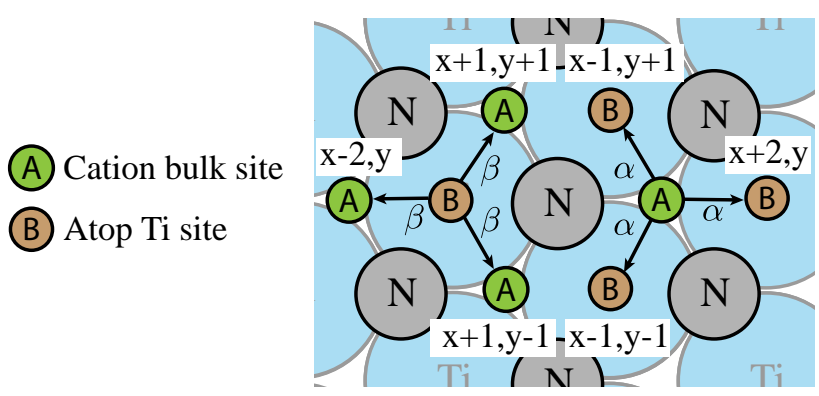

Figure 7.5. Binding site arrangement on a $\operatorname{TiN}(111): \mathrm{N}$ surface where the jump rate from $\mathrm{A}$ to $\mathrm{B}$ is $\alpha$ and the jump rate from $\mathrm{B}$ to $\mathrm{A}$ is $\beta$.

\subsubsection{Diffusivity on surfaces with multiple binding site types}

Calculating the surface diffusivity for systems with only one type of binding site and one atomic species (e.g., a simple cubic (0 01 ) surface) is a relatively simple task, which is probably why it is a common example in the available literature on diffusion. Nevertheless, when considering alloy systems the problem can become quite complex. Some adatom species may have more than one binding site which makes it more difficult to compare the diffusivity of the two species. This added complexity can be seen on the N-terminated TiN(111) surface, Fig. 7.5. Expression for the diffusivity on select complex were derived by Wrigley et al. [91].

Following Wrigley et al. [91], the probability that an adatom is at an A site with the coordinates $(x, y)$ is $p_{x, y}^{A}$, and the probability that it is at a B site with the coordinates $(x, y)$ is $p_{x, y}^{B}$. Because the binding sites are connected as in Fig. 7.5, considering only nearest neighbor jumps with the jump rates are $\Gamma_{\alpha}$ and $\Gamma_{\beta}$ from an $\mathrm{A}$ site respectively $\mathrm{B}$ site, the change with respect to time of the probabilities are described by the Kolmogoroff equations [92]

$$
\frac{d p_{x, y}^{A}}{d t}=-3 \Gamma_{\alpha} p_{x, y}^{A}+\Gamma_{\beta}\left(p_{x+2, y}^{B}+p_{x-1, y+1}^{B}+p_{x-1, y-1}^{B}\right)
$$

and

$$
\frac{d p_{x, y}^{B}}{d t}=-3 \Gamma_{\beta} p_{x, y}^{A}+\Gamma_{\alpha}\left(p_{x-2, y}^{B}+p_{x+1, y+1}^{B}+p_{x+1, y-1}^{B}\right) .
$$

The probability generating functions for the sites are defined as

$$
G^{A}\left(t, z_{1}, z_{2}\right)=\sum_{x} \sum_{y} z_{1}^{x} z_{2}^{y} p_{x, y}^{A}
$$

and

$$
G^{B}\left(t, z_{1}, z_{2}\right)=\sum_{x} \sum_{y} z_{1}^{x} z_{2}^{y} p_{x, y}^{B}
$$

where the sums are taken over the $\mathrm{A}$ and $\mathrm{B}$ sites respectively, and $z_{1}$ and $z_{2}$ are dummy variables in the range $0 \leq z \leq 1$. The generating functions are related to 
the total generating function through

$$
G\left(t, z_{1}, z_{2}\right)=G^{A}\left(t, z_{1}, z_{2}\right)+G^{B}\left(t, z_{1}, z_{2}\right) .
$$

The time derivative of the A site generating function is

$$
\begin{aligned}
\dot{G}^{A}\left(t, z_{1}, z_{2}\right)= & \sum_{x} \sum_{y} z_{1}^{x} z_{2}^{y} \frac{d p_{x, y}^{A}}{d t}= \\
& -\Gamma_{\alpha} \sum_{x} \sum_{y} p_{x, y}^{A}+\Gamma_{\beta}\left(\sum_{x} \sum_{y} z_{1}^{x} z_{2}^{y} p_{x+2, y}^{B}\right. \\
& \left.+\sum_{x} \sum_{y} z_{1}^{x} z_{2}^{y} p_{x-1, y+1}^{B}+\sum_{x} \sum_{y} z_{1}^{x} z_{2}^{y} p_{x-1, y-1}^{B}\right),
\end{aligned}
$$

which can be more conveniently written as

$$
\begin{aligned}
\dot{G}^{A}\left(t, z_{1}, z_{2}\right)= & -3 \Gamma_{\alpha} \sum_{x} \sum_{y} p_{x, y}^{A} \\
+\Gamma_{\beta}( & \frac{1}{z_{1}^{2}} \sum_{x} \sum_{y} z_{1}^{x+2} z_{2}^{y} p_{x+2, y}^{B} \\
& +\frac{z_{1}}{z_{2}} \sum_{x} \sum_{y} z_{1}^{x-1} z_{2}^{y+1} p_{x-1, y+1}^{B} \\
& \left.+z_{1} z_{2} \sum_{x} \sum_{y} z_{1}^{x-1} z_{2}^{y-1} p_{x-1, y-1}^{B}\right) .
\end{aligned}
$$

A shorter way to represent the equation is

$$
\begin{aligned}
\dot{G}^{A}\left(t, z_{1}, z_{2}\right)= & -3 \Gamma_{\alpha} G^{A}\left(t, z_{1}, z_{2}\right) \\
& +\Gamma_{\beta}\left(\frac{1}{z_{2}^{2}}+\frac{z_{1}}{z_{2}}+z_{1} z_{2}\right) G^{B}\left(t, z_{1}, z_{2}\right),
\end{aligned}
$$

and in the same way

$$
\begin{aligned}
\dot{G}^{B}\left(t, z_{1}, z_{2}\right)= & -3 \Gamma_{\beta} G^{B}\left(t, z_{1}, z_{2}\right) \\
& +\Gamma_{\alpha}\left(z_{1}^{2}+\frac{1}{z_{1} z_{2}}+\frac{z_{2}}{z_{1}}\right) G^{A}\left(t, z_{1}, z_{2}\right) .
\end{aligned}
$$

Using

$$
u=\frac{1}{3}\left(\frac{1}{z_{2}^{2}}+\frac{z_{1}}{z_{2}}+z_{1} z_{2}\right)
$$

and

$$
v=\frac{1}{3}\left(z_{1}^{2}+\frac{1}{z_{1} z_{2}}+\frac{z_{2}}{z_{1}}\right)
$$


Eq. (7.32) and (7.33) can be written as

$$
\dot{G}^{A}\left(t, z_{1}, z_{2}\right)=-3 \Gamma_{\alpha} G^{A}\left(t, z_{1}, z_{2}\right)+3 \Gamma_{\beta} u G^{B}\left(t, z_{1}, z_{2}\right)
$$

and

$$
\dot{G}^{B}\left(t, z_{1}, z_{2}\right)=-3 \Gamma_{\beta} G^{B}\left(t, z_{1}, z_{2}\right)+3 \Gamma_{\alpha} v G^{A}\left(t, z_{1}, z_{2}\right) .
$$

If the adatom starts at the $(x, y)=(0,0)$ position then $G^{A}\left(0, z_{1}, z_{2}\right)=z_{1} z_{2}$ and $G^{B}\left(0, z_{1}, z_{2}\right)=0 .^{2}$ Thus, the Laplace transform of the left hand sides of Eq. (7.36) and (7.37) are

$$
\begin{aligned}
\mathcal{L}\left(\dot{G}^{A}\left(t, z_{1}, z_{2}\right)\right)= & s \mathcal{L}\left(G^{A}\left(t, z_{1}, z_{2}\right)\right)+G^{A}\left(0, z_{1}, z_{2}\right)= \\
& s \mathcal{L}\left(G^{A}\left(t, z_{1}, z_{2}\right)\right)+z_{1} z_{2}
\end{aligned}
$$

and

$$
\begin{aligned}
\mathcal{L}\left(\dot{G}^{B}\left(t, z_{1}, z_{2}\right)\right)= & s \mathcal{L}\left(G^{B}\left(t, z_{1}, z_{2}\right)\right)+G^{B}\left(0, z_{1}, z_{2}\right)= \\
& s \mathcal{L}\left(G^{B}\left(t, z_{1}, z_{2}\right)\right),
\end{aligned}
$$

and the right hand sides are

$$
-3 \Gamma_{\alpha} \mathcal{L}\left(G^{A}\left(t, z_{1}, z_{2}\right)\right)+3 \Gamma_{\beta} u \mathcal{L}\left(G^{B}\left(t, z_{1}, z_{2}\right)\right)
$$

and

$$
-3 \Gamma_{\beta} \mathcal{L}\left(G^{B}\left(t, z_{1}, z_{2}\right)\right)+3 \Gamma_{\alpha} v \mathcal{L} .\left(G^{A}\left(t, z_{1}, z_{2}\right)\right) .
$$

Solving the equation system gives

$$
\begin{aligned}
& \mathcal{L}\left(G^{A}\left(t, z_{1}, z_{2}\right)\right)=\frac{\left(s+3 \Gamma_{\beta}\right) z_{1} z_{2}}{(s+q)(s+r)}, \\
& \mathcal{L}\left(G^{B}\left(t, z_{1}, z_{2}\right)\right)=\frac{3 v \Gamma_{\alpha} z_{1} z_{2}}{(s+q)(s+r)},
\end{aligned}
$$

where

$$
\begin{aligned}
& q \equiv \frac{3\left(\Gamma_{\alpha}+\Gamma_{\beta}\right)}{2}\left(1+\sqrt{1-\frac{4 \Gamma_{\alpha} \Gamma_{\beta}(1-u v)}{\left(\Gamma_{\alpha}+\Gamma_{\beta}\right)^{2}}}\right), \\
& r \equiv \frac{3\left(\Gamma_{\alpha}+\Gamma_{\beta}\right)}{2}\left(1-\sqrt{1-\frac{4 \Gamma_{\alpha} \Gamma_{\beta}(1-u v)}{\left(\Gamma_{\alpha}+\Gamma_{\beta}\right)^{2}}}\right) .
\end{aligned}
$$

\footnotetext{
${ }^{2}$ It is also possible to choose the B-site as the starting position. Then $G^{A}\left(0, z_{1}, z_{2}\right)=0$ and $G^{B}\left(0, z_{1}, z_{2}\right)=z_{1}^{2}$.
} 
The inverse Laplace transforms of Eq. (7.42) and (7.43) will then give the generating functions

$$
G^{A}\left(t, z_{1}, z_{2}\right)=\frac{z_{1} z_{2}}{q-r}\left(\left(q-3 \Gamma_{\beta}\right) e^{-q t}-\left(r-3 \Gamma_{\beta}\right) e^{-r t}\right)
$$

and

$$
G^{B}\left(t, z_{1}, z_{2}\right)=\frac{3 v \Gamma_{\alpha} z_{1} z_{2}}{q-r}\left(e^{-r t}-e^{-q t}\right) .
$$

The probability $P^{A}$ that an adatom is at an A site is simply $G^{A}(t, 1,1)$, since

$$
\begin{array}{r}
P^{A}=\sum_{x} \sum_{y} p_{x, y}^{A}=G^{A}(t, 1,1) \\
=\frac{\Gamma_{\alpha} e^{-3\left(\Gamma_{\alpha}+\Gamma_{\beta}\right) t}+\Gamma_{\beta}}{\Gamma_{\alpha}+\Gamma_{\beta}} .
\end{array}
$$

The probability $P^{B}$ is likewise

$$
\begin{array}{r}
P^{B}=\sum_{x} \sum_{y} p_{x, y}^{B}=G^{B}(t, 1,1) \\
=\frac{\Gamma_{\alpha} e^{-3\left(\Gamma_{\alpha}+\Gamma_{\beta}\right) t}-\Gamma_{\alpha}}{\Gamma_{\alpha}+\Gamma_{\beta}} .
\end{array}
$$

From the generating function it is now possible to access the moments in the $x$-direction $\left\langle x^{n}\right\rangle$ through the relation

$$
\left\langle x^{n}\right\rangle=\sum_{x} x^{n} p_{x}=\left[\left(z_{1} \frac{\partial}{\partial z_{1}}\right)^{n} G\left(t, z_{1}, 1\right)\right]_{z_{1}=1}
$$

where moments are in the units $a / \sqrt{6}$, where $a$ is the lattice constant ${ }^{3}$. The average displacement $\langle x\rangle$ is

$$
\langle x\rangle=1
$$

and the mean-square value $\left\langle x^{2}\right\rangle$ is

$$
\left\langle x^{2}\right\rangle=\frac{2 \Gamma_{\alpha}\left(\Gamma_{\beta}-\Gamma_{\alpha}\right) e^{-3\left(\Gamma_{\alpha}+\Gamma_{\beta}\right) t}+\Gamma_{\beta}^{2}+12 \Gamma_{\alpha} \Gamma_{\beta}^{2} t+3 \Gamma_{\alpha}^{2}\left(1+4 \Gamma_{\beta} t\right)}{\left(\Gamma_{\alpha}+\Gamma_{\beta}\right)^{2}} .
$$

The fluctuation $\left\langle\Delta x^{2}\right\rangle$ in $x$ is

$$
\left\langle\Delta x^{2}\right\rangle=\left\langle x^{2}\right\rangle-\langle x\rangle^{2}=\frac{2 \Gamma_{\alpha}\left(\Gamma_{\alpha}-\Gamma_{\beta}\right)\left(1-e^{-3\left(\Gamma_{\alpha}+\Gamma_{\beta}\right) t}\right)}{\left(\Gamma_{\alpha}+\Gamma_{\beta}\right)^{2}}+\frac{12 \Gamma_{\alpha} \Gamma_{\beta}}{\Gamma_{\alpha}+\Gamma_{\beta}} t .
$$

The first term in Eq. (7.53) accounts for the transient behavior at the beginning of diffusion where the starting site is important. For long diffusion times, the equation can be reduced to

$$
\left\langle\Delta x^{2}\right\rangle=12 \frac{\Gamma_{\alpha} \Gamma_{\beta}}{\Gamma_{\alpha}+\Gamma_{\beta}} t
$$

\footnotetext{
${ }^{3}$ Moments in the $y$-direction will in this symmetry be in the unit $a /(2 \sqrt{2})$
} 
where $x$ is still in units of $a / \sqrt{6}$, converted to $m^{2} / s$ the equation becomes

$$
\left\langle\Delta a^{2}\right\rangle=2 a^{2} \frac{\Gamma_{\alpha} \Gamma_{\beta}}{\Gamma_{\alpha}+\Gamma_{\beta}} t
$$

The diffusivity $\mathrm{D}$ is derived from the fluctuation $\left\langle\Delta a^{2}\right\rangle$ through the Einstein relation

$$
\left\langle\Delta a^{2}\right\rangle=2 D t
$$

which means that the surface diffusivity is

$$
D=a^{2} \frac{\Gamma_{\alpha} \Gamma_{\beta}}{\Gamma_{\alpha}+\Gamma_{\beta}} .
$$

Note that $D$ at this point is in the SI units $\mathrm{m}^{2} / \mathrm{s}$, but the commonly used unit is $\mathrm{cm}^{2} / \mathrm{s}$.

\section{$\operatorname{TiN}\left(\begin{array}{lll}0 & 0 & 1\end{array}\right)$}

This method can also be applied to calculate the diffusivity of Ti adatoms on the TiN(l $\left.\begin{array}{lll}0 & 1\end{array}\right)$ surface. The unit cell for this surface is cubic with three types of sites: one bulk site with four connecting fourfold hollow sites (two outside the unit cell), one fourfold hollow site with connecting bulk sites in the $\langle 110\rangle$ direction, and one fourfold hollow site with connecting bulk sites in the $\langle\overline{1} 10\rangle$ direction. The resulting expression for this site is

$$
D_{\mathrm{Ti}}^{\mathrm{TiN}(0001)}=\left(\frac{a}{2}\right)^{2} \frac{2 \Gamma_{\alpha} \Gamma_{\beta}}{2 \Gamma_{\alpha}+\Gamma_{\beta}},
$$

where $\Gamma_{\alpha}$ is the jump rate from the bulk site, $\Gamma_{\beta}$ is the jump rate from the hollow sites, and $a$ is the distance between bulk sites.

This method can also be applied to more complex surfaces, such as supercells. Although the equations become increasingly complicated and many more barriers need to be calculated, they do not require extensive statistical probing as, e.g., KMC. Using this method could, therefore, be much less computationally demanding.

\subsection{Hard coatings}

Hard coatings, typically in the form of a few $\mu \mathrm{m}$ thick films, have been used for many decades to increase the lifetime of tools, such as cutting tools, drill heads etc. $[93,94]$. One of the most successful hard coatings is cubic rocksalt-TiN, which can be recognized by its characteristic gold color.

Alloying TiN with AlN, has been found to improve both film hardness [8, 10, 95-98] and oxidation resistance [9, 99-101]. This makes it suitable for a wide range of applications, e.g. high-speed cutting tools [93] and bio-implant coatings [94].

$\mathrm{Ti}_{1-x} \mathrm{Al}_{x} \mathrm{~N}$ is a metastable alloy which can be synthesized using $\mathrm{PVD}$, allowing the growth of the film to occur at low temperatures so that there is no bulk 


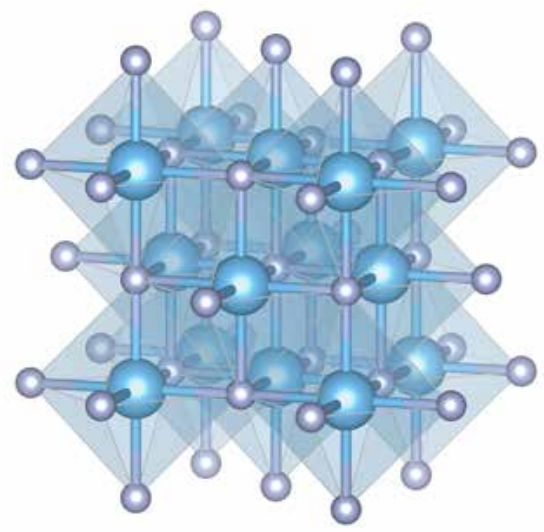

Figure 7.6. The cubic octahedraly coordinated rocksalt crystal structure of TiN.

diffusion, and only limited surface diffusion. However, when it is subjected to high temperatures, such as during cutting operations, bulk diffusion is activated and the film starts to decompose into rocksalt-TiN and either rocksalt-AlN or wurtziteAlN [102-107]. This is part of the reason behind the improved wear resistance observed experimentally $[102,103,105,106]$ and explained theoretically $[102,106$, 108-110].

The atomic level kinetics during $\mathrm{Ti}_{1-x} \mathrm{Al}_{x} \mathrm{~N}$ growth is pivotal to understanding the micro- and nano-structural evolution of the film. Nevertheless, it is difficult to investigate this experimentally, due to the very short time scales involved in surface diffusion events. Instead, it is possible to use TST [90, 111]. This approach has been used to gain valuable knowledge about the surface kinetics of elemental metals $[15,112,113]$, TiC $[114,115]$ and the parent compounds TiN $[16,115-$ 118 ] and $\mathrm{AlN}$ [119]. Modeling the surface kinetics for an alloy like $\mathrm{Ti}_{1-x} \mathrm{Al}_{x} \mathrm{~N}$, however, is more complex because of configurational disorder effects. Two suitable starting points is to either study the effects which the added $\mathrm{Al}$ has on the TiN surface energetics by investigating the fully mixed $\mathrm{Ti}_{0.5} \mathrm{Al}_{0.5} \mathrm{~N}$, or investigate the much more dilute case where the local and chemical effects are distinguishable from those originating from explicit configurational disorder.

\subsection{Disordered $\mathrm{Ti}_{1-x} \mathrm{Al}_{x} \mathrm{~N}$ surface diffusion}

Introducing additional atomic species in a material will, with varying importance, have effects on the diffusion. By understanding how diffusion on an alloy surface is different from a pure surface it is possible to predict or explain differences in morphology, preferred grain orientations, etc., between alloy and pure compound growth. It is possible to get a very accurate picture of the surface energetics that influences the adatom migrations using DFT calculations and the methods described in the previous sections in this chapter. We have, therefore, focused 
our study on $\mathrm{TiN}$ and $\mathrm{Ti}_{1-x} \mathrm{Al}_{x} \mathrm{~N}$, which are experimentally and theoretically well investigated and, thus, exemplary model systems.

\subsubsection{Pure TiN}

There are three commonly occurring surface orientations in the grains created during TiN growth: (001), (110) and (111). Of these, the (001) and (111) grains are the most common, since they have lower surface energy than the $\left(\begin{array}{ll}1 & 10\end{array}\right)$ surface. The surface adatom energetics of a Ti adatom on the three surfaces is shown in Fig. 7.7, where the potential energy is relative to the strongest binding

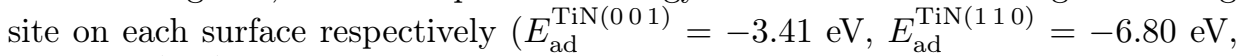

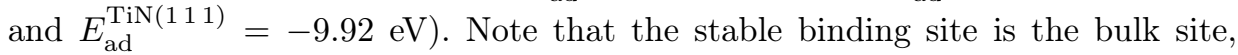
except on the $\operatorname{TiN}(001)$ surface, where the fourfold hollow site is stable and the bulk site is only a metastable.

The large differences between the surfaces orientations are apparent in Fig. 7.7; TiN(0 01$)$ has the lowest diffusion activation energy barriers, TiN(111) has the second lowest, and TiN(110) has the highest. Thus, the adatom mobility is highest on $\operatorname{TiN}(001)$, slower on $\operatorname{TiN}(111)$, and slowest on $\operatorname{TiN}(110)$. Furthermore, the large difference between the energy barriers for crossing Ti surface atoms compared to $\mathrm{N}$ surface atoms on TiN(110) strongly indicates one dimensional diffusion on this surface.

The surface energetics of $\mathrm{Al}$ adatoms are very similar to that of $\mathrm{Ti}$ adatoms, with slightly different binding energies and diffusion barriers. However, there is one important difference, $\mathrm{Al}$ adatoms on $\mathrm{TiN}(001)$ only bind to the bulk site. Although the diffusion barrier for $\mathrm{Al}$ adatoms on $\mathrm{TiN}(001)$ is $17 \%$ higher than the Ti adatom diffusion barrier making each migration attempt less likely to succeed, each successful step takes the $\mathrm{Al}$ adatom twice as far. As a result, at high enough temperatures, $\mathrm{Al}$ adatoms will start to migrate faster than Ti adatoms.

$\mathrm{N}$ adatom surface energetics are distinctly different from the metal adatom energetics since $\mathrm{N}$ is smaller and can access narrower adsorption sites such as the threefold site on $\mathrm{TiN}\left(\begin{array}{lll}0 & 0 & 1\end{array}\right)$. The binding energy of $\mathrm{N}$ is very weak in comparison to free $\mathrm{N}_{2}$, in fact it is positive at most binding sites. However, compared to a single free $\mathrm{N}$ atom in vacuum the resulting adsorption energy is $5.19 \mathrm{eV}$ stronger. Thus, $\mathrm{N}$ is very likely to desorb when it finds another free $\mathrm{N}$ adatom. Furthermore, on the $\operatorname{TiN}\left(\begin{array}{lll}0 & 0 & 1\end{array}\right)$ surface it prefers the threefold hollow site near a $\mathrm{N}$ atom in the surface layer where it can desorb as $\mathrm{N}_{2}$ if the temperature is high enough [120].

\subsubsection{Disordered $\mathrm{Ti}_{0.5} \mathrm{Al}_{0.5} \mathrm{~N}$}

To study surface diffusion on disordered $\mathrm{Ti}_{0.5} \mathrm{Al}_{0.5} \mathrm{~N}$ surfaces is a much more difficult task than the ordered TiN surfaces. In Paper VII we approached the problem by using the SQS method to generate a structure model with the same 50/50 composition in each layer.

The adsorption energy landscape of the $\mathrm{TiN}(001)$ and $\mathrm{Ti}_{0.5} \mathrm{Al}_{0.5} \mathrm{~N}(001)$ are shown in Fig. 7.8. By comparing the two surfaces it becomes apparent that the disordered surface is much more complex, with many unique local features. For 


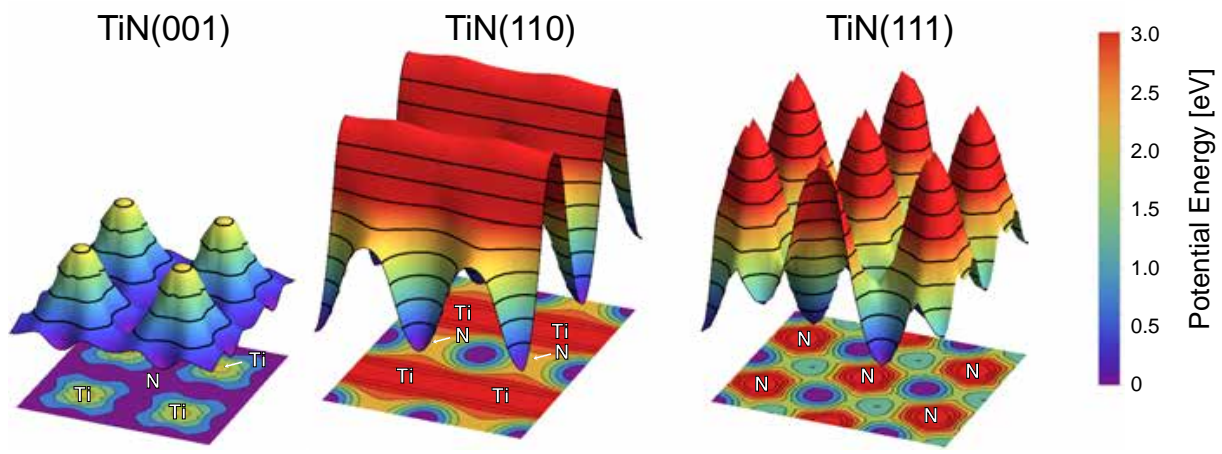

Figure 7.7. The potential energy relative to the respective strongest binding site for a $\mathrm{Ti}$ adatom on the $\operatorname{TiN}(001)$, $\operatorname{TiN}(110)$, and $\operatorname{TiN}(111)$ surfaces. Contour lines correspond to a $0.5 \mathrm{eV}$ energy difference in adsorption energies. Adopted from Paper VIII.

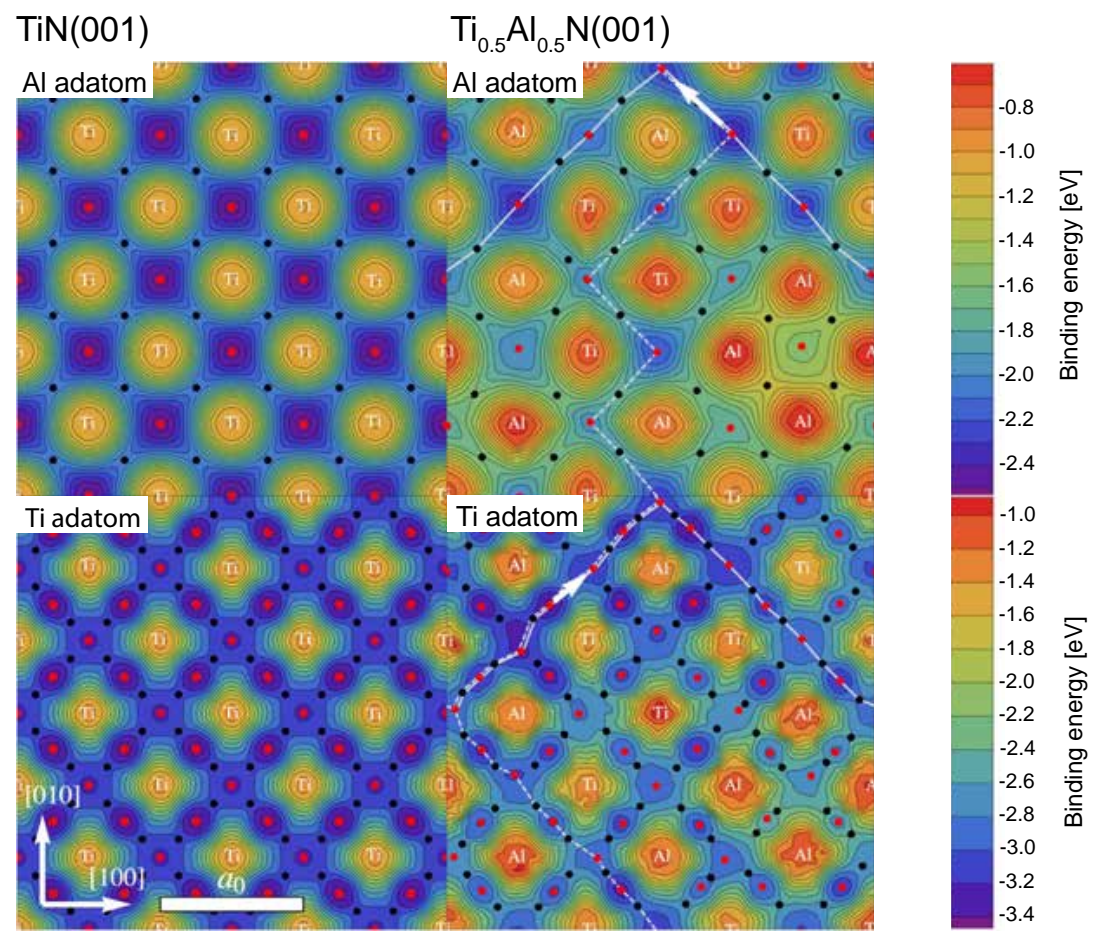

Figure 7.8. The binding energy of $\mathrm{Al}$ and $\mathrm{Ti}$ adatoms on $\mathrm{TiN}(001)$ and $\mathrm{Ti}_{0.5} \mathrm{Al}_{0.5} \mathrm{~N}(001)$. Red dots mark local minimas, black dots mark barrier saddle points, and white solid and dotted lines mark preferential diffusion paths. Adopted from Paper VII. 
example, the $\mathrm{Al}$ rich environments are energetically unfavorable to bind on top of for both $\mathrm{Ti}$ and $\mathrm{Al}$ adatoms. The key difference between $\mathrm{Al}$ and $\mathrm{Ti}$ adatom energy surface is that the configurational disorder only increases the diffusion barriers for $\mathrm{Ti}$ adatoms while the $\mathrm{Al}$ adatom diffusion barriers along the high diffusion paths are largely unaffected, see Paper VII.

In order to study how the configurational disorder affects adatom mobility in the two cases we generated a large circular slab consisting of connected SQS surfaces. The surfaces were connected in two different ways, all SQS surfaces oriented in the same direction, alternatively in random directions. By applying transition state theory and a probabilistic approach we analyze the mobility of $\mathrm{Ti}$ and $\mathrm{Al}$ adatoms on the two surfaces. This simulation confirmed that the increased barrier for $\mathrm{Ti}$ adatoms together with the asymmetric adsorption energy surfaces dramatically decreases the Ti adatom mobility. Nonetheless, a limitation with this SQS approach is that it is difficult to distinguish chemistry effects from configurational effects.

\subsubsection{Effect of single Al substitution}

In Paper VIII we separate the chemical effects from the configurational by investigating the effects on $\mathrm{Ti}, \mathrm{Al}$, and $\mathrm{N}$ adatom diffusion in the dilute $\mathrm{Al}$ case, where one out of nine Ti surface atoms are substituted with an $\mathrm{Al}$ atom in the $\mathrm{TiN}\left(\begin{array}{ll}0 & 0\end{array}\right)$, TiN(1 10$)$, and TiN(1 11 ) surfaces. We then investigated these models using the grid probing technique and NEB method described in Sec. 7.1.5.

\section{$\operatorname{TiN}\left(\begin{array}{lll}0 & 0 & 1\end{array}\right)$}

The changes in adsorption energies on the $\operatorname{TiN}\left(\begin{array}{lll}0 & 0 & 1\end{array}\right)$ surface due to the substitution are shown in Fig.7.9. Here, it is apparent that $\mathrm{Ti}$ are energetically most affected by the $\mathrm{Al}$ substitution, while the $\mathrm{Al}$ adatom pathways are barely affected by the substitution.

Ti adatoms bind $0.2 \mathrm{eV}$ stronger in the fourfold hollow site than on the pure TiN(0 01$)$ surface. Moreover, the barrier from the fourfold site to the closest bulk site is the equal in size to that of the pure surface. However, the reverse transition, from the closest bulk site back to the fourfold site, is only hindered by a minimal energy barrier. This means that a Ti adatom at the bulk site closest to an $\mathrm{Al}_{\mathrm{Ti}}$ atom is more likely to fall in to the fourfold site than to move away from the $\mathrm{Al}_{\mathrm{Ti}}$ atom. The Ti adatom is, therefore, required to move from the $\mathrm{Al}_{\mathrm{Ti}}$ adjacent fourfold site to the next fourfold site in one go, effectively separated by an energy barrier approximately twice that of a transition from a normal fourfold site on pure TiN. Thus, the fourfold site is acting as Ti adatoms trapping site, slowing down $\mathrm{Ti}$ adatom migration in line with what was observed on the disordered $\mathrm{Ti}_{0.5} \mathrm{Al}_{0.5} \mathrm{~N}$ surface.

The stronger $\mathrm{Ti}$ binding energy near the $\mathrm{Al}_{\mathrm{Ti}}$ atom is caused by the broken $\mathrm{Ti}-\mathrm{Ti}$ bond in the surface layer. This frees up an extra valence electron that can be used for binding between the remaining $\mathrm{Ti}$ atoms and a Ti adatom. The extra bonding is, however, only available at the fourfold hollow site, which is only 
(a) Ti adatom

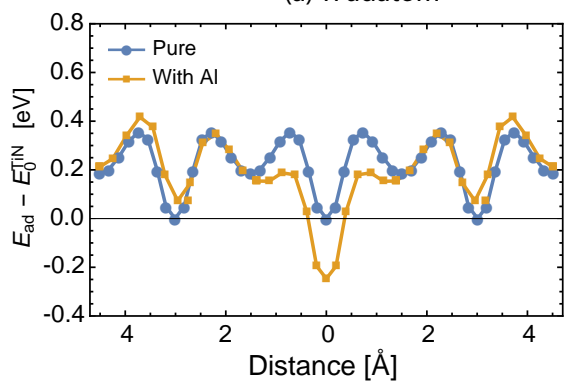

(b) Al adatom

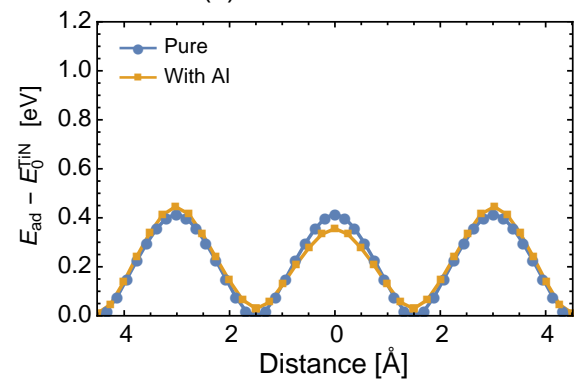

Figure 7.9. Ti, and $\mathrm{Al}$ diffusion pathways on $\operatorname{TiN}\left(\begin{array}{lll}0 & 0 & 1\end{array}\right)$ and $\operatorname{TiN}\left(\begin{array}{lll}0 & 0 & 1\end{array}\right)$ with one $\mathrm{Al}_{\mathrm{Ti}}$ substitution. Distance is measured from the part of the diffusion path closest to the $\mathrm{Al}_{\mathrm{Ti}}$ atom. Adopted from Paper VIII.

(a) Ti adatom

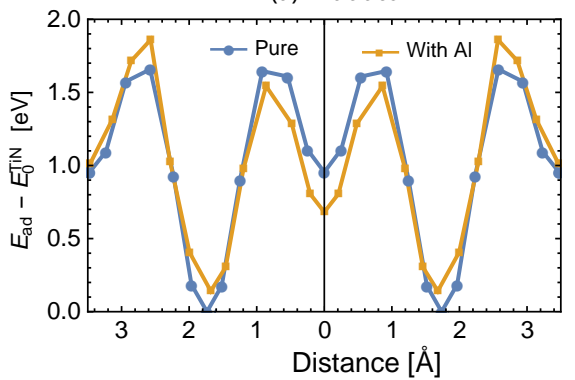

(b) Al adatom

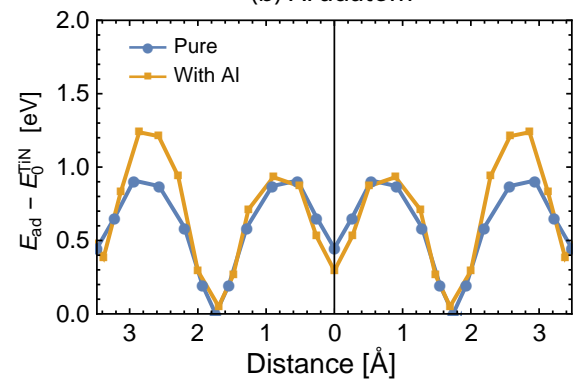

Figure 7.10. $\mathrm{Ti}$, and $\mathrm{Al}$ diffusion pathways on $\mathrm{TiN}(111)$ and $\mathrm{TiN}(111)$ with one $\mathrm{Al}_{\mathrm{Ti}}$ substitution. Distance is measured from the part of the diffusion path closest to the $\mathrm{Al}_{\mathrm{Ti}}$ atom. Adopted from Paper VIII.

(a) Ti adatom

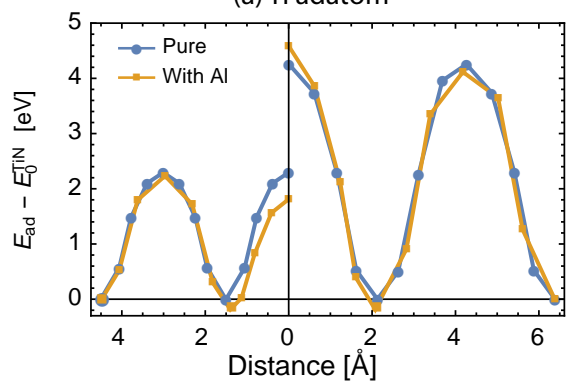

(b) Al adatom

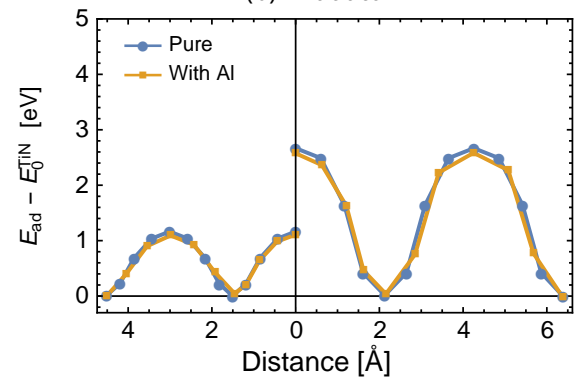

Figure 7.11. $\mathrm{Ti}$, and $\mathrm{Al}$ diffusion pathways on $\mathrm{TiN}(110)$ and $\mathrm{TiN}(110)$ with one $\mathrm{Al}_{\mathrm{Ti}}$ substitution. Distance is measured from the part of the diffusion path closest to the $\mathrm{Al}_{\mathrm{Ti}}$ atom. Adopted from Paper VIII. 
accessible by $\mathrm{Ti}$ and not $\mathrm{Al}$ adatoms. This can be attributed to that $\mathrm{Ti}$ atoms have $d$-electrons while $\mathrm{Al}$ atoms only have $s$ - and $p$-electrons.

\section{$\operatorname{TiN}(111)$}

The $\mathrm{Ti}$ and $\mathrm{Al}$ adatom diffusion pathways on $\mathrm{TiN}(111)$ are presented in Fig. 7.10. $\mathrm{Ti}$ adatoms are the slowest diffusing species also after the $\mathrm{Al}_{\mathrm{Ti}}$ substitution, with only a moderate decrease in mobility due to the stronger binding at the atop $\mathrm{Al}_{\mathrm{Ti}}$ position. For $\mathrm{Al}$ adatoms the substitution has greater effect, because an $\mathrm{Al}$ adatom trap is created at the atop $\mathrm{Al}_{\mathrm{Ti}}$ site and the neighboring bulk sites.

\section{$\operatorname{TiN}(110)$}

The effect of $\mathrm{Al}_{\mathrm{Ti}}$ substitution on $\mathrm{TiN}(110)$, Fig. 7.11, is less dramatic than on the $\operatorname{TiN}(001)$ and $\mathrm{TiN}(111)$ surfaces. The $\mathrm{Al}$ adatom diffusion pathways are barely influenced by the substitution. The changes to the Ti adatom diffusion pathway are too small compared to the activation barrier for them to cause any distinguishable effects to Ti adatom mobility.

\section{$\mathrm{N}$ adatom mobility}

The general effect of $\mathrm{Al}_{\mathrm{Ti}}$ substitution on the $\mathrm{N}$ adatom diffusion pathways is a decrease in bonding strength closest to the $\mathrm{Al}_{\mathrm{Ti}}$ atom. There is, however, no dramatic effect that would influence the relative $\mathrm{N}$ adatom mobility on the different surfaces in the same way as is observed for $\mathrm{Ti}$ and $\mathrm{Al}$ adatoms.

\section{Implication for TiN and TiAlN film growth}

Our calculations of the surface energies for the three surface orientations agree well with experimental results showing that TiN films grown on amorphous substrates the film will be dominated by an approximately equal number of (0 01 ) and (111) oriented islands [121, 122]. (110) oriented islands are, however, very rare. The competition between the $\left(\begin{array}{llll}0 & 0 & 1\end{array}\right)$ and (1 111$)$ surface orientations is, therefore, most important.

The mobility of $\mathrm{Ti}$ adatoms is faster on the $\operatorname{TiN}(001)$ surface than on the TiN(1 1 1) surface. They will therefore be more likely to move off ( 001$)$ islands and to stick to (111) islands. This will cause (l0 01 ) islands to grow laterally while (111) islands grow more three-dimensionally. However, when the islands start to coalesce the effect will be reversed. Adatoms deposited on top of a (001) island will likely diffuse to the island edge and stick to the (111) island. The (111) island will, eventually, start to overgrow the (001) islands. Thus, (1 111$)$ islands will after continued growth be the dominating orientation, inline with what is observed experimentally [121, 122] and theoretically by Gall et al. [16].

$\mathrm{Al}$ adatoms also move faster on the (001) surface than on the (111) surface. However, the kinetic advantage is much less for $\mathrm{Al}$ adatoms than for Ti adatoms. $\mathrm{Al}$ adatoms will, therefore, not contribute to the (111) development as much as $\mathrm{Ti}$ adatoms. Furthermore, when $\mathrm{Al}$ is incorporated in the islands, Ti adatom mobility 
(a) Ti diffusion path

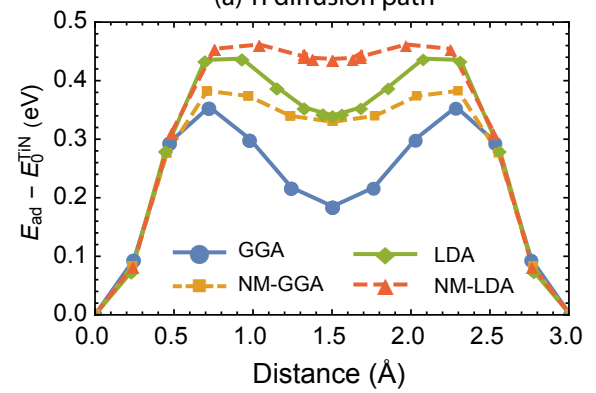

(b) $\operatorname{TiN}(001)$ Diffusivity

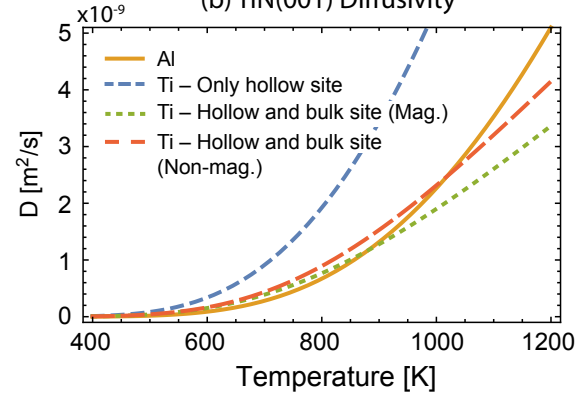

Figure 7.12. (a) Effect of spin polarization on magnetic adatoms. GGA and LDA $\mathrm{Ti}$ adatom $\mathrm{TiN}(001)$ diffusion path with and without spin polarization. Distance is measured from a stable fourfold hollow site. Adopted from Paper VIII. (b) Comparison of $\mathrm{Ti}$ and $\mathrm{Al}$ adatom diffusivity on $\mathrm{TiN}(001)$ as a function of temperature with respect to different approximations for the Ti adatom energy landscape.

is reduced on the (001) surface due to the created Ti trapping sites. This in turn results in decreased (111) development. Extrapolating from our results, we expect that $\operatorname{TiAlN}\left(\begin{array}{lll}0 & 1\end{array}\right)$ will to some extent have higher TiN content and TiAlN(111) will have higher AlN content.

Our results provides a possible explanation for the observed shift from (111) preferred growth to (0 01 ) preferred growth when $\mathrm{Al}$ is added to TiN [98, 123], as well the higher AlN content in the (111) grains [123]. However, not every aspect can be explained by the mechanisms studied in our work. Additional studies are needed to fully understand the growth process, e.g., additional $\mathrm{Al}_{\mathrm{Ti}}$ substitutions, step edge mass transports, admolecule migrations, etc.

\subsubsection{Spin polarization effects on adatom diffusion}

The investigation in Paper VIII also revealed that it is important to include spin polarization effects to accurately model the diffusion path of certain adatom species. This is the case even though pure TiN is a material without intrinsic spin, which is likely the case why it was not considered in previous studies by, e.g., Gall et al. [16]. We found that $\mathrm{Ti}$ adatom spin polarization has substantial effect on local adsorption energies. Although the adsorption energy at the fourfold hollow site on the $\mathrm{TiN}\left(\begin{array}{ll}0 & 0\end{array}\right)$ in unchanged, there is a dramatic effect at the bulk site. The binding energy is approximately four times stronger at the bulk site when spin is considered, effectively increasing the barrier from the bulk site to the fourfold hollow site. Qualitatively the barrier is increased to almost half of the barrier in the opposite direction, see Fig. 7.12 (a). We also confirm that the effect is present for both LDA and GGA, although the barriers are slightly higher with LDA.

The implications for adatom mobility can be dramatic depending on if spin is treated. The diffusivity of $\mathrm{Ti}$ adatoms using different approaches is compared to the diffusivity of $\mathrm{Al}$ adatoms in Fig. 7.12 (b). It is clear that if we only consider that $\mathrm{Ti}$ adatoms would migrate from one fourfold site directly to another, over a barrier 
the size of the Ti diffusion activation barrier we would see a very fast diffusion, and $\mathrm{Ti}$ will always have a higher mobility than $\mathrm{Al}$ adatoms. The two curves, where both the hollow and bulk sites are taken into account according to Eq. (7.58), will both reach a critical temperature after which $\mathrm{Al}$ adatoms will diffuse faster than $\mathrm{Ti}$ adatoms. Accounting for spin polarisation will, nonetheless, shift the crossing point by approximately $150 \mathrm{~K}$ (from $870 \mathrm{~K}$ to $1020 \mathrm{~K}$ ). The difference in diffusivity is increasing with temperature, at $1020 \mathrm{~K}$ it is approximately $22 \%$. At some point, however, additional migration mechanisms will become available that could be more important than the effect of spin polarization.

It is possible that the effect of spin polarization is even more prominent for other adatom species on TiN, e.g., Cr or Fe, or on other material surfaces. Thus, the effect is worth investigating further. 


\section{CHAPTER 8}

My research on multicomponent nitrides has been focused on piezoelectric, thermodynamic, and kinetic properties with mainly thin film applications in mind. This chapter contains conclusions I draw from my contributions to these fields.

\subsection{Piezoelectric nitrides}

In my investigation of the piezoelectric properties of ternary alloys of group IIIA and group IIIB nitrides I found that the large increase in the piezoelectric response, with respect to that of the pure IIIA nitride, first observed for $\mathrm{Sc}_{x} \mathrm{Al}_{1-x} \mathrm{~N}$ alloys $[11,58]$ is in fact a general feature for all of these alloys. The quantitative extent of the increase differs, however, between the alloys. For example, in $\mathrm{Y}_{0.5} \operatorname{In}_{0.5} \mathrm{~N}$, the clamped piezoelectric response $d_{33, f}$ is increased from $4.60 \mathrm{pC} / \mathrm{N}$ to $23.31 \mathrm{pC} / \mathrm{N}$.

In addition to the ternary alloys, I have also shown that large piezoelectric response can be introduced in quarternary alloys, where AlN is alloyed with a ternary $\mathrm{TM}_{0.5} \mathrm{M}_{0.5} \mathrm{~N}(\mathrm{TM}=\mathrm{Ti}, \mathrm{Zr}, \mathrm{Hf} ; \mathrm{M}=\mathrm{Mg}$, Ca, Zn). As the ternary alloys are valence-matched, they exhibit the necessary semiconducting state needed for them to be able to exhibit a piezoelectric response. In particular $\mathrm{Hf}_{x / 2} \mathrm{Mg}_{x / 2} \mathrm{Al}_{1-x} \mathrm{~N}$ is as promising as $\mathrm{Sc}_{x} \mathrm{Al}_{1-x} \mathrm{~N}$ for larger $\mathrm{Al}$ contents where the crystalline quality of wurtzite ScAlN film are found to be reliably high.

These studies also showed a strong positive correlation between the energy difference between the zincblende, a tetrahedrally coordinated phase robust in the calculations, and layered-hexagonal phase in the $\mathrm{TM}_{0.5} \mathrm{M}_{0.5} \mathrm{~N}$ or binary alloying component to AlN and the piezoelectric response of the quarternary or ternary alloy. The alloying components with the largest energy difference produced the alloys with the largest piezoelectric response. This energy difference could therefore be used as a descriptor in large-scale high-throughput searches to identify new 
piezoelectric alloys with strong piezoelectric responses.

Based on my studies of the quarternary alloys, I was inspired to also investigate the little explored $\mathrm{TM}_{0.5} \mathrm{Zn}_{0.5} \mathrm{~N}$ systems. Here, I discovered new ordered piezoelectric $\mathrm{TMZnN}_{2}$ phases with large piezoelectric responses, in $\mathrm{TiZnN}_{2}$ an ordered wurtzite superstructure, and in $\mathrm{ZrZnN}_{2}$ and $\mathrm{HfZnN}_{2}$ a layered structure with alternating octahedral $(\mathrm{Zr}, \mathrm{Hf}) \mathrm{N}$ and tetrahedral $\mathrm{ZnN}$ layers. Moreover, this investigation also revealed a large difference in piezoelectric response of the ordered wurtzite and the disordered wurtzite $\mathrm{TiZnN}_{2}$ phases. The piezoelectric response of the disordered wurtzite is substantially larger than the ordered wurtzite. This is likely caused by a flatter energy landscape in the disordered alloy.

\subsection{Phase stability}

My calculations revealed that the mixing enthalpies of $\mathrm{Y}_{x} \mathrm{Al}_{1-x} \mathrm{~N}$ and $\mathrm{Y}_{x} \mathrm{In}_{1-x} \mathrm{~N}$ are similar to that of the well researched $\mathrm{Sc}_{x} \mathrm{Al}_{1-x} \mathrm{~N}$. The mixing enthalpies strongly indicate that synthesis of the wurtzite phase in these materials is likely possible in similar terms as ScAlN. The crossing point between wurtzite and rocksalt phases in $\mathrm{Y}_{x} \mathrm{Al}_{1-x} \mathrm{~N}$ is at around $75 \% \mathrm{Y}$. However, the magnitudes of the positive mixing enthalpies are substantially higher, corresponding to a stronger driving force towards phase separation. As our experiments show very low crystalline quality already at $x=0.22$, we conclude that in this case it is the strong driving force for phase separation creating a nano-crystalline or amorphous film that is the limiting factor for metastable wurtzite growth, rather than direct competition from a rocksalt alloy phase. In contrast, the $\mathrm{Y}_{x} \operatorname{In}_{1-x} \mathrm{~N}$ crossing point is at $50 \% \mathrm{Y}$, and the mixing enthalpies are even lower than for $\mathrm{Sc}_{x} \mathrm{Al}_{1-x} \mathrm{~N}$. In addition, we show with experiments that it is possible to synthesize this material with up to $51 \% \mathrm{Y}$. Although the crystalline quality of the films deteriorated quickly after $14 \%$, it is not because of a strong driving force towards phase separation into InN and YN according to the mixing enthalpies.

The mixing enthalpies of quarternary $\mathrm{TM}_{x / 2} \mathrm{M}_{x / 2} \mathrm{Al}_{1-x} \mathrm{~N}$ ( $\mathrm{TM}=\mathrm{Ti}, \mathrm{Zr}, \mathrm{Hf}$; $\mathrm{M}=\mathrm{Mg}, \mathrm{Ca}, \mathrm{Zn}$ ) nitride alloys show that these materials have positive mixing enthalpies in similar range as in $\mathrm{Sc}_{x} \mathrm{Al}_{1-x} \mathrm{~N}$, indicating that it is likely that they can be synthesized as metastable alloys in a similar way. The $\mathrm{Ti}_{x / 2} \mathrm{Zn}_{x / 2} \mathrm{Al}_{1-x} \mathrm{~N}$ alloys stand out from the others with a wurtzite phase that is lowest in energy through the entire composition range, with a for these systems comparable low driving force for phase separation.

In addition to the aforementioned ternary and quarternary alloys, I have also predicted the piezoelectric TiZnN $2, \mathrm{ZrZnN}_{2}$, and $\mathrm{HfZnN}_{2}$ phases to be thermodynamically stable. I did this by determining the phase stability at $0 \mathrm{~K}$ and $0 \mathrm{GPa}$ through a high-throughput investigation of possible competing ternary phases in the relatively unexplored ternary systems. Although this does not necessarily mean that the compounds are stable at room temperature or at PVD growth or bulk sintering temperatures, it is a strong enough indication to inspire experimental attempts.

I have also studied the effect of in-plane stresses on the crystalline quality in 
$\mathrm{Sc}_{x} \mathrm{Al}_{1-x} \mathrm{~N} / \mathrm{In}_{y} \mathrm{Al}_{1-y} \mathrm{~N}$ superlattices. Experimentally we observed that crystalline quality of the wurtzite $\mathrm{Sc}_{x} \mathrm{Al}_{1-x} \mathrm{~N}$ with relatively high $\mathrm{Sc}$ content was improved when the $\mathrm{In}_{y} \mathrm{Al}_{1-y} \mathrm{~N}$ layers contain more large In-atoms. We explained this finding using theoretical calculations, showing that the mixing enthalpies for static inplane lattice constants and therefore the energy gain by demixing is reduced as the in-plane lattice parameter is increased.

\subsection{Surface Diffusion}

My contributions to the field of surface diffusion is on the effect of alloying on adatom mobilities on TiN and $\mathrm{Ti}_{0.5} \mathrm{Al}_{0.5} \mathrm{~N}$ surfaces. By investigating the adatom surface energetics for $\mathrm{Ti}$ and $\mathrm{Al}$ on disordered $\mathrm{Ti}_{0.5} \mathrm{Al}_{0.5} \mathrm{~N}(001)$ and comparing it to pure $\mathrm{TiN}\left(\begin{array}{ll}0 & 0\end{array}\right)$, we determine that Ti adatom mobility is dramatically reduced, in contrast to $\mathrm{Al}$ adatoms for which the mobilities are nearly the same on both surfaces.

In order to differentiate the chemical and configurational effect of the $\mathrm{Al}$ introduction in TiN, I investigated the diffusivity of $\mathrm{Ti}, \mathrm{Al}$, and $\mathrm{N}$ adatoms on TiN(lo 01 1), TiN(1 10$)$, TiN(1 11 1) surfaces with and without an Al atom in the surface layer. This study revealed that the reason behind the reduction in Ti mobility is the introduction of $\mathrm{Ti}$ trapping sites in the fourfold hollow positions near the $\mathrm{Al}$ surface atom. In contrast, neither $\mathrm{Al}$ nor $\mathrm{N}$ adatoms show as distinct energy differences. Furthermore, this study also revealed differences in adatom energetics on $\mathrm{TiN}(110)$ and $\mathrm{TiN}(111)$ surfaces. In general, adatom mobilities are fastest on the $\operatorname{TiN}\left(\begin{array}{lll}0 & 0 & 1\end{array}\right)$ surface, slower on the $\operatorname{TiN}\left(\begin{array}{lll}1 & 1 & 1\end{array}\right)$, and slowest on the $\operatorname{TiN}\left(\begin{array}{lll}0 & 1 & 1\end{array}\right)$ surface.

My research also showed that it can be important to consider that the adatoms can be spin-polarized at certain binding positions, but not necessarily all positions. For example, the binding energy of $\mathrm{Ti}$ adatoms on $\mathrm{TiN}(001)$ bulk sites is severely underestimated when the electron spin is disregarded. A Ti adatom is much more likely to stay at a bulk site when spin is included, than without. 


\section{CHAPTER 9}

Outlook

Although my research has answered many questions, it has also inspired new ones. The following sections contain outlooks in the respective areas of my research.

\subsection{Piezoelectric nitrides}

Motivated by the preference for $c$-oriented growth of piezoelectric thin films, my work has been focused mainly on specific piezoelectric and elastic tensorial elements: $e_{33}, C_{33}$, and the clamped piezoelectric response $d_{33, f}$. While the clamped response is not the true $d_{33}$, it is seemingly in better agreement with experiments than the true $d_{33}$. It would, therefore, be interesting to investigate the extent of the clamping effect with respect to layer thickness, or if it is something else than clamped geometry effects that cause the discrepancies.

In addition, it would be possible to further investigate the piezoelectric response as function of configuration, tracing the order-disorder transition with supercells and experiments. Another possibility is to investigate the piezoelectric response in superlattices, novel superstructures, or two-dimensional materials.

\subsection{Phase stability}

My study of $\mathrm{TiZnN} \mathrm{Zn}_{2}, \mathrm{ZrZnN}_{2}$, and $\mathrm{HfZnN}_{2}$ has revealed that they have interesting piezoelectric properties and more importantly that they are stable, at least, at $0 \mathrm{~K}$. The next step is, therefore, to attempt to synthesize these materials experimentally. Furthermore, studying the other stable phases that were discovered during the phase stability study could reveal if they have any potential applications.

The investigation of the effect of clamped in-plane lattice parameters could be extended to other superlattices, or it could be directed towards the prediction 
of which substrates would be optimal for thin film synthesis of certain phases at specific compositions.

\subsection{Surface diffusion}

As the next step for TiN diffusion it would be fascinating to investigating multiple $\mathrm{Al}_{T i}$ substitutions, and to study diffusion on other surfaces, such as the piezoelectric materials that are part of my other studies.

The effect of magnetism could be investigated further with experiments or ab initio MD.

The diffusion barriers that I have obtained could be used to model the kinetics with $\mathrm{KMC}$, and the potentials energy surfaces could be used to modify CMD potentials. It is also possible to lead further method development for surface diffusion on disordered surfaces. 


\section{Bibliography}

1. McPherron, S. P. et al. Evidence for stone-tool-assisted consumption of animal tissues before 3.39 million years ago at Dikika, Ethiopia. Nature 466, 857-60 (2010).

2. Hummel, R. E. Understanding Materials Science: History, Properties, Applications, Second Edition 2nd (Springer, 2004).

3. Wang, Z. L. Towards Self-Powered Nanosystems: From Nanogenerators to Nanopiezotronics. Adv. Funct. Mater. 18, 3553-3567 (2008).

4. Hajati, A. \& Kim, S.-G. Ultra-wide bandwidth piezoelectric energy harvesting. Appl. Phys. Lett. 99, 083105 (2011).

5. Hashimoto, K., Sato, S., Teshigahara, A., Nakamura, T. \& Kano, K. Highperformance surface acoustic wave resonators in the 1 to $3 \mathrm{GHz}$ range using a ScAlN/6H-SiC structure. IEEE Trans. Ultrason. Ferroelectr. Freq. Control 60, 637-42 (2013).

6. Umeda, K. et al. Piezoelectric properties of ScAlN thin films for piezoMEMS devices in 2013 IEEE 26th Int. Conf. Micro Electro Mech. Syst. (IEEE, 2013), 733-736.

7. Suzuki, M., Yanagitani, T. \& Odagawa, H. Polarity-inverted ScAlN film growth by ion beam irradiation and application to overtone acoustic wave (000-1)/(0001) film resonators. Appl. Phys. Lett. 104, 172905 (2014).

8. Jehn, H. A., Hofman, S., Rückborn, V.-E. \& Münz, W.-D. Morphology and properties of sputtered $(\mathrm{Ti}, \mathrm{Al}) \mathrm{N}$ layers on high speed steel substrates as a function of deposition temperature and sputtering atmosphere. J. Vac. Sci. Technol. A Vacuum, Surfaces, Film. 4, 2701 (1986).

9. Munz, W.-D. Titanium aluminum nitride films: A new alternative to TiN coatings. J. Vac. Sci. Technol. A Vacuum, Surfaces, Film. 4, 2717 (1986). 
10. Knotek, O., Böhmer, M \& Leyendecker, T. On structure and properties of sputtered Ti and Al based hard compound films. J. Vac. Sci. Technol. A Vacuum, Surfaces, Film. 4, 2695 (1986).

11. Akiyama, M. et al. Enhancement of Piezoelectric Response in Scandium Aluminum Nitride Alloy Thin Films Prepared by Dual Reactive Cosputtering. Adv. Mater. 21, 593-596 (2009).

12. Chen, Q. \& Sundman, B. Thermodynamic Assessment of the Ti-Al-N System. J. Phase Equilibria 19, 146-160 (1998).

13. Schuster, J. \& Bauer, J. The ternary systems Sc-Al-N and Y-Al-N. J. Less Common Met. 109, 345-350 (1985).

14. Höglund, C. et al. Effects of volume mismatch and electronic structure on the decomposition of ScAlN and TiAlN solid solutions. Phys. Rev. B 81, 224101 (2010).

15. Antczak, G. \& Ehrlich, G. Surface Diffusion: Metals, Metal Atoms, and Clusters First, 784 (Cambridge University Press, Cambridge, 2010).

16. Gall, D, Kodambaka, S, Wall, M. A., Petrov, I \& Greene, J. E. Pathways of atomistic processes on TiN (001) and (111) surfaces during film growth: an ab initio study. J. Appl. Phys. 93, 9086-9094 (2003).

17. Born, M. \& Oppenheimer, R. Zur Quantentheorie der Molekeln. Ann. Phys. 389, 457-484 (1927).

18. Bloch, F. Über die Quantenmechanik der Elektronen in Kristallgittern. Zeitschrift für Phys. 52, 555-600 (1929).

19. Thomas, L. H. The calculation of atomic fields. Math. Proc. Cambridge Philos. Soc. 23, 542 (1927).

20. Fermi, E. Un metodo statistico per la determinazione di alcune priorieta dellatome. Rend. Accad. Naz. Lincei 6, 32 (1927).

21. Hohenberg, P. \& Kohn, W. Inhomogeneous Electron Gas. Phys. Rev. 136, B864-B871 (1964).

22. Martin, R. M. Electronic Structure: Basic Theory and Practical Methods (Cambridge University Press, 2004).

23. Kohn, W. \& Sham, L. J. Self-Consistent Equations Including Exchange and Correlation Effects. Phys. Rev. 140, A1133-A1138 (1965).

24. Kohn, W. Nobel Lecture: Electronic structure of matterwave functions and density functionals. Rev. Mod. Phys. 71, 1253-1266 (1999).

25. Von Barth, U \& Hedin, L. A local exchange-correlation potential for the spin polarized case. i. J. Phys. C Solid State Phys. 5, 1629-1642 (1972).

26. Ceperley, D. \& Alder, B. Ground State of the Electron Gas by a Stochastic Method. Phys. Rev. Lett. 45 (1980).

27. Vosko, S. H., Wilk, L. \& Nusair, M. Accurate spin-dependent electron liquid correlation energies for local spin density calculations: a critical analysis. Can. J. Phys. 58, 1200-1211 (1980). 
28. Perdew, J. P. \& Zunger, A. Self-interaction correction to density-functional approximations for many-electron systems. Phys. Rev. B 23, 5048 (1981).

29. Becke, A. Density-functional exchange-energy approximation with correct asymptotic behavior. Phys. Rev. A 38, 3098-3100 (1988).

30. Perdew, J. \& Wang, Y. Accurate and simple analytic representation of the electron-gas correlation energy. Phys. Rev. B 45, 244-249 (1992).

31. Perdew, J. P., Burke, K. \& Ernzerhof, M. Generalized gradient approximation made simple. Phys. Rev. Lett. 77, 3865-3868 (1996).

32. Hammer, B., Hansen, L. \& Nørskov, J. Improved adsorption energetics within density-functional theory using revised Perdew-Burke-Ernzerhof functionals. Phys. Rev. B 59, 7413-7421 (1999).

33. Perdew, J. P., Burke, K \& Ernzerhof, M. Erratum: Generalized Gradient Approximation Made Simple. Phys. Rev. Lett. 78, 1396 (1997).

34. Anisimov, V., Zaanen, J. \& Andersen, O. Band theory and Mott insulators: Hubbard U instead of Stoner I. Phys. Rev. B 44 (1991).

35. Anisimov, V., Solovyev, I. \& Korotin, M. Density-functional theory and NiO photoemission spectra. Phys. Rev. B 48 (1993).

36. Anisimov, V., Aryasetiawan, F \& Lichtenstein, A. First-principles calculations of the electronic structure and spectra of strongly correlated systems: the LDA + U method. J. Phys. Condens. Matter 9, 767 (1997).

37. Lee, C., Yang, W \& Parr, R. Development of the Colic-Salvetti correlationenergy formula into a functional of the electron density. Phys. Rev. B 37 (1988).

38. Schimka, L et al. Accurate surface and adsorption energies from many-body perturbation theory. Nat. Mater. 9, 741-744 (2010).

39. Vanderbilt, D. Soft self-consistent pseudopotentials in a generalized eigenvalue formalism. Phys. Rev. B 41, 7892 (1990).

40. Blöchl, P. E. Projector augmented-wave method. Phys. Rev. B 50, 1795317979 (1994).

41. Kresse, G. \& Joubert, D. From ultrasoft pseudopotentials to the projector augmented-wave method. Phys. Rev. B 59, 1758 (1999).

42. Ruban, A. V. \& Abrikosov, I. A. Configurational thermodynamics of alloys from first principles: effective cluster interactions. Reports Prog. Phys. 71, 046501 (2008).

43. Zunger, A, Wei, S. H., Ferreira, L. G. \& Bernard, J. E. Special quasirandom structures. Phys. Rev. Lett. 65, 353-356 (1990).

44. Sanchez, J. M., Ducastelle, F. \& Gratias, D. Generalized cluster description of multicomponent systems. Phys. A Stat. Mech. its Appl. 128, 334-350 (1984).

45. Sanchez, J. Cluster expansions and the configurational energy of alloys. Phys. Rev. B 48, 13-15 (1993). 
46. Tasnádi, F., Odén, M. \& Abrikosov, I. A. Ab initio elastic tensor of cubic $\mathrm{Ti}_{0.5} \mathrm{Al}_{0.5} \mathrm{~N}$ alloys: Dependence of elastic constants on size and shape of the supercell model and their convergence. Phys. Rev. B 85, 144112 (2012).

47. Finnis, M. Interatomic Forces in Condensed Matter (OUP Oxford, 2003).

48. Romanov, A. E., Baker, T. J., Nakamura, S. \& Speck, J. S. Strain-induced polarization in wurtzite III-nitride semipolar layers. J. Appl. Phys. 100, 023522 (2006).

49. Resta, R. Macroscopic polarization in crystalline dielectrics: the geometric phase approach. Rev. Mod. Phys. 66 (1994).

50. Vanderbilt, D. Berry-phase theory of proper piezoelectric response. J. Phys. Chem. Solids 61, 147-151 (2000).

51. Bernardini, F, Fiorentini, V \& Vanderbilt, D. Spontaneous polarization and piezoelectric constants of III-V nitrides. Phys. Rev. B 56, R10024-R10027 (1997).

52. Xiao, D., Chang, M.-C. \& Niu, Q. Berry phase effects on electronic properties. Rev. Mod. Phys. 82, 1959-2007 (2010).

53. Furukawa, T. Piezoelectricity and pyroelectricity in polymers. Electr. Insul. IEEE Trans. 24, 375-394 (1989).

54. Jiang, W., Zhang, R., Jiang, B. \& Cao, W. Characterization of piezoelectric materials with large piezoelectric and electromechanical coupling coefficients. Ultrasonics 41, 55-63 (2003).

55. Dubois, M.-A. \& Muralt, P. Stress and piezoelectric properties of aluminum nitride thin films deposited onto metal electrodes by pulsed direct current reactive sputtering. J. Appl. Phys. 89, 6389 (2001).

56. Caro, M. A. et al. Piezoelectric coefficients and spontaneous polarization of ScAlN. J. Phys. Condens. Matter 27, 245901 (2015).

57. Wu, X., Vanderbilt, D. \& Hamann, D. R. Systematic treatment of displacements, strains, and electric fields in density-functional perturbation theory. Phys. Rev. B - Condens. Matter Mater. Phys. 72, 1-13 (2005).

58. Tasnádi, F. et al. Origin of the Anomalous Piezoelectric Response in Wurtzite $\mathrm{Sc}_{x} \mathrm{Al}_{1-x} \mathrm{~N}$ Alloys. Phys. Rev. Lett. 104, 137601 (2010).

59. Damjanovic, D. A morphotropic phase boundary system based on polarization rotation and polarization extension. Appl. Phys. Lett. 97, 62906 (2010).

60. Lueng, C. M., Chan, H. L. W., Fong, W. K., Surya, C. \& Choy, C. L. Piezoelectric Coefficients of Aluminum Nitride and Gallium Nitride. MRS Proc. 572, 4133-4135 (1999).

61. Sheleg, A. \& Savastenko, V. Determination of elastic-constants of hexagonal crystals from measured values of dynamic atomic displacements. Inorg. Mater. 15, 1257-1261 (1979). 
62. Cao, C., Chan, H. \& Choy, C. Piezoelectric coefficient of InN thin films prepared by magnetron sputtering. Thin Solid Films 441, 287-291 (2003).

63. Alling, B. Metal to semiconductor transition and phase stability of $\mathrm{Ti}_{1-x} \mathrm{Mg}_{x} \mathrm{~N}_{y}$ alloys investigated by first-principles calculations. Phys. Rev. B 89, 085112 (2014).

64. Porter, D. A. \& Easterling, K. E. Phase Transformations in Metals and Alloys, Third Edition (Revised Reprint) (1992).

65. Thore, A., Dahlqvist, M., Alling, B. \& Rosén, J. Temperature dependent phase stability of nanolaminated ternaries from first-principles calculations. Comput. Mater. Sci. 91, 251-257 (2014).

66. Holleck, H. Metastable coatings Prediction of composition and structure. Surf. Coatings Technol. 36, 151-159 (1988).

67. Dahlqvist, M., Alling, B. \& Rosén, J. Stability trends of MAX phases from first principles. Phys. Rev. B 81, 220102 (2010).

68. Ayouchi, R., Casteleiro, C., Santos, L. \& Schwarz, R. RF-plasma assisted PLD growth of Zn3N2 thin films. Phys. Status Solidi 7, 2294-2297 (2010).

69. Wang, L., Maxisch, T. \& Ceder, G. Oxidation energies of transition metal oxides within the GGA + U framework. Phys. Rev. B 73, 195107 (2006).

70. Ohring, M. Materials Science of Thin Films (Elsevier Science, 2001).

71. Greene, J. E., Barnett, S. A., Sundgren, J.-E. \& Rockett, A. in (eds Auciello, O., Gras-Marti, A., Valles-Abarca, J. A. \& Flamm, D. L.) 281-311 (Springer Netherlands, Dordrecht, 1990).

72. Hsiao, C. L. et al. Curved-lattice epitaxial growth of $\operatorname{In}_{x} \mathrm{Al}_{1-x} \mathrm{~N}$ nanospirals with tailored chirality. Nano Lett. 15, 294-300 (2015).

73. Höglund, C. et al. Wurtzite structure $\mathrm{Sc}_{1-x} \mathrm{Al}_{x} \mathrm{~N}$ solid solution films grown by reactive magnetron sputter epitaxy: Structural characterization and firstprinciples calculations. J. Appl. Phys. 107, 123515 (2010).

74. Höglund, C. et al. Cubic $\mathrm{Sc}_{1-x} \mathrm{Al}_{x} \mathrm{~N}$ solid solution thin films deposited by reactive magnetron sputter epitaxy onto $\mathrm{ScN}(111)$. J. Appl. Phys. 105, 1-7 (2009).

75. Rovere, F., Music, D., Schneider, J. M. \& Mayrhofer, P. H. Experimental and computational study on the effect of yttrium on the phase stability of sputtered Cr-Al-Y-N hard coatings. Acta Mater. 58, 2708-2715 (2010).

76. Mayrhofer, P. et al. Microstructure and piezoelectric response of $\mathrm{Y}_{x} \mathrm{Al}_{1-x} \mathrm{~N}$ thin films. Acta Mater. 100, 81-89 (2015).

77. Strite, S, Chandrasekhar, D \& Smith, D. Structural properties of InN films grown on GaAs substrates: observation of the zincblende polytpe. J. Cryst. Growth 127, 204-208 (1993).

78. Bhattacharya, P, Sharma, T. K., Singh, S, Ingale, A \& Kukreja, L. M. Observation of zincblend phase in InN thin films grown on sapphire by nitrogen plasma-assisted pulsed laser deposition. J. Cryst. Growth 236, 5-9 (2002). 
79. Bhuiyan, A. G., Hashimoto, A. \& Yamamoto, A. Indium nitride (InN): A review on growth, characterization, and properties. J. Appl. Phys. 94, 2779 2808 (2003).

80. Yokoyama, T. et al. Effect of $\mathrm{Mg}$ and $\mathrm{Zr}$ co-doping on piezoelectric AlN thin films for bulk acoustic wave resonators. IEEE Trans. Ultrason. Ferroelectr. Freq. Control 61, 1322-1328 (2014).

81. Yokoyama, T. et al. Highly piezoelectric co-doped AlN thin films for wideband FBAR applications in 2014 IEEE Int. Ultrason. Symp. (IEEE, Chicago, 2014), 281-288.

82. Minh, L. V. et al. Piezoelectric micro energy harvesters employing advanced (Mg,Zr)-codoped AIN thin film in 2015 28th IEEE Int. Conf. Micro Electro Mech. Syst. (IEEE, Estoril, 2015), 1094-1097.

83. Minh, L. V. et al. Highly Piezoelectric MgZr Co-Doped Aluminum NitrideBased Vibrational Energy Harvesters. 62, 2005-2008 (2015).

84. Fick, A. Ueber Diffusion. Ann. der Phys. und Chemie 170, 59-86 (1855).

85. Fick, A. On liquid diffusion. Philos. Mag. Ser. 4 10, 30-39 (1855).

86. Heitjans, P \& Kärger, J. Diffusion in Condensed Matter 1st ed. (SpringerVerlag, Berlin/Heidelberg, 2005).

87. Mills, G, Jónsson, H \& Schenter, G. K. Reversible work transition state theory: application to dissociative adsorption of hydrogen. Surf. Sci. 324, 305-337 (1995).

88. Henkelman, G., Uberuaga, B. \& Jónsson, H. A climbing image nudged elastic band method for finding saddle points and minimum energy paths. J. Chem. Phys. 113 (2000).

89. Sheppard, D., Terrell, R. \& Henkelman, G. Nudged elastic band method for finding minimum energy paths of transitions. J. Chem. Phys. 128, 134106 (2008).

90. Vineyard, G. H. Frequency factors and isotope effects in solid state rate processes. J. Phys. Chem. Solids 3, 121-127 (1957).

91. Wrigley, J. D., Twigg, M. E. \& Ehrlich, G. Lattice walks by long jumps. J. Chem. Phys. 93, 2885 (1990).

92. Kolmogoroff, A. Über die analytischen Methoden in der Wahrscheinlichkeitsrechnung. Math. Ann. 104, 415-458 (1931).

93. Leyendecker, T, Lemmer, O, Esser, S \& Ebberink, J. The development of the PVD coating TiAlN as a commercial coating for cutting tools. Surf. Coatings Technol. 48, 175-178 (1991).

94. Subramanian, B, Muraleedharan, C. V., Ananthakumar, R \& Jayachandran, M. A comparative study of titanium nitride (TiN), titanium oxy nitride ( TiON) and titanium aluminum nitride (TiAlN), as surface coatings for bio implants. Surf. Coatings Technol. 205, 5014-5020 (2011). 
95. Beensh-Marchwicka, G., Kròl-Stepniewska, L. \& Posadowski, W. Structure of thin films prepared by the cosputtering of titanium and aluminium or titanium and silicon. Thin Solid Films 82, 313-320 (1981).

96. Knotek, O., Münz, W. D. \& Leyendecker, T. Industrial deposition of binary, ternary, and quaternary nitrides of titanium, zirconium, and aluminum. $J$. Vac. Sci. Technol. A Vacuum, Surfaces, Film. 5, 2173 (1987).

97. Jindal, P. C., Santhanam, A. T., Schleinkofer, U \& Shuster, A. F. Performance of PVD TiN, TiCN, and TiAlN coated cemented carbide tools in turning. Int. J. Refract. Met. Hard Mater. 17, 163-170 (1999).

98. Hörling, A, Hultman, L, Odén, M, Sjölén, J \& Karlsson, L. Mechanical properties and machining performance of $\mathrm{Ti}_{1-x} \mathrm{Al}_{x} \mathrm{~N}$-coated cutting tools. Surf. Coatings Technol. 191, 384-392 (2005).

99. McIntyre, D, Greene, J. E., Hakansson, G, Sundgren, J. E. \& Munz, W. D. Oxidation of metastable single-phase polycrystalline $\mathrm{Ti}_{0.5} \mathrm{Al}_{0.5} \mathrm{~N}$ films: Kinetics and mechanisms. J. Appl. Phys. 67, 1542 (1990).

100. Chim, Y. C., Ding, X. Z., Zeng, X. T. \& Zhang, S. Oxidation resistance of TiN, CrN, TiAlN and CrAlN coatings deposited by lateral rotating cathode arc. Thin Solid Films 517, 4845-4849 (2009).

101. Music, D \& Schneider, J. M. Ab initio study of $\mathrm{Ti}_{0.5} \mathrm{Al}_{0.5} \mathrm{~N}(001)$ residual and environmental gas interactions. New J. Phys. 15, 073004 (2013).

102. Mayrhofer, P. H. et al. Self-organized nanostructures in the TiAlN system. Appl. Phys. Lett. 83, 2049 (2003).

103. Santana, A. E., Karimi, A., Derflinger, V. H., Schütze, A. \& Schu, A. Thermal treatment effects on microstructure and mechanical properties of TiAlN thin films. Tribol. Lett. 17, 689-696 (2004).

104. Rachbauer, $\mathrm{R}$ et al. Decomposition pathways in age hardening of Ti-Al-N films. J. Appl. Phys. 110, 023515 (2011).

105. Johnson, L., Thuvander, M., Stiller, K., Odén, M. \& Hultman, L. Spinodal decomposition of $\mathrm{Ti}_{0.33} \mathrm{Al}_{0.67} \mathrm{~N}$ thin films studied by atom probe tomography. Thin Solid Films 520, 4362-4368 (2012).

106. Knutsson, A. et al. Microstructure evolution during the isostructural decomposition of TiAlN - A combined in-situ small angle x-ray scattering and phase field study. J. Appl. Phys. 113, 213518 (2013).

107. Knutsson, A., Schramm, I. C., Asp Grönhagen, K., Mücklich, F. \& Odén, M. Surface directed spinodal decomposition at TiAlN/TiN interfaces. J. Appl. Phys. 113, 114305 (2013).

108. Alling, B. et al. Mixing and decomposition thermodynamics of $c-\mathrm{Ti}_{1-x} \mathrm{Al}_{x} \mathrm{~N}$ from first-principles calculations. Phys. Rev. B 75, 045123 (2007).

109. Alling, B., Karimi, A. \& Abrikosov, I. A. Electronic origin of the isostructural decomposition in cubic $\mathrm{M}_{1-x} \mathrm{Al}_{x} \mathrm{~N}(\mathrm{M}=\mathrm{Ti}, \mathrm{Cr}, \mathrm{Sc}, \mathrm{Hf})$ : A first-principles study. Surf. Coatings Technol. 203, 883-886 (2008). 
110. Alling, B., Odén, M., Hultman, L. \& Abrikosov, I. A. Pressure enhancement of the isostructural cubic decomposition in $\mathrm{Ti}_{1-x} \mathrm{Al}_{x} \mathrm{~N}$. Appl. Phys. Lett. 95, 181906 (2009).

111. Eyring, H. The Activated Complex in Chemical Reactions. J. Chem. Phys. 3, 107 (1935).

112. Bogicevic, A., Strömquist, J. \& Lundqvist, B. I. Low-Symmetry Diffusion Barriers in Homoepitaxial Growth of Al(111). Phys. Rev. Lett. 81, 637-640 (1998).

113. Mantina, M. et al. First-Principles Calculation of Self-Diffusion Coefficients. Phys. Rev. Lett. 100, 215901 (2008).

114. Ruberto, C., Vojvodic, A. \& Lundqvist, B. I. Nature of versatile chemisorption on $\mathrm{TiC}(111)$ and $\mathrm{TiN}(111)$ surfaces. Solid State Commun. 141, 48-52 (2007).

115. Ruberto, C. \& Lundqvist, B. I. Nature of adsorption on TiC(111) investigated with density-functional calculations. Phys. Rev. B 75, 235438 (2007).

116. Ciobanu, C. V., Tambe, D. T. \& Shenoy, V. B. First-principles calculations of step formation energies and step interactions on TiN(001). Surf. Sci. 582, 145-150 (2005).

117. Graciani, J, Fdez Sanz, J, Asaki, T, Nakamura, K \& Rodriguez, J. A. Interaction of oxygen with $\mathrm{TiN}(001)$ : NO exchange and oxidation process. $J$. Chem. Phys. 126, 244713-244718 (2007).

118. Sanyal, S., Waghmare, U. V. \& Ruud, J. A. Adsorption of water on TiN (100), (110) and (111) surfaces: A first-principles study. Appl. Surf. Sci. 257, 6462-6467 (2011).

119. Akiyama, T., Nakamura, K. \& Ito, T. Ab initio-based study for adatom kinetics on $\mathrm{AlN}(0001)$ surfaces during metal-organic vapor-phase epitaxy growth. Appl. Phys. Lett. 100, 251601 (2012).

120. Sangiovanni, D. G. et al. Ab initio and classical molecular dynamics simulations of $\mathrm{N}_{2}$ desorption from TiN(001) surfaces. Surf. Sci. 624, 25-31 (2014).

121. Greene, J. E., Sundgren, J.-E., Hultman, L., Petrov, I. \& Bergstrom, D. B. Development of preferred orientation in polycrystalline TiN layers grown by ultrahigh vacuum reactive magnetron sputtering. Appl. Phys. Lett. 67, 2928 (1995).

122. Hultman, L., Sundgren, J.-E., Greene, J. E., Bergstrom, D. B. \& Petrov, I. High-flux low-energy $(\cong 20 \mathrm{eV}) \mathrm{N}_{2}^{+}$ion irradiation during TiN deposition by reactive magnetron sputtering: Effects on microstructure and preferred orientation. J. Appl. Phys. 78, 5395-5403 (1995).

123. Beckers, M, Schell, N, Martins, R. M. S., Mucklich, A \& Moller, W. In situ $\mathrm{x}$-ray diffraction studies concerning the influence of $\mathrm{Al}$ concentration on the texture development during sputter deposition of TiAlN thin films. J. Vac. Sci. Technol. A Vacuum, Surfaces, Film. 23, 1384 (2005). 
124. Tholander, C. Ab initio piezoelectricity and energy landscape of $Y_{0.375} A l_{0.626} N$ 2011. 
[I] Volume matching condition to establish the enhanced piezoelectricity in ternary $(\mathrm{Sc}, \mathrm{Y})_{0.5}(\mathrm{Al}, \mathrm{Ga}, \mathrm{In})_{0.5} \mathrm{~N}$ alloys

C. Tholander, I.A. Abrikosov, L. Hultman, and F. Tasnádi Physical Review B, 87, 94107 (2013).

[II] $\mathbf{Y}_{x} \mathbf{A l}_{1-x} \mathbf{N}$ thin films

A. Žukauskaitè, C. Tholander, J. Palisaitis, P.O.A. Persson, V. Darakchieva, N. Ben Sedrine, F. Tasnádi, B. Alling, J. Birch, and L. Hultman Journal of Physics D: Applied Physics, 45, 422001 (2012).

[III] Ab initio calculations and experimental study of piezoelectric $\mathbf{Y}_{x} \mathbf{I n}_{1-x} \mathbf{N}$ thin films deposited using reactive magnetron sputter epitaxy C. Tholander, J. Birch, F. Tasnádi, L. Hultman, J. Palisaitis, P.O.Å. Persson, J. Jensen, P. Sandström, B. Alling, and A. Žukauskaitè Acta Materialia, 105, 199-206, (2016).

[IV] Stabilization of wurtzite $\mathrm{Sc}_{0.4} \mathrm{Al}_{0.6} \mathbf{N}$ in pseudomorphic epitaxial $\mathbf{S c}_{x} \mathbf{A l}_{1-x} \mathbf{N} / \mathbf{I n}_{y} \mathbf{A l}_{1-y} \mathbf{N}$ superlattices

A. Žukauskaite, C. Tholander, F. Tasnádi, B. Alling, J. Palisaitis, P.O.Å. Persson, L. Hultman, and J. Birch Acta Materialia, 94, 101-110 (2015). 
[V] Large piezoelectric response of quarternary wurtzite nitride alloys and its physical origin from first principles

C. Tholander, F. Tasnádi, I.A. Abrikosov, L. Hultman, J. Birch, and B. Alling Physical Review B, 92, 174119, (2015).

[VI] Strong piezoelectric response in stable $\mathbf{T i Z n N}, \mathbf{Z r Z n N}_{2}$ and $\mathrm{HfZnN}_{2}$ found by $a b$ initio high-throughput approach

C. Tholander, C. B. A. Andersson, R. Armiento, F. Tasnádi, and B. Alling In manuscript, (2016).

[VII] Configurational disorder effects on adatom mobilities on $\mathbf{T i}_{1-x} \mathbf{A l}_{x} \mathbf{N}\left(\begin{array}{lll}0 & 0 & 1\end{array}\right)$ surfaces from first principles

B. Alling, P. Steneteg, C. Tholander, F. Tasnádi, I. Petrov, J.E. Greene, and L. Hultman

Physical Review B, 85, 245422 (2012).

[VIII] Effect of Al substitution on $\mathrm{Ti}, \mathrm{Al}$, and $\mathbf{N}$ adatom dynamics on TiN(0 01 ), (011), and (111) surfaces

C. Tholander, B. Alling, F. Tasnádi, J.E. Greene, and L. Hultman

Surface Science, 630, 28 (2014). 


\section{Summary of included papers}

\section{Paper I}

Volume matching condition to establish the enhanced piezoelectricity in ternary $(\mathrm{Sc}, \mathrm{Y})_{0.5}(\mathrm{Al}, \mathrm{Ga}, \mathrm{In})_{0.5} \mathrm{~N}$ alloys

\section{Summary}

This work is a continuation of the work presented in my Master's thesis ${ }^{1}$. Here, we performed a search for new candidates for high temperature piezoelectric devices, guided by the giant piezoelectric increase found in ScAlN. I conclude that volume matching of the binary parent compounds is a key part in maximizing the increase of the piezoelectric response.

\section{Author's contribution}

For this work, I took part in planning the project, performed all of the calculations, took part in the discussion, and wrote the majority of the manuscript.

\section{Paper II}

$\mathbf{Y}_{x} \mathbf{A l}_{1-x} \mathbf{N}$ thin films

\section{Summary}

An experimental and theoretical investigation of $\mathrm{Y}_{x} \mathrm{Al}_{1-x} \mathrm{~N}$ thin films. The theoretical work include $\mathrm{Y}_{x} \mathrm{Al}_{1-x} \mathrm{~N}$ lattice parameters, and phase stability calculations for rocksalt, layered-hexagonal, and wurtzite phases in the range $0 \leq x \leq 1$. We show that it is possible to synthesize the wurtzite structure, both with theory and experiment.

\footnotetext{
${ }^{1}$ Ref. [124]
} 


\title{
Author's contribution
}

I performed all of the theoretical work, took part in discussion of the results, and wrote the parts in the manuscript relating to the theoretical calculations.

\section{Paper III}

\begin{abstract}
Ab initio calculations and experimental study of piezoelectric $\mathbf{Y}_{x} \operatorname{In}_{1-x} \mathbf{N}$ thin films deposited using reactive magnetron sputter epitaxy
\end{abstract}

\section{Summary}

An experimental and theoretical investigation of $\mathrm{Y}_{x} \operatorname{In}_{1-x} \mathrm{~N}$ thin films. The theoretical work include $\mathrm{Y}_{x} \operatorname{In}_{1-x} \mathrm{~N}$ lattice parameters, and phase stability calculations for rocksalt, zincblende crystal structure, layered-hexagonal, and wurtzite in the range $0 \leq x \leq 1$. We show that it is possible to synthesize the wurtzite structure, both with theory and experiment. In addition, the piezoelectric properties are investigated theoretically and experimentally.

\section{Author's contribution}

I took part in planning the study, performed all of the theoretical work, took part in discussion of the results, and wrote the parts in the manuscript relating to the theoretical calculations.

\section{Paper IV}

\section{Stabilization of wurtzite $\mathrm{Sc}_{0.4} \mathrm{Al}_{0.6} \mathrm{~N}$ in pseudomorphic epitaxial} $\mathbf{S c}_{x} \mathbf{A l}_{1-x} \mathbf{N} / \mathbf{I n}_{y} \mathbf{A l}_{1-y} \mathbf{N}$ superlattices

\section{Summary}

An experimental and theoretical investigation of wurtzite $\mathrm{Sc}_{0.4} \mathrm{Al}_{0.6} \mathrm{~N}$ in pseudomorphic epitaxial $\mathrm{Sc}_{x} \mathrm{Al}_{1-x} \mathrm{~N} / \mathrm{In}_{y} \mathrm{Al}_{1-y} \mathrm{~N}$ superlattices. Experimentally we demonstrate the effects of lattice mismatch and the resulting stresses on formation and stability of metastable wurtzite films. Theory is used to explain the increased stability from layers with larger in-plane lattice parameter with mixing enthalpies for fixed in-plane lattice parameters.

\section{Author's contribution}

I performed all of the theoretical work, took part in discussion of the results, and wrote the parts related to the theoretical calculations. 


\section{Paper V}

Large piezoelectric response of quarternary wurtzite nitride alloys and its physical origin from first principles

\section{Summary}

A theoretical investigation of quarternary piezoelectric wurtzite nitrides. We study the stability of the alloys using mixing enthalpies. We determine the most promising piezoelectric alloys and propose a descriptor that can be used in future largescale high-throughput searches for new piezoelectric responses.

\section{Author's contribution}

I took part in planning the study, performed all of the theoretical work, took part in discussion of the results, and wrote the majority of the manuscript.

\section{Paper VI}

Strong piezoelectric response in stable $\mathrm{TiZnN} \mathbf{n N}_{2}, \mathrm{ZrZnN}_{2}$ and $\mathrm{HfZnN}_{2}$ found by $a b$ initio high-throughput approach

\section{Summary}

A theoretical study of piezoelectric $\mathrm{TMZnN}_{2}(\mathrm{TM}=\mathrm{Ti}, \mathrm{Zr}, \mathrm{Hf})$ structures. These materials are predicted stable from calculated zero temperature and zero pressure phase diagrams, obtained using large-scale high-throughput calculations.

\section{Author's contribution}

I took part in planning the study, performed the majority of the calculations, took part in discussion of the results, and wrote the manuscript. 


\section{Paper VII}

Configurational disorder effects on adatom mobilities on $\mathbf{T i}_{1-x} \mathbf{A l}_{x} \mathbf{N}\left(\begin{array}{lll}0 & 0 & 1\end{array}\right)$ surfaces from first principles

\section{Summary}

This paper, is a study of the configurational disorder effects on adatom mobilities on $\mathrm{Ti}_{1-x} \mathrm{Al}_{x} \mathrm{~N}\left(\begin{array}{lll}0 & 0 & 1\end{array}\right)$ surface by comparing the potential energy landscapes of $\mathrm{Ti}$ and $\mathrm{Al}$ adsorption on these surfaces. We conclude that the disorder reduce the $\mathrm{Ti}$ mobility, while Al mobilities experience only small reductions.

\section{Author's contribution}

For this work, I performed convergence tests of the structures used, and also took part in the discussion of the results.

\section{Paper VIII}

Effect of Al substitution on $\mathbf{T i}, \mathbf{A l}$, and $\mathbf{N}$ adatom dynamics on $\mathbf{T i N}\left(\begin{array}{lll}0 & 0 & 1\end{array}\right)$, (0 1 11$)$, and (111) surfaces

\section{Summary}

In this article, I used DFT to investigate the effect of $\mathrm{Al}$ substitution on $\mathrm{Ti}, \mathrm{Al}$, and $\mathrm{N}$ adatom dynamics on $\mathrm{TiN}\left(\begin{array}{lll}0 & 0 & 1\end{array}\right),\left(\begin{array}{lll}0 & 1 & 1\end{array}\right)$, and (11 11 ) surfaces. It is found that in general adatom mobilities are fastest on $\operatorname{TiN}\left(\begin{array}{ll}0 & 01)\end{array}\right)$, slower on $\operatorname{TiN}(111)$, and slowest on $\mathrm{TiN}(011)$. We confirm that the $\mathrm{Al}$ substitution on $\mathrm{TiN}(001)$ reduce the $\mathrm{Ti}$ migration rate with little reduction to the $\mathrm{Al}$ migration. The effect was also found to be the opposite on $\operatorname{TiN}(111)$. In addition, we show that magnetic effects have a significant impact on the Ti adatom binding energies and diffusion path.

\section{Author's contribution}

I took part in planning of this project, performed the calculations, participated in the evaluation and interpretation of the results, and was responsible for writing the manuscript. 


\section{Papers}

The articles associated with this thesis have been removed for copyright reasons. For more details about these see:

http://urn.kb.se/resolve?urn=urn:nbn:se:liu:diva- 125919 


\section{APPENDIX A}

\section{Periodic table}

The work in this thesis covers many different materials and alloys. This periodic table is intended to be a guide to the relations between them.

The data in the table has been updated according to the latest data from the International Union of Pure and Applied Chemistry (IUPAC) at the time of print. ${ }^{1}$

\footnotetext{
${ }^{1}$ The names of elements $113,115,117$, and 118 are guesses based upon possible names assembled in a list by The Sceptical Chymist, a Nature Chemistry blog. http://blogs.nature.com/thescepticalchymist/2016/01/new-kids-on-the-p-block.html
} 


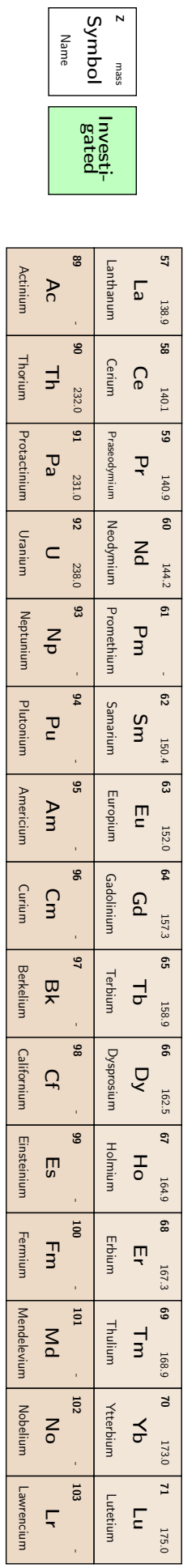

\begin{tabular}{|c|c|c|c|c|c|c|}
\hline 苞. & 总 & 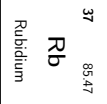 & 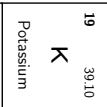 & 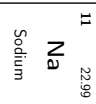 & ᄃ. & 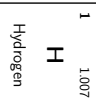 \\
\hline 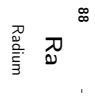 & 啀 & 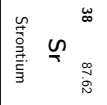 & 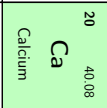 & 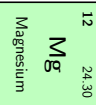 & 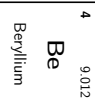 & $\stackrel{N}{\overline{\bar{E}}}$ \\
\hline 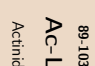 & 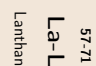 & $\prec^{\text {品 }}$ & $\sum^{n} n^{n}$ & $\stackrel{\omega}{=}$ & & \\
\hline
\end{tabular}

\begin{tabular}{|c|c|c|c|}
\hline$\prod_{n}^{\vec{s}}$ & 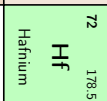 & 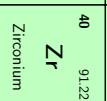 & 青 \\
\hline$\sigma^{\vec{\sigma}}$ & 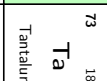 & $z_{0} z_{0}^{=}$ & 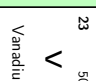 \\
\hline
\end{tabular}

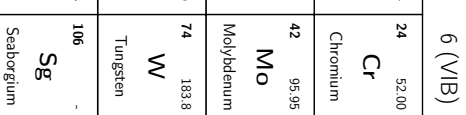

0
$\frac{0}{2}$
$\frac{0}{0}$
0

\begin{tabular}{|c|c|c|c|}
\hline 点 & गृ ज & $\overrightarrow{0} \quad$ \# & $\frac{z}{N} \quad \tilde{n}$ \\
\hline$\frac{\omega}{\sigma}$ & 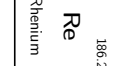 & 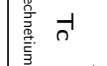 & \\
\hline
\end{tabular}

$\frac{0}{\frac{0}{0}}$

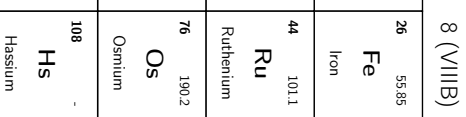

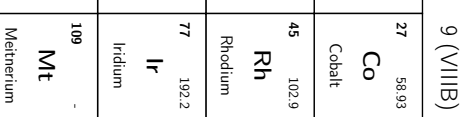

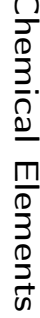

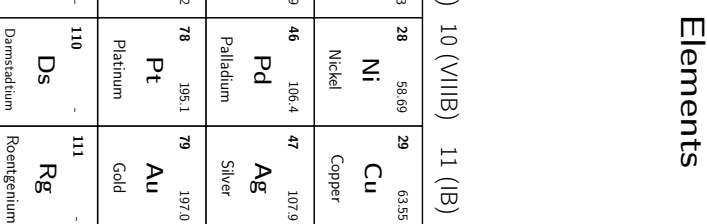

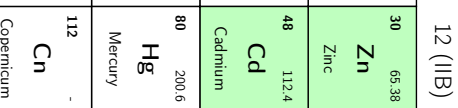

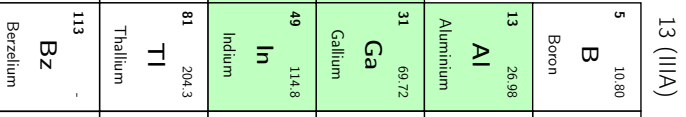

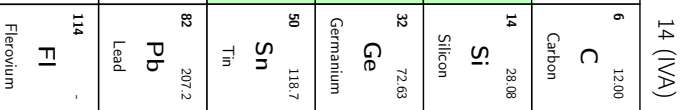

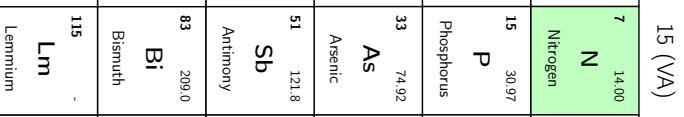

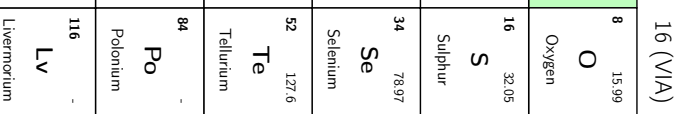

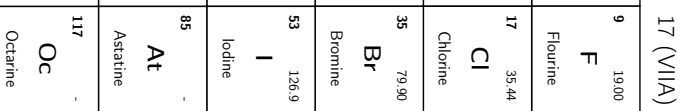

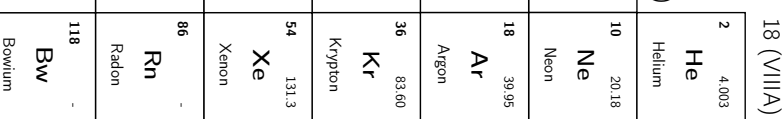




\section{APPENDIX B}

\section{Glossary and acronyms}

\section{Glossary}

adatom

Atom adsorbed on surface

\section{admolecule}

Molecule adsorbed on surface

\section{alloy}

A mix of metals or a metal and non-metal elements

binary alloy

Alloy consisting of two elements

\section{chemical vapor deposition}

Common technique used to synthesize thin solid films

\section{convex hull}

Mathematical construction, here used to mark stable phases and regions where phase separation will occur

\section{first principles}

Theoretical work that is based on quantum mechanics without empirical assumptions, also called ab initio 
grain

Crystal subvolume with a specific internal crystal orientation, possibly different from other grains

nitride

A compound with nitrogen

physical vapor deposition

Common technique used to synthesize thin solid films, see Chapter 5

\section{pseudopotential}

An effective potential that is used as an approximate description for complex systems

\section{Quantum-ESPRESSO}

Program used to run first-principles calculations

http://www.quantum-espresso.org

quarternary alloy

Alloy consisting of four elements

special quasi-random structure

Method for modeling random alloys in first-principles calculations, see Sec. 2.3.2 surface diffusion

The general process of adatom and admolecule motion on a surface

ternary alloys

Alloy consisting of three elements

transition state theory

Theory used to model diffusion, see Sec. 7.1

\section{Vienna ab-initio simulation package}

Program used to run first-principles calculations

https://www.vasp.at 


\section{Acronyms}

$\mathrm{B}_{k} \quad$ layered-hexagonal crystal structure

B1 rocksalt crystal structure

B3 zincblende crystal structure

B4 wurtzite crystal structure

CMD classical molecular dynamics

CVD chemical vapor deposition

DFT density functional theory

GGA generalized gradient approximation

HiPIMS high power impulse magnetron sputtering

KMC kinetic Monte Carlo

LDA local-density approximation

LSDA local spin-density approximation

MC Monte Carlo

MD molecular dynamics

MEMS microelectromechanical system

NEB nudged elastic band

PAW projector augmented wave

PBE-GGA Perdew, Burke, and Ernzerhof GGA

PVD physical vapor deposition

QE Quantum-ESPRESSO

QMC quantum Monte Carlo

QMD quantum molecular dynamics

SQS special quasi-random structure

TST transition state theory

VASP Vienna ab-initio simulation package 